\title{
TOWARDS ABSOLUTE DOSE MEASURMENT IN MRI-LINAC AND GAMMA KNIFE $\AA$ : DESIGN AND CONSTRUCTION OF AN MR-COMPATIBLE WATER CALORIMETER
}

\author{
By \\ Niloufar Entezari \\ Bachelor of Science with Honors \\ Physics \\ 2010 \\ Azad University of Urmia \\ Master of Science with Honors \\ Molecular and Atomic Physics \\ 2013 \\ Azad University of Mahabad
}

A thesis presented to Ryerson University in partial fulfillment of the requirements for the degree of Master of Science in the program of Biomedical Physics 


\section{Author's Declaration}

I hereby declare that I am the sole author of this thesis. This is a true copy of the thesis, including any required final revisions, as accepted by my examiners.

I authorize Ryerson University to lend this thesis to other institutions or individuals for the purpose of scholarly research.

I further authorize Ryerson University to reproduce this thesis by photocopying or by other means, in total or in part, at the request of other institutions or individuals for the purpose of scholarly research.

I understand that my thesis may be made electronically available to the public. 


\begin{abstract}
TOWARDS ABSOLUTE DOSE MEASURMENTS IN MRI-LINAC AND GAMMA

KNIFE®: DESIGN AND CONSTRUCTION OF AN MR-COMPATIBLE WATER

CALORIMETER

Niloufar Entezari

Master of Science, Medical Biophysics, Ryerson University, 2017
\end{abstract}

The purpose of this work was to design and build a portable $4^{\circ} \mathrm{C}$ stagnant Water Calorimeter (WC) for dual use in MRI-linac and Gamma Knife ${ }^{\circledR}$ in addition to conventional radiotherapy linacs. WC determines radiation energy absorbed in a sensitive volume absolutely and directly through measuring radiation-induced temperature rise (related to the medium's specific heat capacity). To assist with the design process, several parameters involved in calorimeter tank design including tank dimensions, a variety of insulation material and thicknesses, unique cooling design structures to sustain WC at $4^{0} \mathrm{C}$, as well as the calorimeter vessel design were simulated, and the results on heat gain/loss at the point of measurement was evaluated. Based on the optimizations, a calorimeter tank was built, and one single set of initial measurements in a conventional clinical linear accelerator was performed. 


\section{Acknowledgements}

This work has been made possible due to the contributions of many people. I would like to acknowledge a few of them here.

First, I would like to express my gratitude to my supervisors Dr. Arman Sarfehnia and Dr. Carl Kumaradas. Thank you for your patience and for always being willing to help me with whatever I needed. Your support and encouragement has made this work possible.

I would like to thank my committee members Dr. Claire McCann of Sunnybrook Health Sciences Center, and Dr. Vladislav Toronov of Ryerson University for their time and valuable feedback.

I would also like to thank to the engineers of the Medical physics department of the Odette Cancer Center of Sunnybrook Hospital, Gerard Peterson and Harry Easton, for constructing pieces of equipment and for supplying several tools used in this work.

Many thanks to Dr. Malcolm McEwen and David Marchington of NRC for their valuable participation in discussing the new design of a glass vessel for use in Gamma Knife ${ }^{\circledR}$ and constructing the first prototype of the spherical glass vessel.

I would like to acknowledge NSERC for providing funding for this work.

My sincere appreciation goes to all the staff, professors, and clinical physicists at the Ryerson medical physics unit for their help and support, as well as many thanks to all my friends, colleagues and fellow students. 
To my family 


\section{Contents}

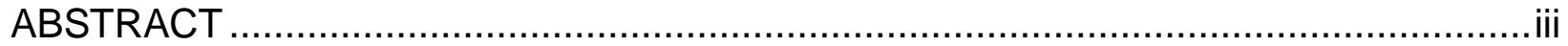

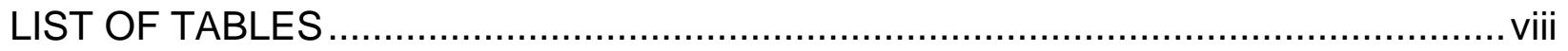

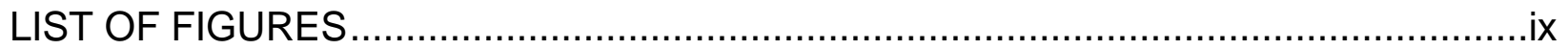

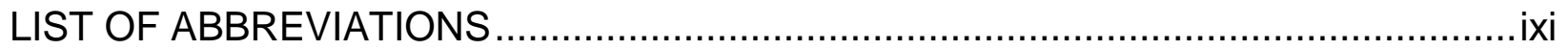

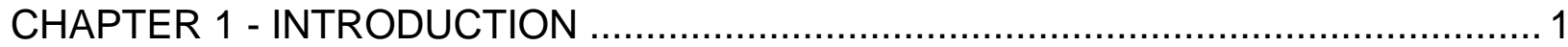

1.1 Cancer Prevalence and Its Treatment .......................................................... 1

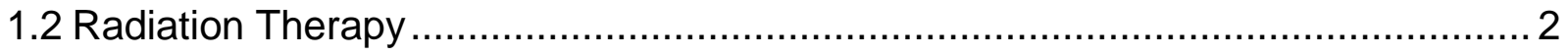

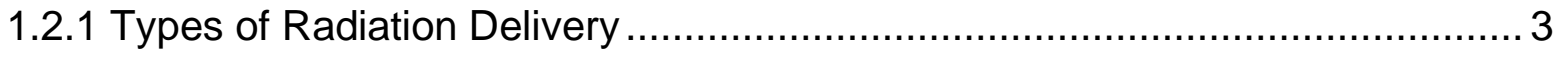

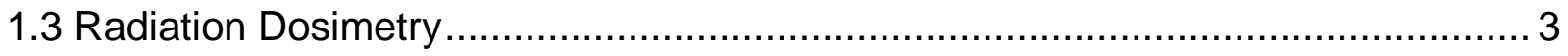

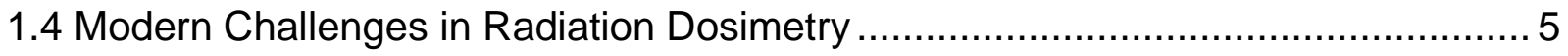

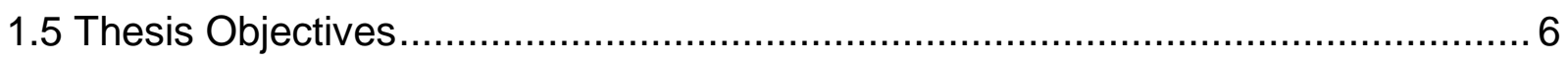

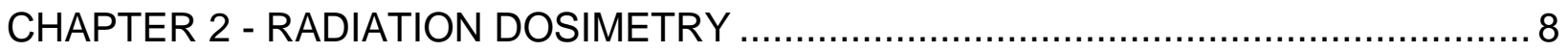

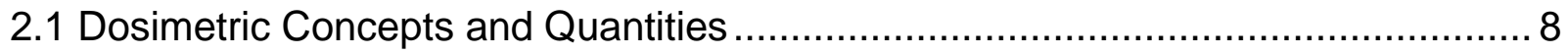

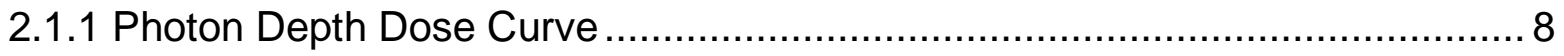

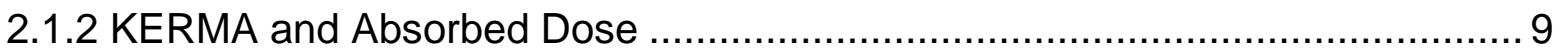

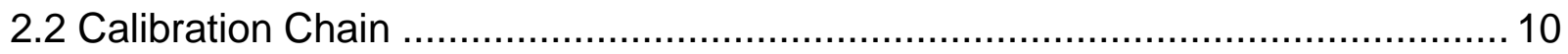

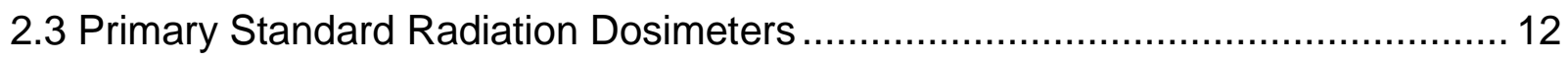

CHAPTER 3 - PRINCIPLES OF WATER CALORIMETER ..................................... 14

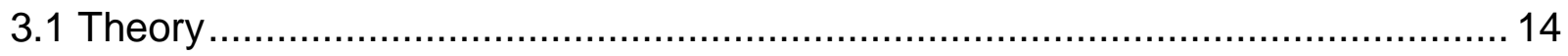

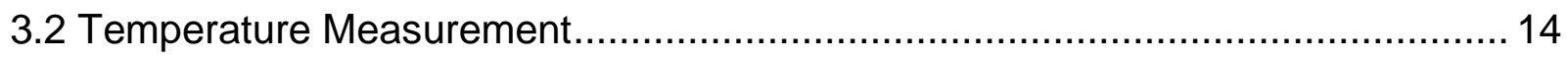

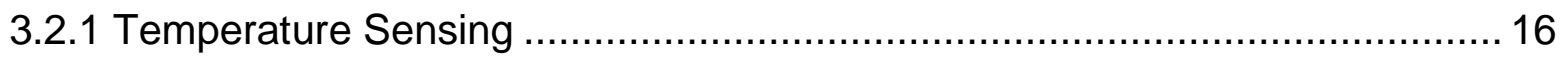

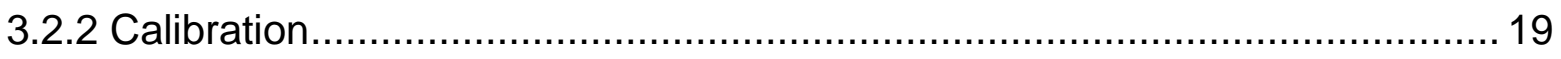

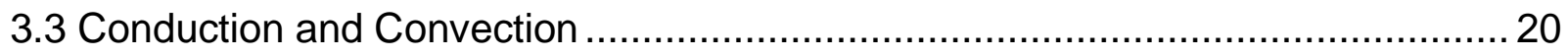

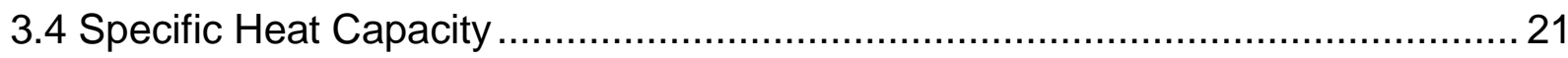

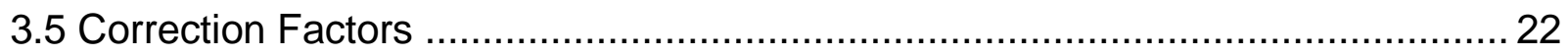

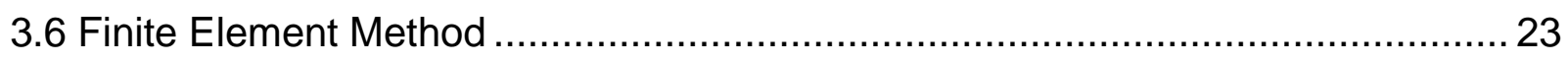

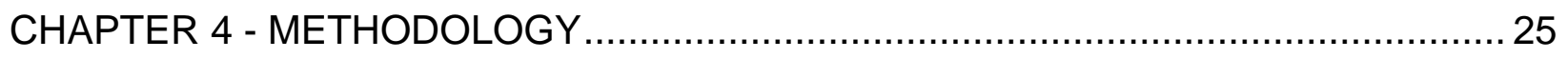

4.1 Water Calorimeter Design Selections ......................................................... 25

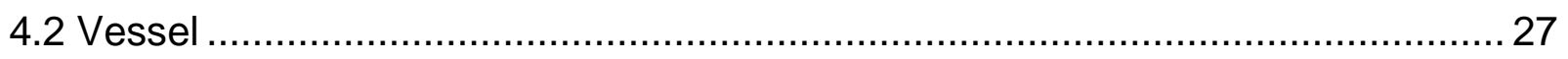

4.2.1 Parallel Plate Vessel (Plane Parallel Vessel) ............................................... 27

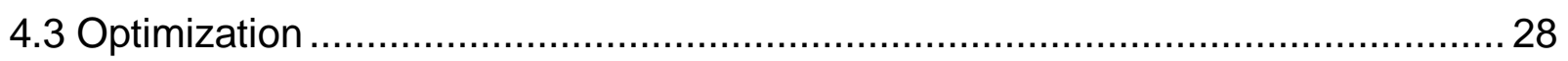

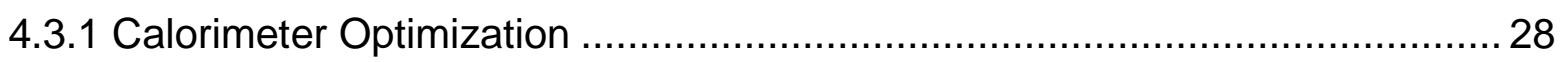

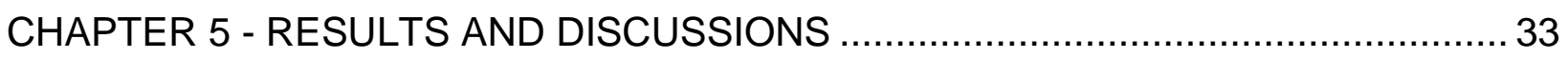




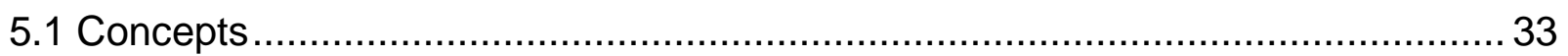

5.2 Study One: Calorimeter Design Optimization..................................................... 33

5.3 Study Two: Insulation Material Optimization .................................................. 38

5.4 Study Three: Plane Parallel Vessel Optimization ............................................... 39

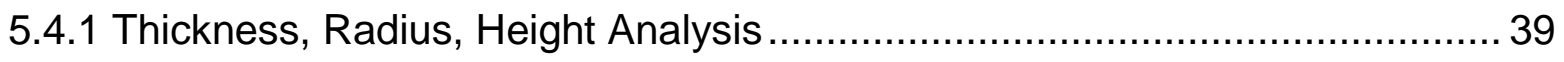

5.4.2 Thermistor Probes Position Sensitivity Analysis .......................................... 45

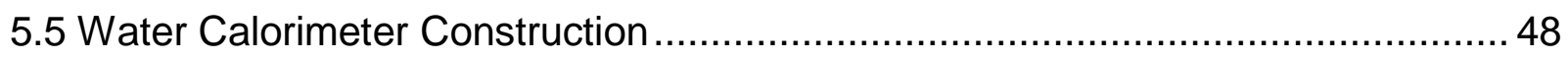

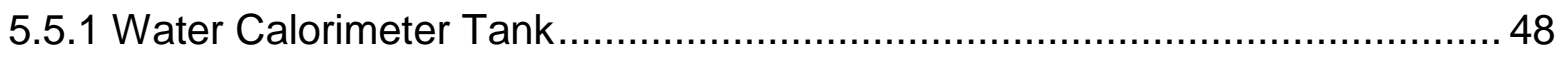

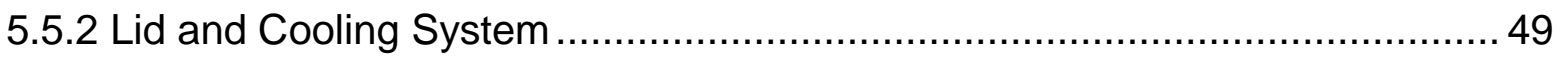

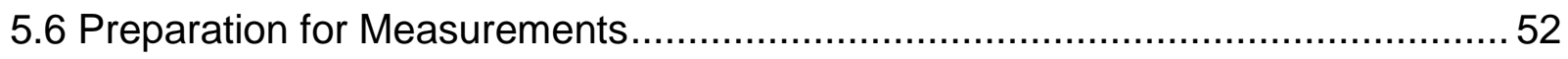

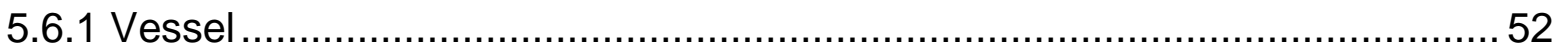

5.6.2 RTD and Thermistor Probe Calibration ....................................................... 54

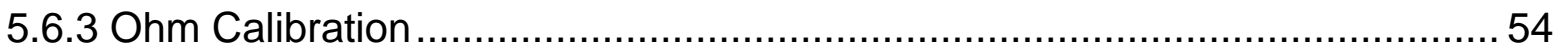

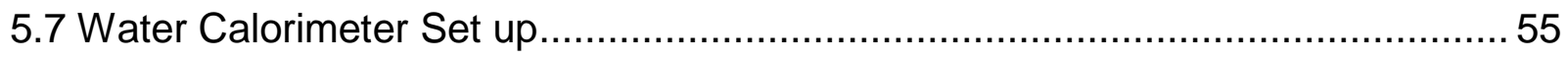

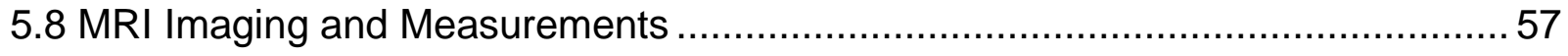

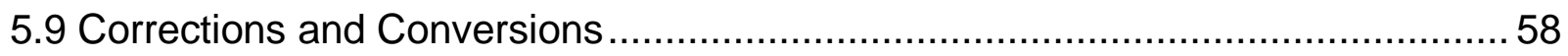

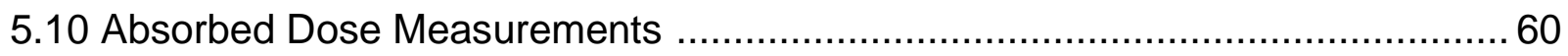

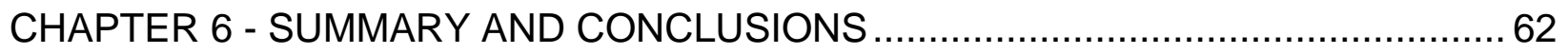

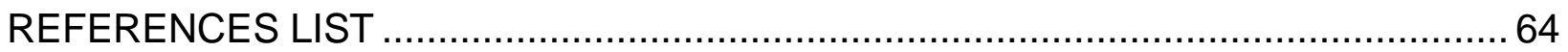




\section{LIST OF TABLES}

Table 5-1 Average $K_{\mathrm{ht}}$ of different thickness and different height for the radius of 39.5 $[\mathrm{mm}]$ of plane parallel glass vessel.

Table 5-2 Average $K_{\mathrm{ht}}$ of different radius and different height for the thickness of 0.5 [mm] of plane parallel glass vessel.

Table 5-3 Average percantage standard deviation of different thickness and different height for the radius of $39.5[\mathrm{~mm}]$ of plane parallel glass vessel.

Table 5-4 Average percantage standard deviation of different different radius and different height for the thickness of $0.5[\mathrm{~mm}]$ of plane parallel glass vessel.

Table 5-5 Average $K_{\mathrm{ht}}$ and percantage standard deviation of different points in plane parallel glass vessel from bottom to the top.

Table 5-6 Average $K_{\mathrm{ht}}$ and percantage standard deviation of different points in plane parallel glass vessel from right to the center. 47

Table 5-7 Sequences and slice thicknesses of MRI-imaging. .58

Table 5-8 Summary of dose measurments performed using the water calorimeter for 400 $\mathrm{MU} / 40$ s delivers under measurment conditions. 


\section{LIST OF FIGURES}

Figure 2-1 Absorbed dose and Kerma are shown as a function of depth in medium......10

Figure 2-2 A schematic description of the calibration chain ............................12

Figure 3-1 A typical water calorimeter measurment run showing the three stages of an experiment: pre-drift, irradiation, post-drift................................................ 16

Figure 3-2 A schematic circuit diagram of the whetstone bridge [13] ...................18

Figure 3-3 A schematic diagram of the full experimental setup..........................19

Figure 3-4 Steps involved in FEM analysis of the partial differential equations describing heat transport.

Figure 4-1 Evolution of water calorimeter designs: for (a) MRL, (b) Gamma Knife® and, (c) The final design .25

Figure 4-2 Rotation of water calorimeter tank in bores of (a) MRI-linac and (b) Gamma Knife $\circledast$. .27

Figure 4-3 A schematic picture of the glass vessel with two thermistore detectors positioned inside. .28

Figure 4-4 Several water calorimeter designs attempted in this work......................31

Figure 4-5 Glass vessel [13] 32

Figure 5-1 Results of different designs with different layers and size of air gaps for water calorimeter with constant temperature and water coolants at $4^{\circ} \mathrm{C}$ and ambient temperature of $22^{\circ} \mathrm{C}$

Figure 5-2 Results of different designs with different layers and size of air gaps for water calorimeter with increasing temperature and water coolants at $4^{\circ} \mathrm{C}$ and ambient temperature of $22^{\circ} \mathrm{C}$. 36

Figure 5-3 Long term drift curve simulations results based on the temperature fluctuations in water coolants for several water calorimeter designs. 37

Figure 5-4 (a) Different insulations attempted at thermal equilibrium of $4^{\circ} \mathrm{C}$ and the coolants also pass through the calorimeter at a constant $4^{\circ} \mathrm{C}$ with the ambient temperature of $22^{\circ} \mathrm{C}(\mathrm{b})$ The temperature in the coolants warms up. 39

Figure 5-5 Glass detection vessel, with the thermistor detectors aligned at the center. 40

Figure 5-6 Indicating the first and subsequent $k_{\mathrm{ht}}$ versus radiation runs 41 
Figure 5-7 (a) Heat transfer correction factor of optimization of the height and thickness of the plane parallel vessel at the fixed radius of $3.95[\mathrm{~cm}](\mathrm{b})$ Optimizing the height and radius of the plane parallel vessel at the fixed thickness of $0.5[\mathrm{~mm}]$.

Figure 5-8 (a) Standard deviation of optimization of the height and thickness of the plane parallel vessel at the fixed radius of $3.95[\mathrm{~cm}](\mathrm{b})$ Optimizing the height and radius of the

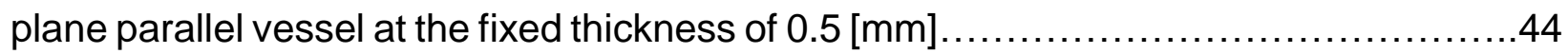

Figure 5-9 Different points from center to the right side of the glass vessel...............46

Figure 5-10 different points from bottom to the top of the glass vessel..................46

Figure 5-11 A schematic diagram of the water calorimeter positioned under a vertically incident radiation beam.

Figure 5-12 This figure indicates a CAD design of (a) The coolant lid (b) The detector holder and (c) Cross section of the in-house built water calorimeter.......................51

Figure 5-13 3-layer water calorimeter lid with stir puddles.............................52

Figure 5-14 Thermistore detectors are inserted through the ports on each side of the vessel. The vessel's small glass bulb is indicated in the red circle .......................53

Figure 5-15 Vessel bubbling process with pure Hydrogen...............................54

Figure 5-16 (a) Holder attached on the side of the tank (b) Leveling the glass vessel (c) Vessel and tank alignment (d) RTD probes secured to detector attachment

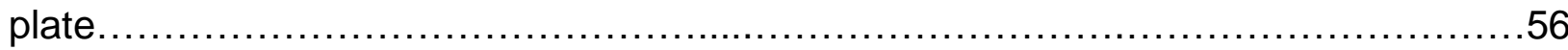

Figure 5-17 Three layer of lid assembly and wirings .................................56

Figure 5-18 Water calorimeter set-up (a) With all electronics and circuits (b) For applying measurements under linear accelerator.

Figure 5-19 The right image indicates the positioning of the water calorimeter inside the MRI. Image in the left indicates different sequences of the MRI images. .58 


\section{LIST OF ABBREVIATIONS}

2D Two dimensional

3D Three dimensional

CPU Central processing unit

${ }^{60} \mathrm{Co} / \mathrm{Co}-60$ Cobalt-60 radioisotope

AC Alternating current

AAPM American Association of Physicists in Medicine

ADCL Accredited dosimetry calibration laboratories

BIPM Bureau International des Poids et Mesures

CPE Charged particle equilibrium

$D_{\text {med }}$ Dose to medium

DNA Deoxyribonucleic acid

FEM Finite element method

GPIB General purpose interface bus

Gy Gray

IMRT Intensity modulated radiation therapy

$\mathrm{K}$ see KERMA

KERMA Kinetic energy released per unit mass

MC Monte Carlo

MU Monitor unit

MV/MeV Mega-electron volt

NIST National Institute of Standards and Technology

NPL National Physical Laboratory (UK)

NRC National Research Council of Canada

PDD Percent depth dose 
PDE Partial differential equation

PEEK Poly Ether Ether Ketone

PSDL Primary standards dosimetry laboratory

RTD Resistance temperature detector

SSD Source to surface distance

WC Water Calorimeter

$Z_{\max }$ Depth of maximum dose 


\section{CHAPTER 1 - INTRODUCTION}

\subsection{Cancer Prevalence and Its Treatment}

Cancer is the foremost cause of premature death, as measured by potential years of life lost. In 2016, it was estimated that in about forty percent of Canadians $(202,400)$ cancer would be developed in their lifetime, and one-quarter of Canadians $(78,800)$ were expected to die of the cancer disease [1].

Beyond this large impact, much progress has been made in the field of cancer detection and cancer therapy that has resulted in significant enhancements in early diagnosis of the disease as well as better treatment of cancer with the goal of controlling the disease while minimizing the damage to the healthy tissue.

Presently, there are five main types of cancer treatments [2]. The type of treatment patients receive will depend on the type of cancer that they have. The main types of treatment include surgery, chemotherapy, hormonal therapy, immunological therapy, and radiation therapy. Surgery plays an important role towards treating cancer by completely or partially removing the tumor. However, surgery may not always be possible given the spread of the disease, the accessibility to reach the tumour, or the location of the tumour. In many cases chemotherapy (the usage of drugs to attempt to kill the tumour or control the disease) is used as complementary treatment; however, almost all chemotherapy drugs result in some degree of toxicity to the patient. More often than not, chemotherapy will be combined with surgery radiation therapy (local treatment), and/or hormonal therapy. The goal of hormone therapy is to reduce the tumor in size until the patient is asymptomatic. Radiation therapy uses ionizing radiation to kill cancerous cells and shrink the tumours. Immunological methods of cancer treatments are designed to encourage the immune system of the body to attack the cancer cells in a way which does not hurt normal cells [2]. 
About half of all cancer patients receive radiation therapy as part of their treatment either as the sole mode of treatment or in combination with one of the other four treatment modalities. Radiation therapy can be delivered either externally (often with high energy particles) or internally (by placing a radioactive source inside the patient close to the tumour). Radiation therapy can use either directly ionizing radiation which includes charged particles such as electrons, protons, heavy charged particles, or indirectly ionizing radiation which includes neutral particles (such as photons and neutrons) to achieve its means. High energy external beam radiation therapy is the most commonly used form of radiation therapy in cancer treatment [2].

\subsection{Radiation Therapy}

The main objective of radiation therapy is maximizing radiation dose to malignant tissue and sparing healthy tissue. For this reason, the suitable type and number of radiation beams, the beam angles and energies of delivery are often carefully selected to achieve conformal treatment plans to the tumour while sparing surrounding critical normal structures. Physicians may also choose intensity modulation in order to increase the tumour control and decrease the normal tissue complication probability.

In radiation therapy, either directly or indirectly ionizing radiation are regularly used. Indirectly ionizing radiation leads to the interaction of uncharged particles (such as photons and neutrons) with the medium and generating of charged particles. Then energy is deposited in the absorber directly by these ejected charged particles. Besides, directly ionizing radiation (such as Electrons, protons, alpha particles, and other charged particles) are characterized as such since they deposit their energy in the absorber directly, causing excitations and ionization through direct Coulomb interactions.

lonizing radiation eventually leads to cell kill by damaging DNA via either direct or indirect interactions. When the radiation interacts directly with the DNA and its atoms in the cell, it may result in single- and double-strand breaks causing biological changes; This interaction may also happen indirectly when the charged particles interact with other 
atoms or molecules in the cell (particularly water which comprises about $80 \%$ of a cell) to produce free radicals (hydroxyl and hydrogen) that are able to then diffuse far enough to reach and damage DNA [3].

\subsubsection{Types of Radiation Delivery}

There are two means of delivering radiation to the intended region of interest $[2,4]: 1$ ) Brachytherapy which is the internal means of administering radiation by implanting or placing usually low energy/ relatively low activity radiation source(s) close to the tumour; 2) External beam radiotherapy takes advantage of relatively high energy radiation beams to administer the radiation dose to the patient from outside. Other than lower energy (orthovoltage) X-ray tubes, which have been around since the late 19th century, one of the first designs of a treatment delivery unit developed for external beam radiation therapy made use of Cobalt-60. In the Cobalt-60 teletherapy units, the radioactive isotope Co60 's high energy gamma ray photons emitted through nuclear decay processes are used to externally irradiate patients. Although the Co-60 teletherapy units are still in use today, they have been mostly superseded in developed countries by clinical linear accelerators (linacs). Both linear accelerators and x-ray tubes produce a bremsstrahlung photon spectrum by Coulomb interactions between accelerated electrons and the nuclei of high atomic number targets, what greatly distinguishes its application is the energy ranges involved. X-ray tubes produce kilovoltage energy range photon beams, and as such, are best suited for treatment of shallow and superficial tumours in comparison with linacs which produce megavoltage photon and electron beams and are suitable for deeper tumors. In linear accelerators, megavoltage electron beams can have a penetration of few centimeters, while high energy photons can deposit significant dose deep into tissue $[2,4]$.

\subsection{Radiation Dosimetry}

One major task in radiation therapy physics is to ensure that the planned and intended dose is accurately delivered to the patient. For this purpose, the physical quantity of radiation dose delivered to the toumor cell must be measured. 
Absorbed dose is a concept that can be applied to any form of ionization radiation, and is defined as the amount of energy absorbed in the medium per unit mass. The SI unit of dose is named gray[Gy], where $1[\mathrm{~Gy}]=1\left[\frac{\mathrm{J}}{\mathrm{Kg}}\right][4]$.

The total overall uncertainty on patient dose delivery is recommended to be known to within $5 \%[5,6]$. There are four major sources of uncertainty that contribute to the total dose uncertainty [6]:

I. Uncertainties on measurement of absorbed dose to water at a reference point under reference conditions.

II. Uncertainty on relative measurement of dose at non-reference distances and under non-reference conditions relative to the reference.

III. Uncertainty due to calculation of relative dose distributions by the treatment planning system (this includes uncertainty in image registration, tumour delineation, as well as 3D dose calculation algorithms).

IV. Uncertainty due to treatment setup and radiation dose delivery.

In this work, we focus on the first step (reference dosimetry).

As described above, clinically speaking, the first step towards proper dosimetry starts with a reference dose measurement that is used to establish the output of a particular radiation delivery unit in terms of dose per unit time or dose per monitor unit (MU) at a reference point under reference conditions. As will be discussed, this reference dose measurement is obtained in standard laboratories using highly specialized detectors. The second step towards determining the dose to a patient would be to understand/measure the relative dose at non-reference points and/or under non-reference conditions by relating a dosimeter reading to the clinical reference output. The reason for having separated the two techniques is because the detectors used for very accurate determination of output may not be appropriate for use under non-ideal and non-reference conditions. Conversely, the detectors that are sometimes used to measure the smallest of field sizes, etc. may not be appropriate for reference determination of absorbed dose. 
In order to measure the reference dose at a clinic, most commonly an air-filled ionization chamber is used as the detector of choice. The clinical ionization chambers are calibrated in terms of absorbed dose to water against an absolute dosimeter, such as a calorimeter, at a primary standard laboratory (PSL) under reference conditions. In the case of using the dosimeter in any beam quality other than the one in which it was calibrated, an absorbed dose beam quality conversion factor is used to convert the calibration factor from the reference beam quality to the specific beam quality in question.

The calibrated dosimeter can then be used for reference dosimetry in the clinics according to the established protocols [7].

As the body principally consists of water, the reference medium in radiation therapy dosimetry is taken to be water. As a result, dose to water is the quantity of interest in radiotherapy, and basic dose distribution data are typically measured inside a water tank (also known as a water phantom), or blocks of solid medium that have 'water-equivalent' properties including physical and electron densities, low atomic number elemental composition, etc. [3].

\subsection{Modern Challenges in Radiation Dosimetry}

As described previously, the main idea in clinical reference dosimetry is to establish the absorbed dose to water at the point of measurement directly and absolutely under reference conditions. The uncertainty in clinical dosimetry relies heavily on the uncertainty of the calibration factor provided by the standard laboratory as well as that of the beam quality conversion factor (taking the calibration factor from the reference conditions to the beam quality being measured). As such high caliber absolute absorbed dose measurement at the standard laboratories is of outmost importance for accurate clinical dosimetry.

However, although this technique was adequate for conventional radiotherapy units and high energy beams, with the advent of highly specialized clinical accelerators such as 
CyberKnife ${ }^{\circledR}$ and the integration of high field MRI with modern linear accelerators, or even GammaKnife ${ }^{\circledR}$, reference dosimetry is becoming ever more challenging. A large part of this is due to a deviation of treatment techniques and measurement conditions from the reference conditions defined in the current protocols.

One technique to accommodate absorbed dose measurements in such highly specialized units is to add corrections that account for non-conformities between the reference conditions (at the time of calibration) and measurement conditions (at user's clinic, at the time of clinical dosimetry). Alternatively, the primary standard techniques themselves could be modified to enable absolute dose measurements under conditions that more closely mimic those of the user. As an example, in the case of the integrated MRI-Linac (MRL), the current protocols may be adjusted to account for the non-compliant nature of these delivery techniques by addition of correction factors that account for not only the beam quality differences between the Co-60 and the MRL beam, but also for the effects of the magnetic field on the detectors. Such corrections will add uncertainty and may result in added complexity to the protocols as they will be dependent upon the strength of the magnetic field, the type of the detector, and even the orientation of the detector with respect to the magnetic field.

In this work, we have approached the problem by developing a water calorimeter that is designed for use not only in conventional linac beams, but also in an integrated high field MRI-high energy linear accelerators. Moreover, we have made the design such that the same calorimeter tank gives the possibility of doing future measurements under GammaKnife ${ }^{\circledR}$. This article focuses on describing the design of the calorimeter tank and the specifics of the optimization of the calorimeter tank and vessel.

\subsection{Thesis Objectives}

1. To deliver a detailed description of clinical dosimetry in radiotherapy, and comprehensive characteristics of the radiation dosimeter used in this work.

2. To numerically model and optimize a novel design for an in-house built water calorimeter, for use as an absolute clinical high-energy and portable dosimeter, by 
studying the effects of heat transfer (conduction) using the COMSOL Multiphysics ${ }^{\mathrm{TM}}$ (COMSOL Inc., v. 4.4) partial differential equation solver.

3. To construct a portable and MRI-compatible water calorimeter prototype based on the results from the numerical optimization stage.

4. To verify the feasibility of the water calorimeter as an accurate primary absolute dosimeter by measuring the absorbed dose to water in a 6MV high-energy clinical photon beam and comparing the results to the numerical results obtained in previous stages. 


\section{CHAPTER 2 - RADIATION DOSIMETRY}

\subsection{Dosimetric Concepts and Quantities}

This chapter briefly describes and defines the basic quantities and units of radiation dosimetry related to the body of work. The concept of dosimetry describes the measurement of the absorbed dose delivered by ionizing radiation.

As this thesis is based on development of a novel high-energy photon and electron dosimeter, and to show the feasibility of performing absolute dosimetry under a few highly specialized delivery equipments, an extensive portion of this chapter is dedicated to explain the theory of measuring dose, and the characteristics and units of dose used in this work.

\subsubsection{Photon Depth Dose Curve}

Percentage depth dose (PDD) is defined as the ratio of absorbed dose in a medium at any depth, to the absorbed dose at the reference depth (given identical source to surface distance) which is normally taken to be the depth of maximum dose, $Z_{\max }=Z_{\text {ref }}[4,9]$. Given photons of energy $h v$ traversing the absorbing medium, they will set electrons into motion. As it is indicated in figure 2-1, at depths shallower than the depth of maximum dose, there is a build-up region, whose thickness depends on the energy of incoming photons: A higher energy beam penetrates more, and has a deeper $Z_{\text {max }}$. The PDD can be divided into three regions: The build- up region (as shown in figure 2-1) is identified with a gradual increase in dose which is a consequence of the increasing number of primary/secondary electrons set in motion as the photon beam traverses and interacts with the medium. The dose increases up to the point of maximum dose at which point the number and energy of electrons entering a small volume of interest is equal to the number and energy of electrons leaving it (also known as charged particle equilibrium) [4,9]. For depths past $Z_{\max }$, the dose curve drops slowly as the attenuation of the primary beam overtakes the contribution to dose from charged particles created upstream and contributing to the point of interest (figure 2-1) [4,9]. 


\subsubsection{KERMA and Absorbed Dose}

KERMA (Kinetic Energy Released per unit MAss), is a measure of the amount of energy transferred at a point from indirectly ionizing radiation (such as photons and neutrons) to directly ionizing radiation (such as electrons) without taking into account any subsequent interactions and energy transfers [8]. Its unit is joule per kilogram $\left[\frac{\mathrm{J}}{\mathrm{kg}}\right]$, or gray [Gy] where $1[\mathrm{~Gy}]=1\left[\frac{\mathrm{J}}{\mathrm{Kg}}\right]$. Generally, for monoenergetic photons, Kerma is associated with the energy fluence, $\Psi(\mathrm{E})$, as indicated in the equation below:

$$
K=\Psi(\mathrm{E})\left(\frac{\mu_{\mathrm{tr}}}{\rho}\right) \quad\left[\frac{J}{K g}\right]
$$

$\frac{\mu_{\mathrm{tr}}}{\rho}$ is the mass energy transfer coefficient, which is the probability of the mean energy transferred by indirectly ionization radiation to the directly ionizing radiation while traversing the absorber.

The mass energy absorption coefficient, $\frac{\mu_{e n}}{\rho}$, quantifies the fraction of the energy absorbed in the medium along the path of particles. The mass energy transfer and absorption coefficients are related by [9]:

$$
\frac{\mu_{e \mathrm{n}}}{\rho}=\frac{\mu_{\mathrm{tr}}}{\rho} \cdot(1-\bar{g}) \quad\left[\frac{\mathrm{cm}^{2}}{\mathrm{~g}}\right]
$$

In quantifying the energy absorbed by the medium per unit mass, the mean value of energy which is transferred to charged particles and then lost through radiative process (bremsstrahlung and electron-positron annihilation) is denoted by a factor specified as the radiation fraction $\bar{g}$.

Absorbed dose, $D$, is the amount of energy lost through collisional interactions along the track of primary and secondary particles within a finite volume $\mathrm{d} V$. As electrons are 
traversing in the medium they their deposit energy along their tracks. The absorption of energy does not necessarily take place at the same location as the transfer of energy described by Kerma; however, not all the energy transferred from charged particles is absorbed in the medium, therefore absorbed dose is Kerma minus the energy carried away through radiation losses. Absorbed dose is also measured with the same unit as Kerma, joule per kilogram $\left[\frac{\mathrm{J}}{\mathrm{Kg}}\right]$ or gray [Gy]. The relationship between absorbed dose and Kerma for a typical photon beam, is illustrated in figure 2-1 [4,9].

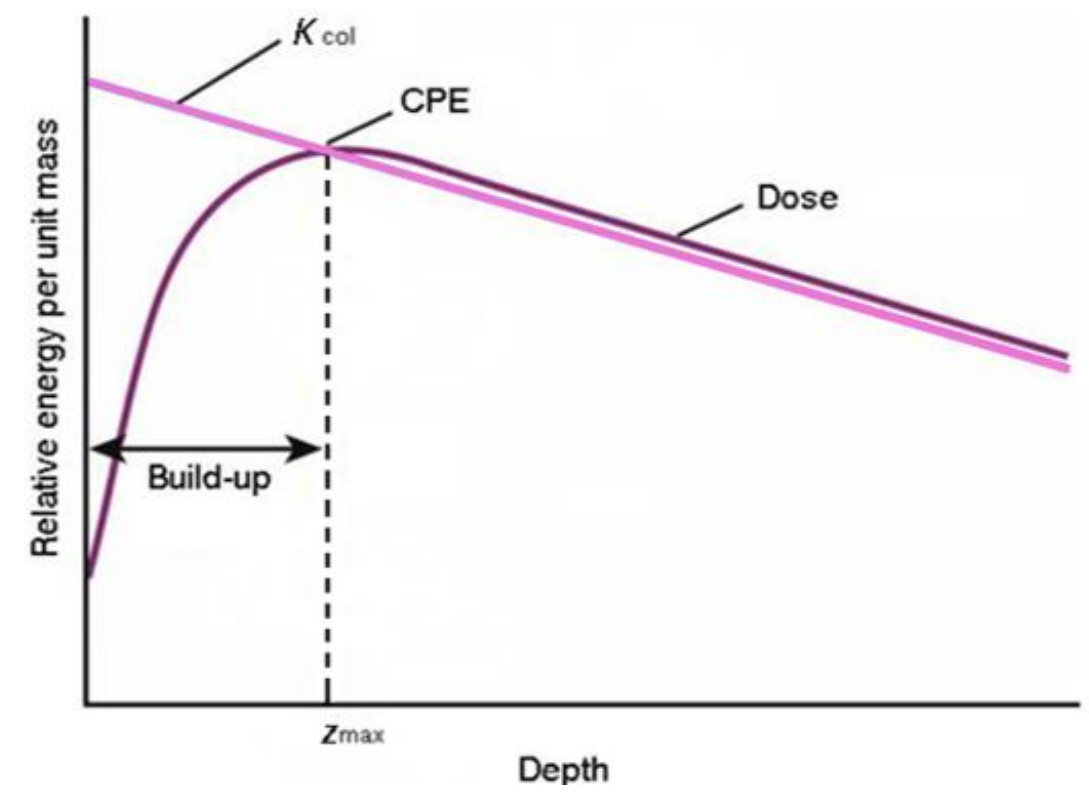

Figure 2-1 ABSORBED DOSE AND KERMA ARE SHOWN AS A FUNCTION OF DEPTH IN MEDIUM, WHERE Z $=0$ IS THE SURFACE OF THE MEDIUM (FIGURE ADAPTED FROM KHAN (2003)) [9].

\subsection{Calibration Chain}

The application of quality control causes the improvement of science and technology. A traceable standard and uniformity between definitions across different centers, cities, and countries allows for evaluation and comparison of results and improvement of outcomes.

None of the radiation detectors used in clinical setting are absolute dose detectors, meaning that none can measure dose directly and absolutely. They all act as substitutes, measuring quantities that relate, although not always directly, to radiation dose absorbed. 
Indeed, all currently available everyday clinical dose detectors require calibration. Calibration is simply the relation between absorbed dose to the detector and the corresponding measured signal. It is evident that the first requirement for successful calibration is availability of a known radiation source, the absorbed dose from which at a point under a set of reference conditions is well understood. Such primary standards are maintained at national and/or international dosimetry laboratories using special dosimeters that do not require calibration (more discussed in the next section). All other dosimeters used clinically are directly or indirectly traceable to such standard labs.

To this end, there are several stages of metrological organizations schematically shown in figure 2-2. Primary Standard Dosimetry Laboratory (PSDL) refers to a country's main national organization assigned to maintaining the country's radiation dosimetry standard [10]. BIPM (Bureau International des Poids et Measures) is an international organization that compares the standards and consistency between various Primary Standard Dosimetry Laboratories (PSDLs) around the world [11]. Both BIPM and PSDL use primary dose detectors and methods to determine absolute absorbed dose accurately and directly.

Primary Standards represent the unit for quantity, therefore PSDL and BIPM (in countries without PSDL) are not used for routine calibrations. In turn SSDLs are used for calibrating the detectors of the users for hospitals, cancer centers, and other laboratories across the countries based on their established standards $[10,11]$.

The nation's PSDL in Canada is named the Ionization Radiation Standards group of the National Research Council of Canada (NRC), which is the only organization maintaining air KERMA and absorbed dose standards and cross-calibrating users' secondary standards (NRC acts as Canada's PSDL).

The calibration factor of the user's dose detector (due to its robustness and high sensitivity) is traceable to one of these standard dosimetry labs mentioned above. The shorter the tractability of the detector, the fewer the number of cross-calibrations, therefore the smaller the overall uncertainty on the results. 


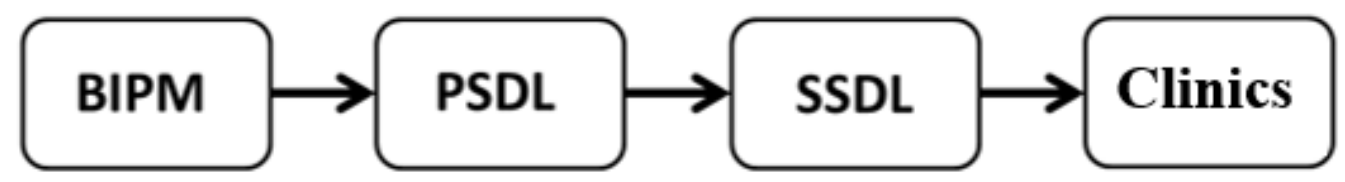

FIGURE 2-2 A SCHEMATIC DESCRIPTION OF THE CALIBRATION CHAIN $[10,11]$.

\subsection{Primary Standard Radiation Dosimeters}

Currently, three basic methods exist for measuring the absorbed dose to water at primary standard level:

I. lonometric method- In this method, a graphite cavity ionization chamber with a known volume and filled with air is used as a close approximation to a Bragg-Gray cavity theory in a water medium at a reference depth.

II. Fricke dosimetry- In chemical dosimetry standards, the dose is measured by the chemical change produced in the sensitive volume of the dosimeter due to the radiation. Irradiation oxidizes the ferrous ions $\mathrm{Fe}^{2+}$ in Fricke solution (FeSo4) into ferric ions $\mathrm{Fe}^{3+}$. Ferric ions show a strong absorption peak at a wavelength of $304[\mathrm{~nm}]$ which does not happen in ferrous ions.

III. Calorimetry- It is the most fundamental method in measuring the absorbed dose. It measures dose absolutely and directly inside the medium by measuring the radiationinduced temperature change at a point. Unlike all other primary standards, calorimetry is energy independent and depends on the specific heat capacity of the medium for conversion of its signal (temperature rise) to absorbed dose. Given that specific heat capacity of a material can be measured using other means than a known radiation source (including electrical means), a calorimeter can be self-calibrated in the absence of a known radiation source. There are two different methods in calorimetry, graphite and water calorimetry.

In graphite calorimetry, the smaller specific heat capacity of graphite relative to that of water results in larger temperature rises during irradiation than is the case of water calorimetry (hence, higher signal to noise ratio) $[4,9]$. 
However, the much larger thermal diffusivity of graphite causes rapid heat dissipation within the solid meaning that in graphite calorimetry the doses measured are only average doses inside a volume.

The principles of water calorimetry (and the main aim of this project) are described in detail in Chapter 3.

Except calorimetry, all other radiation detectors used in dosimetry measure a quantity other than dose, but one that is proportional or relates to dose. The factor describing the relationship is often termed the calibration factor. For example, the measured dose $D_{\text {det }}$ with cavity detectors (ionization chambers) used in clinical reference dosimetry should be converted to dose to the water $D_{\text {med }}$.

The remaining chapters of this work will focus on water calorimetry. The principles of water calorimeter operation, and the associated correction factors will be covered in detail in Chapter 3. 


\section{CHAPTER 3 - PRINCIPLES OF WATER CALORIMETER}

\subsection{Theory}

Calorimetry is one of the most fundamental and precise of all absolute dosimetry standards. Water calorimetry is a technique to measure the amount of energy absorbed in the water per unit mass in terms of temperature rise [12].

Water calorimetry measures the dose to water $D_{\mathrm{w}}$ based on its ultimate definition depending on temperature rise at a point as illustrated in following equations [12].

$$
\begin{gathered}
D_{\mathrm{w}}=\Delta \mathrm{T} \cdot c_{\mathrm{w}, \mathrm{p}} \cdot f_{\mathrm{w}}^{\mathrm{w}} \cdot \prod_{\mathrm{i}} k_{\mathrm{i}} \\
D_{\mathrm{w}}=\Delta \mathrm{T} \cdot c_{\mathrm{w}, \mathrm{p}} \cdot k_{\mathrm{ht}} \cdot k_{\mathrm{hd}} \cdot k_{\mathrm{p}}
\end{gathered}
$$

$\Delta \mathrm{T}$ is the radiation induced temperature rise at the point of measurement due to incident radiation.

$c_{\mathrm{w}, \mathrm{p}}$ accounts for specific heat capacity of water at constant pressure, $f_{\mathrm{w}}^{\mathrm{w}}$ refers to conversion from dose to detector to the medium (in water calorimetry, this conversion is unity as medium is water), and $\prod_{\mathrm{i}} k_{\mathrm{i}}$ accounts for correction factors that correct for the non-ideal measurement conditions. As shown in equation 3.2, $k_{\mathrm{i}}$ can be expanded onto $k_{\mathrm{ht}}$ (heat transfers correction factor), $k_{\mathrm{hd}}$ (heat defect correction factor), and $k_{\mathrm{p}}$ (perturbation correction factor) [15].

This chapter will focus on describing the definition and the principles behind water calorimetry and details of different variables of equation 3.2.

\subsection{Temperature Measurement}

In figure 3-1 a typical water calorimeter measurement run (also known as heat drift curve) is indicated. The heat drift curve is composed of three parts: The pre-drift, the irradiation 
period, and the post-drift. Ideally, in the absence of heat drifts, the pre-drift and post-drift would both be zero, as they represent a case without any radiation Induced temperature rise.

I- Pre-drift- This is the heat drift prior to the irradiation period measured by the detectors. If the water calorimeter was at perfect equilibrium conditions, then the pre-drift would have a slope of zero. However, more often than not, slight drifts present in the calorimeter may results in linear drifts which can to a large part be accounted for using extrapolation techniques which will be discussed below.

II- Irradiation period- During this interval, the temperature rises due to irradiation at a point measured by detectors (thermistor probes).

III- Post-drift- This is the temperature drift measured by thermistor probes after the end of the irradiation. In an ideal case without conduction and convection, the slope of the post-drift is zero. However, in reality a non-uniform dose distribution inside the water forms temperature gradients which may result in conduction and convection [13].

In a caloric run, temperature rise is measured by taking the difference between linear extrapolations of the pre-drift and post-drift to the mid-run (as shown in figure 3-1). In doing so, the effects of linear drifts can be compensated for through the extrapolation mechanism given the assumption that the drift continues to be present at a similar pace throughout the run.

It is common to take an extrapolation period for pre-drift and post-drift that are at minimum equal to the length of the irradiation. However, in the absence of strong non-linear drifts, $\Delta \mathrm{T}$ results are relatively insensitive to the pre-drift and post-drift intervals [13]. 


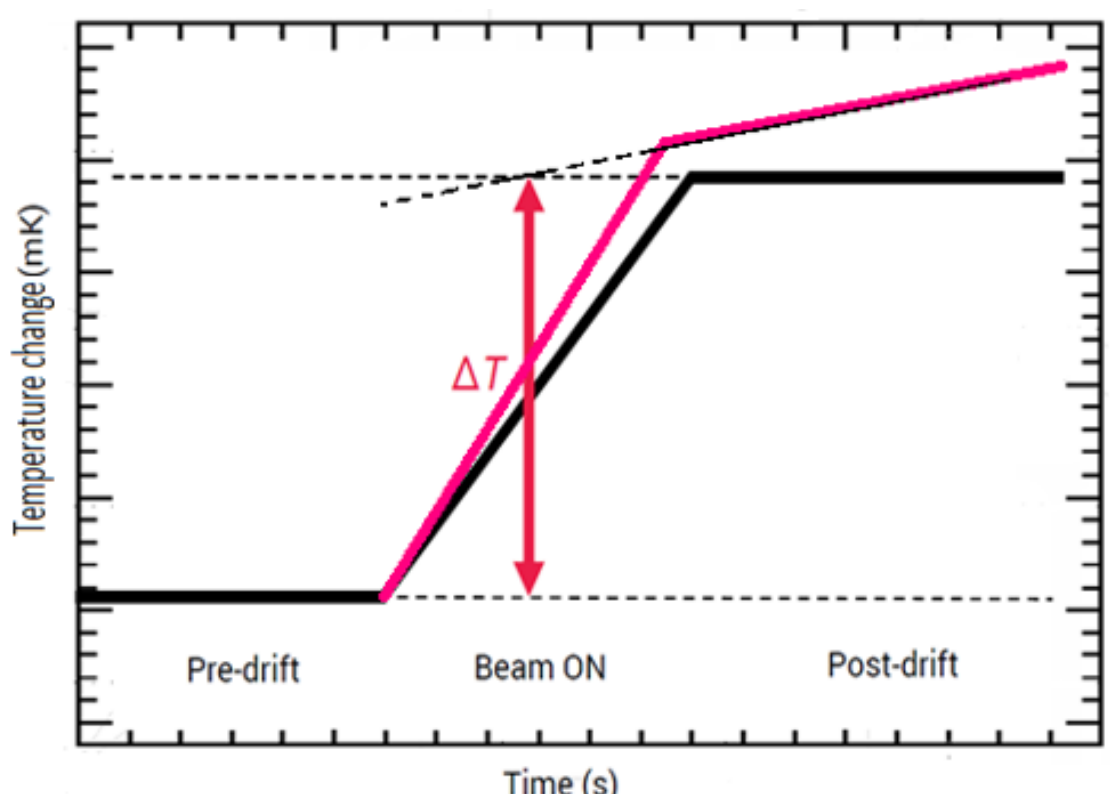

FIGURE 3-1 A TYPICAL WATER CALORIMETER MEASUREMENT RUN SHOWING ITS THREE STAGES PRE-DRIFT, IRRADIATION, POST-DRIFT [13]. THE DRIFT SHOWN IN BLACK CORRESPONDS TO AN IDEAL RUN (IN THE ABSENCE OF HEAT DRIFT), WHEREAS THE RED LINE IS MORE INDICATIVE OF A TYPICAL RUN IN PRESENCE OF HEAT DRIFTS.

\subsubsection{Temperature Sensing}

In this section, the equipment and circuitry used to measure the relatively small resistance changes in sensitive thermistor resistors will be explained. Figure 3-3 indicates a schematic diagram of all equipment setup and electrical connections used in this work which is at the heart of water calorimetry. In water calorimeter, the temperature is controlled and cooled to $4^{\circ} \mathrm{C}$ to reduce convection, since water has its highest density and smallest volumetric expansion at this temperature. By controlling the temperature of the entire water phantom at around $4^{\circ} \mathrm{C}$, the sub-millikelvin temperature rise due to radiation can be measured by means of thermistor probes.

To this end, two PT-100 RTD temperature probes are used to monitor the temperature of the water phantom. The RTD probes have a nominal resistance of $100[\Omega]$ at reference temperature $0{ }^{\circ} \mathrm{C}$. Reading temperature by means of RTD probes helps to adjust the temperature of the cooling fridge in order to prevent of the formation of temperature drifts 
in the water phantom. Resistances of these RTDs are measured by a Keithley K2000 digital multimeter. The RTDs and the digital multimeter are connected to a computer in the control room through a GPIB (General Purpose Interface Bus) extender. GPIB extender devices are used to transfer data over a coax cable between rooms. The extenders are used to maintain integrity of the signal while transferring data over large distances.

As indicated in figure 3-2, in this work a Wheatstone-type bridge, stated as an active bridge, is used to produce a signal in terms of voltage change.

A Wheatstone bridge contains two thermistors (with a total nominal resistance of $20[\mathrm{k} \Omega]$ at $4{ }^{\circ} \mathrm{C}$ ), and a decade resistor box connected in series in one arm. A decade resistor box is a resistor box where the resistance can be changed by the user. Two fixed 20 [k $\Omega$ ] resistors are serially connected to each other on the other arm of the bridge. The resistance decade box (burster) is adjusted to balance the resistance of the thermistors at the start of the experiment. When the resistance of the thermistors is balanced with the resistance decade box, the voltage reading between points $A$ and $B$ will be zero. When the temperature in the immediate surrounding of the thermistors changes (in our case due to irradiation of the calorimeter tank), the temperature-induced resistance change of the thermistors results in the bridge going out-of balance. As such, a small voltage proportional to the extent that the system is out of balance will be measured (and can be related back to resistance).

A reference AC signal oscillator, lock-in amplifier, is used for the voltage readout (as shown in figure 3-2). The lock-in amplifier measures the voltage difference between points $A$ and $B$ by inputting a signal (reference frequency) with an accuracy of few nano-volts. It provides the component with a reference internal sinusoidal frequency $\left(V_{\text {peak }}=1.5[\mathrm{~V}]\right.$, $f=1[\mathrm{~Hz}])$, and measures the output voltage while also detecting the phase and frequency of the output signal. The lock-in amplifier singles out only the frequency and phase component that does match the reference frequency (i.e. removes a large portion of random noise that would otherwise be out-of phase with the original input frequency). 


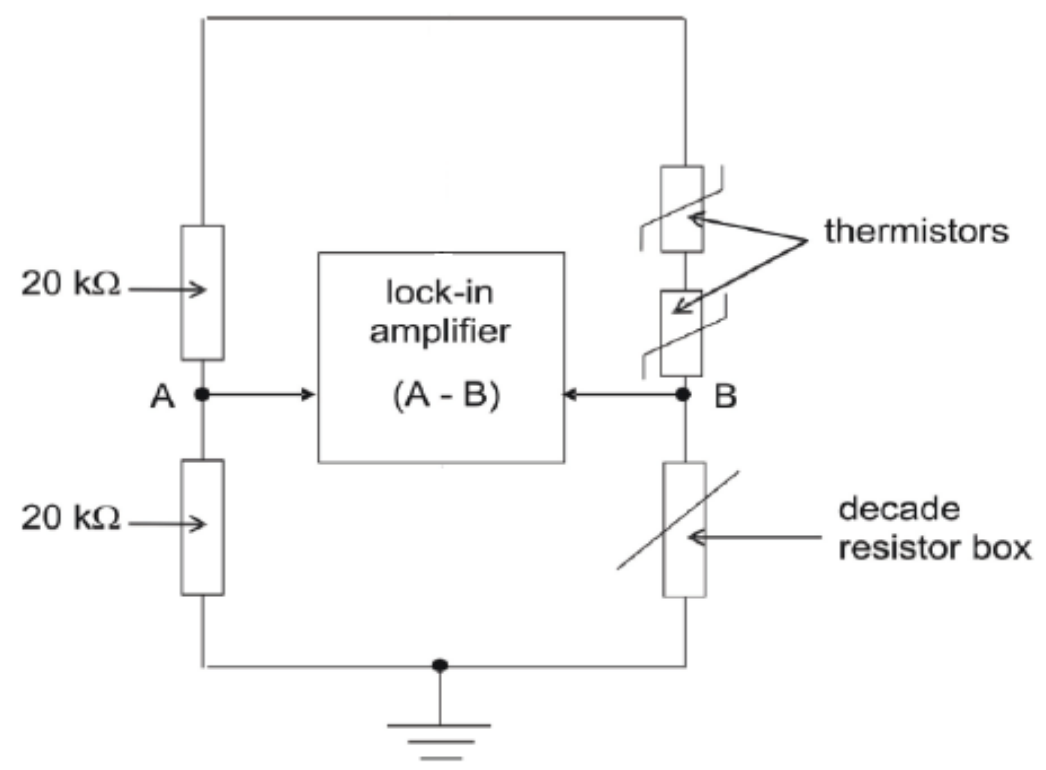

FiguRE 3-2 A SCHEMATIC CIRCUIT DIAGRAM OF THE WHEATSTONE BRIDGE [13].

Thermistors are temperature sensitive resistors that are used to measure small temperature rises accurately. Their small size and high temperature sensitivity makes thermistors perfect for use in calorimetry. Depending on whether the resistance increases or decreases with increasing temperature, thermistors can be categorized as either positive temperature change (PTC) or negative temperature change (NTC) $[4,9,13]$. A thermistor probe enables us to measure a temperature rise of few hundred micro-kelvin $[\mu \mathrm{K}]$ in water to a few mili-kelvin. 


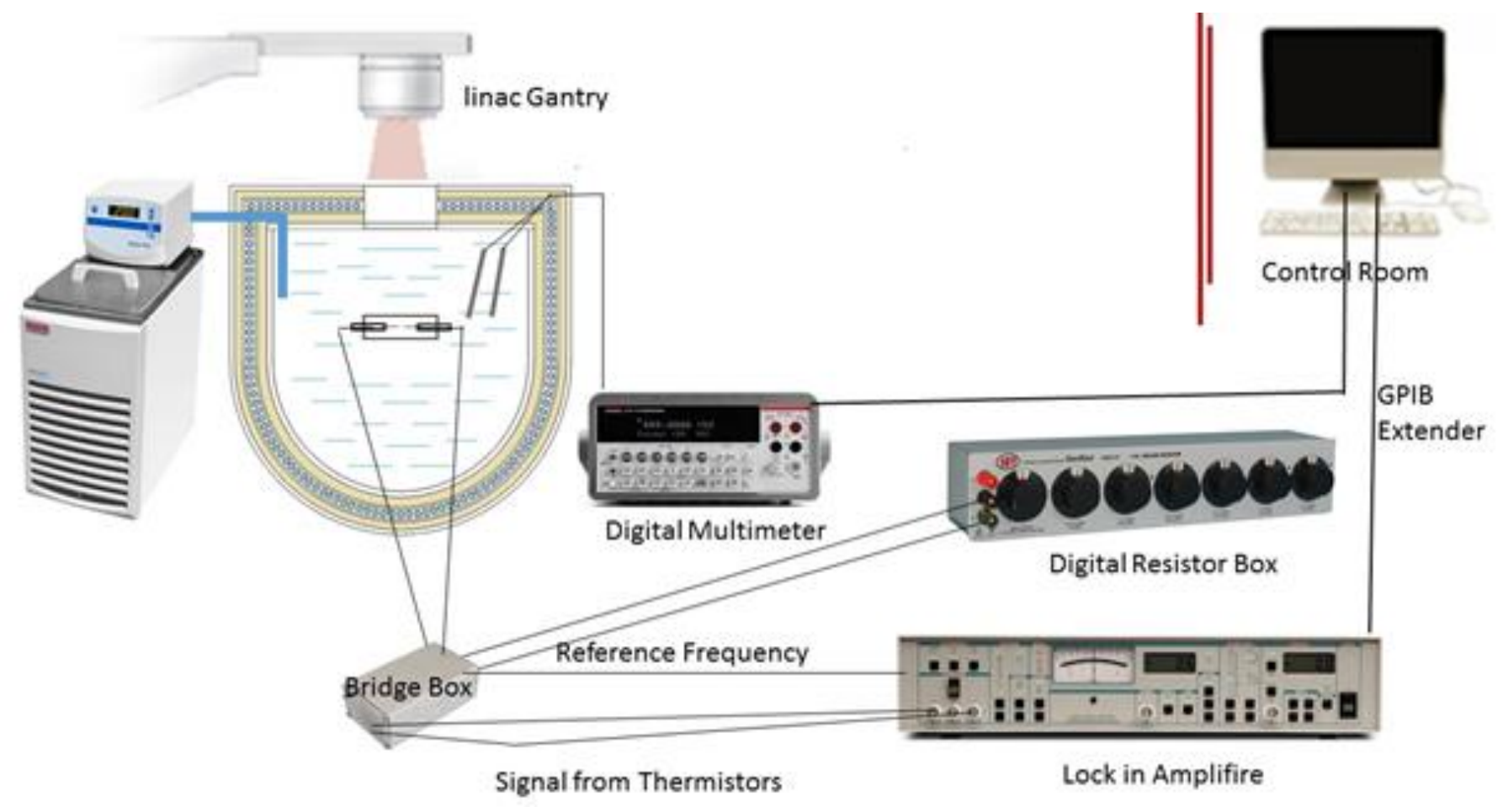

Figure 3-3 A SCHEMATIC DIAGRAM OF THE FULL EXPERIMENTAL SETUP. ALL COMPONENTS AND THEIR LOCATION INDICATED AS INSIDE TREATMENT ROOM OR CONTROL ROOM.

Thermistor beads are not located directly in the water; they are sealed in a Pyrex glass pipette. The pipette has $8[\mathrm{~mm}]$ diameter and $1[\mathrm{~mm}]$ wall thickness at one end while it changes to $0.6[\mathrm{~mm}]$ of outside diameter and $0.3[\mathrm{~mm}]$ of inside diameter at the very end of its tip.

\subsubsection{Calibration}

Water calorimetry is a primary standard as it can measure absorbed dose from an unknown radiation beam directly without being cross-calibrated against any known radiation source. As long as the temperature is identified, a calorimeter can measure dose. However, the temperature is measured from an output signal of the voltage as a result of a resistance change in thermistors.

Three steps of calibration are required for water calorimetry which are described below: 
The first step is RTD probes calibration against a thermometer. Second step is thermistor calibration against the calibrated RTD probes in the first step, and the third is bridge calibration. Bridge calibration is the relation between the responses of the active bridge in terms of change of voltage to a known change in thermistor resistance as a function of temperature change. It is known as the ohm calibration.

\subsubsection{RTD Probe Calibration}

For each RTD calibration, a 'resistance vs. temperature' calibration curve is established. The two RTD probes used in this work are cross calibrated against a calibrated thermometer traceable to NIST (National of Institute and Technology) which allows to read off the temperature of the coolant to better than $0.02{ }^{\circ} \mathrm{C}$.

The RTD probes are calibrated in a cooling fridge circulator (Neslab RTE-7). The calibration is performed at a temperature range of $-4^{\circ} \mathrm{C}$ to $12^{\circ} \mathrm{C}$. The resistance of RTD probes at each temperature has been read by the multimeter.

\subsubsection{Thermistor Probe Calibration}

The second step is calibrating thermistors against the calibrated RTD. The thermistors are calibrated over the same temperature range as were the RTDs in the controlled coolant. From this calibration step we obtain the temperature-resistance calibration of the thermistors.

\subsubsection{Ohm Calibration}

The third calibration curve in this work is called the bridge calibration or Ohm calibration. It is performed during the actual measurements. It is done by changing the resistance of the decade box (burster) by $1[\Omega]$ in the absence of large drifts during the experiments. As such, the voltage change as a function of one ohm resistance change for an array of water bath temperatures can be obtained and used as the calibration between voltage and resistance, completing the loop.

\subsection{Conduction and Convection}

There are three mechanisms of heat transfer through a medium. They are: conduction, convection and radiation. Conduction is the major process in calorimetry and effects due 
to convection can require correction in special cases. Radiation is always negligible since we are operating at low temperatures [14].

In this section, the bases for equations describing heat transport are presented. We can solve these heat transport equations for complicated systems such as a calorimeter by using finite element method (FEM) modeling and numerical analysis software packages. Conduction is a process of energy transfer through material of a medium either in a solid or liquid medium due to temperature gradients that form in the medium. The heat transfer rate per unit area, $\dot{q}$, is dependent upon the magnitude of the thermal gradient, $\nabla T$, and the thermal conductivity, $k$, of the medium:

$$
\overrightarrow{\dot{q}}=-k \vec{\nabla} T \quad\left[\frac{w}{m^{2}}\right]
$$

Convection, on the other hand, is the heat transfer in a gas or liquid by the circulation of currents from one region to another. It is governed by a heat transfer coefficient, $h$, and the temperature difference between the fluid, $T$, and the adjacent surface, $T_{S}$.

$$
\dot{q}=h\left(T_{s}-T\right) \quad\left[\frac{W}{m^{2}}\right]
$$

Based on the properties of water, at $3.98^{\circ} \mathrm{C}$ water has its highest density and the volumetric expansion coefficient of water is zero. As such by operating the water calorimeter in a tight temperature range around $4^{\circ} \mathrm{C}$ (more specifically $3.98^{\circ} \mathrm{C}$ ), effects of convective flow are minimized. Given our approach was to operate a $4^{\circ} \mathrm{C}$ cooled water calorimeter; it is a good approximation, to ignore the effects of convection during simulation for high energy photon beams.

\subsection{Specific Heat Capacity}

Specific heat capacity of water is temperature dependent. In water calorimetry, the increase of temperature gradients in water due to incident radiation beam is in the order of micro-kelvin; therefore, a constant value of $c_{w, p}$ at nominal calorimeter is used. In this 
work, we used a constant $c_{\mathrm{w}, \mathrm{p}}=4.205 \times 10^{3}[\mathrm{~J} / \mathrm{kg}$. K $]$ at $4^{\circ} \mathrm{C}$ calorimeter, since temperature gradients are small.

\subsection{Correction Factors}

The correction factors, $\prod_{i} k_{\mathrm{i}}$, in equation (3.2) involved in water calorimetry are Described below:

The heat transfer correction factor, $k_{\mathrm{ht}}$, is one of the most important correction factors in water calorimetry. It corresponds to the effects of heat transfer due to conduction (and possibly convection) of the temperature gradients inside the calorimeter.

$k_{\mathrm{ht}}$, is the ratio of the calculated ideal temperature rise in the absence of heat transfer, to the calculated realistic temperature rise at the point of measurement by taking into account heat transfer effects. The more accurately the model of the calorimeter and the understanding of the overall dose distribution inside the water tank, the more accurate the determination of $k_{\mathrm{ht}}$ (e.g. finite element method calculations was done to this end in this project). The modeling will be further discussed in following sections.

The ideal temperature rise can be calculated simply by setting the thermal conductivity of the absorbing materials to zero.

One of the principal disadvantages of water calorimetry is the heat defect associated with radiation induced chemical reactions with water impurities can either result in heat release or energy absorbance from the surrounding depending on the type of reaction (exothermic vs. endothermic) which we try to correct for using the $k_{\text {hd }}$ correction factor. As the purity of water and concentration of dissolved gases cannot be controlled in the huge volume of water inside the water tank, we encompass the thermistor probes inside a vessel filled and sealed with extra pure water $(<2 \mathrm{ppb})$. Just prior to sealing off the vessel, however, the extra pure water inside is bubbled with a known extra pure gas to remove any dissolved gases and saturate and replace the water with a known pure gas. 
For the purposes of this work, 99.999\% pure hydrogen gas was used. The heat defect for pure water saturated with hydrogen is known to be zero. Additionally, Pyrex glass is often used to construct the vessel as it is extremely unreactive. The presence of the glass vessel is one of the largest sources of radiation beam perturbation [15].

The $k_{\mathrm{p}}$ perturbation correction factor is the product of the radiation field that perturbation occurs due to the effects of non-water materials present in the water calorimeter [13].

\subsection{Finite Element Method}

To calculate the heat transfer correction factor, we used Comsol Multiphysics ${ }^{\mathrm{TM}}$ software in this work. Comsol Multiphysics ${ }^{\mathrm{TM}}$ is a Finite Element solver and a numerical technique to solve the time dependent Partial Differential Equations (PDE) for heat transfer and integral equations over a specified domain.

It works by discretizing the geometry and splitting it into a finite number of smaller elements which are called mesh (figure 3-2b). By breaking down the geometry to the optimal mesh element sizes, and solving the partial differential equations relating to the physics in every given mesh element by respecting the boundary conditions and continuity between mesh elements, the individual results are combined to form a union solution over the entire domain [16].

The modeling workflow in Comsol Multiphysics ${ }^{\mathrm{TM}}$ is, setting up the model environment which is heat transfer in this work. In modeling heat transfer, we need to define a geometrical representation of the physical problem and determine the properties of the materials for each part. Subsequently, boundary conditions and heat sources are defined and set. In our work, the energy absorbed from radiation dose incident on the calorimeter is one major source of heat. Finally, we can extrapolate the results in form of temperature rise during the time.

COMSOL Multiphysics ${ }^{\mathrm{TM}}$ allows for the definition of $2 \mathrm{D}$ axially symmetric geometries (mirror application). Using a symmetric model can lead to less intensive and faster 
computational simulations, since the number of degrees of freedom of the model directly affects the solution time. The shape of the water calorimeter itself naturally leads to $2 \mathrm{D}$ axially symmetric modeling, which helps in reducing the number of mesh elements required to describe the geometry.

The heat transport module in COMOSL Multiphysics ${ }^{\mathrm{TM}}$ environment that is used in this work was validated both in the presence and absence of convection by Sarfehnia (2010) [13]. In this work heat transfer due to conduction was studied and convection was fully ignored. This is based on the fact that the temperature gradients formed inside the water calorimeter following energy absorption from high energy photon beams are small (milliKelvin). Furthermore, operation of the calorimeter at a nominal $4^{\circ} \mathrm{C}$ ensures minimal convection at these small gradients because at $4{ }^{\circ} \mathrm{C}$ water has its highest density and lowest volumetric expansion coefficient.

(a)

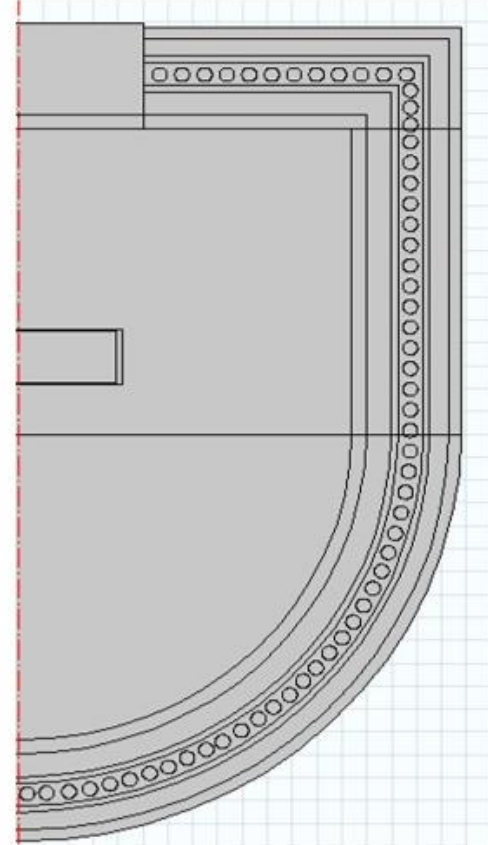

Geometry and Material (b)

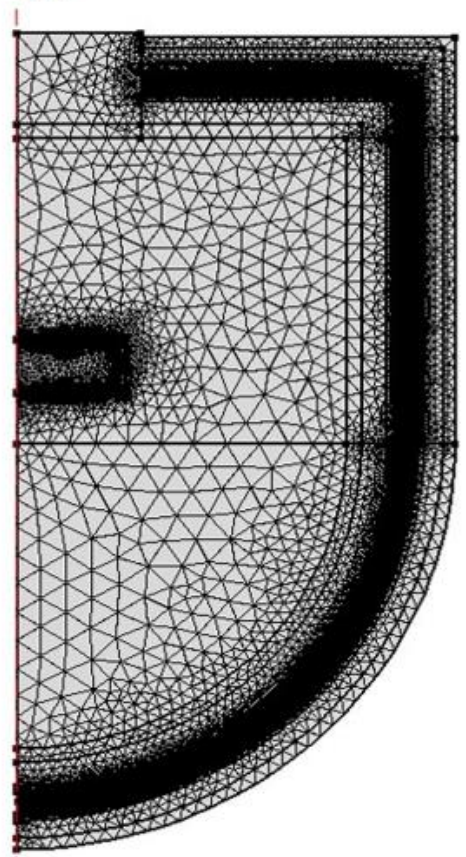

Mesh Discretization (c)

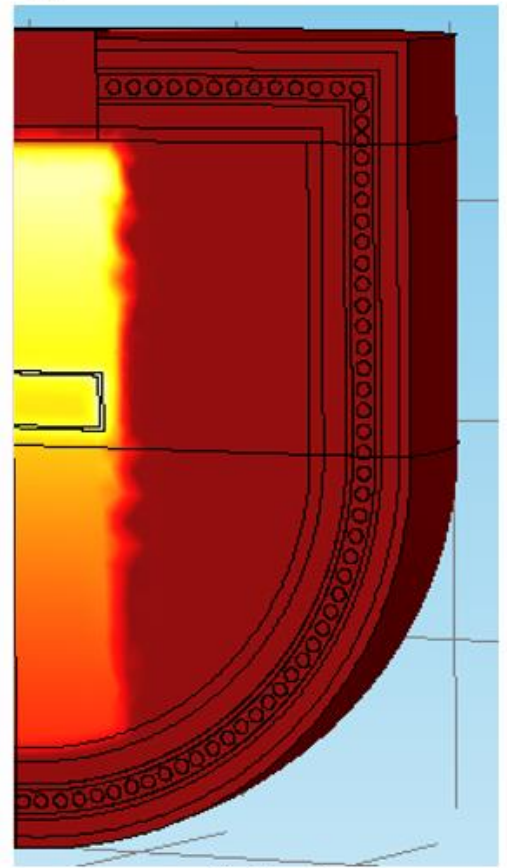

Solution

FIGURE 3-4 STEPS INVOLVED IN FEM ANALYSIS OF THE PARTIAL DIFFERENTIAL EQUATIONS DESCRIBING HEAT TRANSPORT: (A) A DEFINITION OF THE GEOMETRY, BOUNDARY CONDITIONS AND MATERIAL PROPERTIES, (B) A DISCRETIZING MESH USED BY THE SOLVER TO BREAK DOWN THE PROBLEM, AND (C) ANALYSIS OF RESULTING SPATIALLY-VARYING, TIME-DEPENDENT SOLUTION. 


\section{CHAPTER 4 - METHODOLOGY}

\subsection{Water Calorimeter Design Selections}

In this work, we used the numerical design optimization software Comsol Multiphysics ${ }^{\mathrm{TM}}$ to simulate and better understand our portable, and MRI compatible water calorimeter, and study its operational response under clinical high energy. We also calculated the heat transfer correction factor by using the Finite Element Method (FEM).

Our goal was to go beyond simply an MRI-safe design, but attempt to develop a calorimeter tank that is MRI-compatible, such that MRI imaging of the calorimeter tank for possible purposes of positioning can be performed without significant image perturbation. As such, every component of the calorimeter tank was designed from non-ferromagnetic components.

(a)

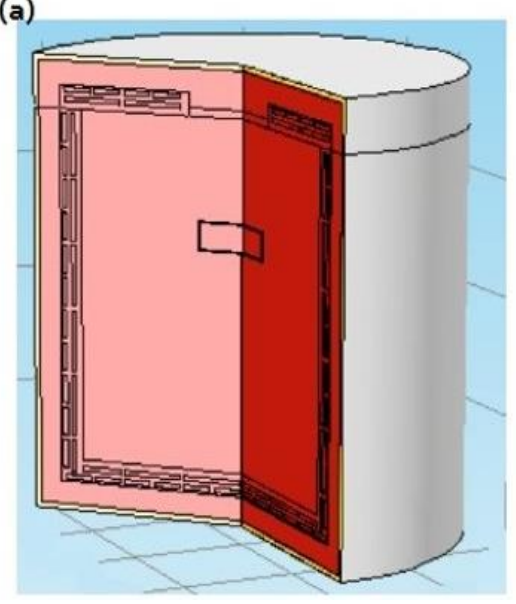

Figure 4-1 Evolution Of WATER CALORIMETER DESIGNS: FOR (A) MRI-LINAC, (B) GAMMA KNIFEß AND, (C) MRI-LINAC AND GAMMA KNIFEß.

(c) (b)

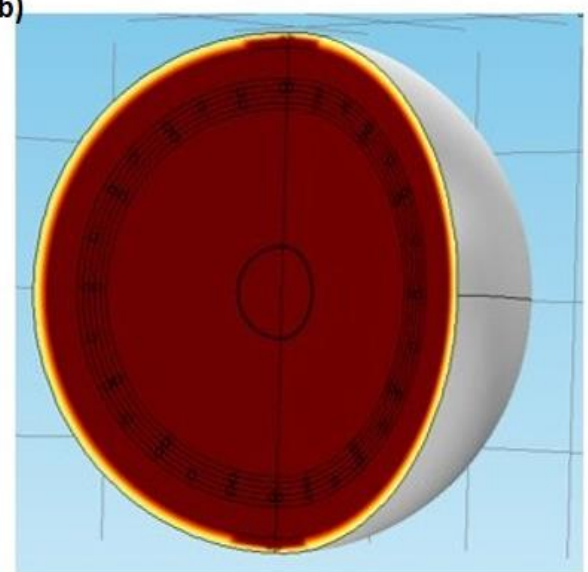

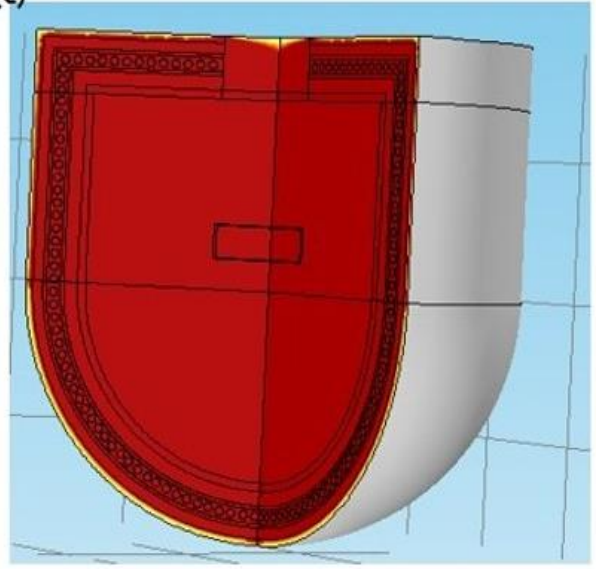

As indicated in figure 4-1 we considered three different designs. Initially we decided to design a cylindrical water calorimeter tank to operate it in the MRI-linac with the radiation beam incident vertically from the top (figure 4-1a), and another spherical water calorimeter that would best accommodate Gamma Knife® with the 192 sources focused at the center of a spherical phantom (figure 4-1b). The latter would ensure that every beam has an equal path length and as such contributes the same given dose. However, 
the final decision in this work was to combine the two designs to create a more versatile and economical design.

The final design of our in-house water calorimeter is a hybrid of two independent designs noted above (figure 4-1c). We selected the dimensions of the tank such that it fits inside both, the MRI-linac (MRL) and the Gamma Knife® as well as of course all conventional units. The tank materials were chosen such that the tank is fully MR-compatible. The tank is setup and used vertically (with the beam incident from the top) in MRL water calorimetry (figure 4-2a), and it is setup horizontally when used for Gamma-Knife® water calorimetry

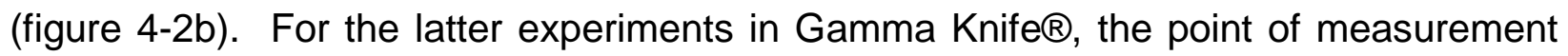
would be the center of the hemispherical portion of the phantom with all the 192 Co-60 sources focused on this center ensuring all the beams have identical path lengths.

The exact dimensions, details of the insulation and geometry, as well as the location and mechanism of cooling system (to bring down and maintain the calorimeter temperature at $4^{\circ} \mathrm{C}$ ) were determined based on a comprehensive set of simulations. The methodology for the simulations will be better described in section 4.3. It should be noted that commonly calorimeters are only designed and built by primary standard laboratories and their design generally is based on decades of experience. However, the goal here was to observe if optimization can provide an insight into superior design to traditional designs, given such a large deviation and leap of our general shape from conventional cubicshaped calorimeter tanks. 
(a)

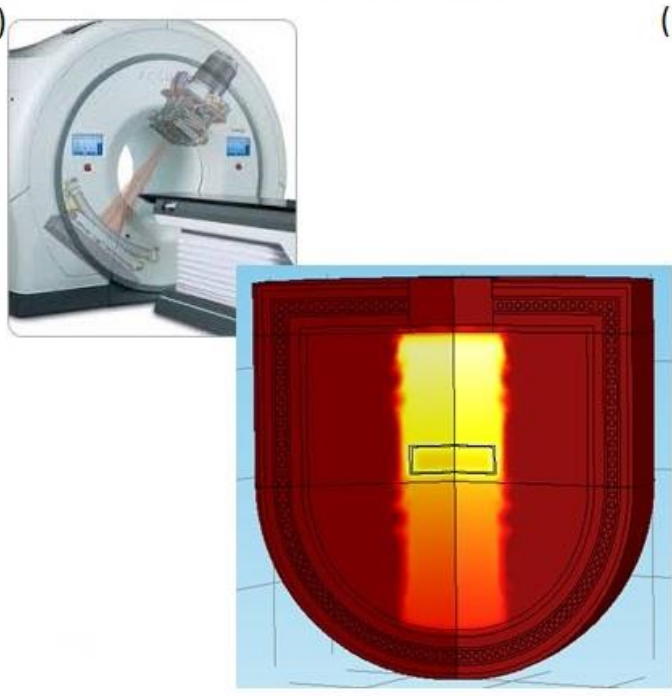

(b)

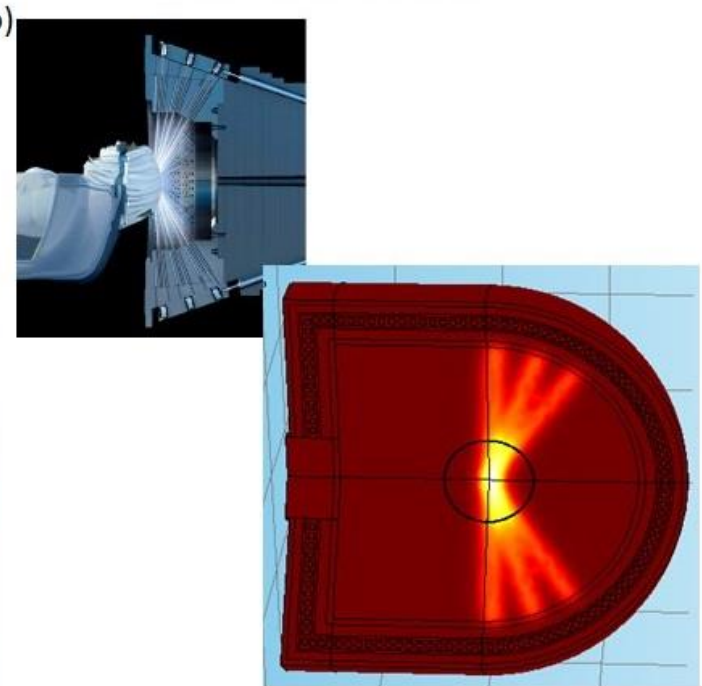

Figure 4-2 ROTATION OF WATER CALORIMETER TANK IN BORES OF (A) MRI-LINAC AND (B) GAMMA KNIFE®.

\subsection{Vessel}

\subsubsection{Parallel Plate Vessel (Plane Parallel Vessel)}

The glass vessels used in calorimetry conventionally have two generic shapes. The vessels can either be parallel plate or cylindrical. The experience of our research team with parallel plate vessels has been extremely positive, and as such we set out to use those in these set of experiments as well. Given our history using a parallel plate vessel in other calorimetric campaigns including in conventional high energy photon and electron beams [17], HDR-192 brachytherapy [18], proton and carbon ion dosimetry [19], we decided to use an identical design shape vessel also for use in our novel design calorimeter in MRI-linac.

A schematic diagram showing this vessel can be seen figure 4-3. The vessel has a front and back window thickness of 0.7 [mm] with an inner separation of $22.66[\mathrm{~mm}]$. Side glass thickness of this vessel is 1.96 [mm], and its radius is $39.5[\mathrm{~mm}]$. The vessel has a port in each side to insert the thermistor detectors through them. These ports are sealed with CAPFE $\AA$ Teflon encapsulated O-rings to seal the high pure water and keep it inside the 
vessel. The third port is attached on this vessel to fill and bubble the water. A small bulb on top of the third port is mounted to trap a gas bubble after saturation process [13]. This is to avoid breakage of the glass vessel as it is cooled down from room temperature to $4^{\circ} \mathrm{C}$. The glass vessel itself is mounted on a holder which itself is fixed to the inner wall of water calorimeter.

(a)

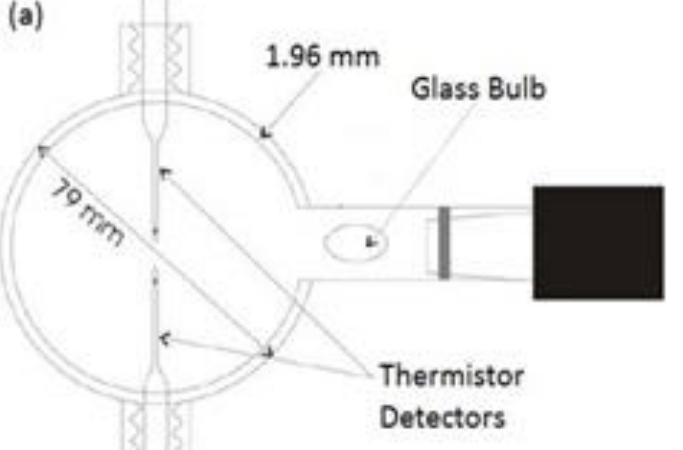

Detectors (b)

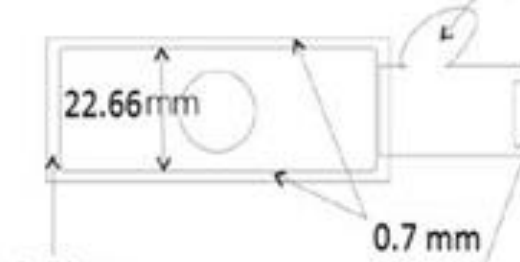

$1.96 \mathrm{~mm}$
Glass Bulb to Trap

Gas Buble

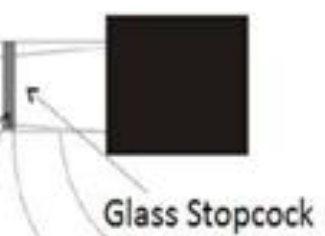

CAPFE Teflon

Coated O-ring

FIGURE 4-3 A SCHEMATIC PICTURE OF THE GLASS VESSEL WITH TWO THERMISTOR DETECTORS POSITIONED INSIDE (REPRODUCED FROM SARFEHNIA (2010) WITH PERMISSION) [13].

As part of our optimization work for the calorimeter tank, we decided to also try to explore if the vessel design can also be improved. Although calorimeter vessels are generally hand-made by a professional glass blower and the dimensions used are based on experience, in this work we made an attempt to see if simulation of the vessels with different dimensions and glass thicknesses can give us an insight into improved designs.

\subsection{Optimization}

\subsubsection{Calorimeter Optimization}

To build a calorimeter, several factors / parameters need to be considered:

I. Overall calorimeter shape and dimensions.

II. Number of insulating wall layers inside the calorimeter to both allow for coolant passage (in order to cool the device), as well as to more efficiently insulate the water inside the tank from ambient thermal fluctuations. 
III. The materials to be used both for calorimeter tank construction, as well as the insulating material in between the layers to more effectively attain a quasi-adiabatic system.

IV. Sensitivity to coolant or ambient thermal variations.

As discussed previously, the inner MRI-linac and Gamma Knife unit bore and collimator ring (helmet) dimensions imposed a shape and maximum size restrictions on the calorimeter tank design. Once the shape and outer maximum dimensions of the calorimeter tank were decided (to accommodate both MRI-linac and Gamma Knife ${ }^{\circledR}$ ), a comprehensive set of simulations were undertaken to determine any advantages between our various considered designs addressing the second and third points above. We used Comsol Multiphysics ${ }^{\mathrm{TM}}$ software in order to solve the heat transport equation numerically inside a 2D axially symmetric geometrical model of our proposed experimental designs. Exploiting the symmetry definition (mirror application) can lead to less intensive computational simulations, since the number of degrees of freedom of the model directly affects the solution time. In our work, the general shape of the water calorimeter leads itself naturally to a 2D axially symmetric model, which helped in reducing the number of mesh elements required to describe the geometry.

In this work, heat transport due only to conduction was considered. Based on previous simulations and validations done by Sarfehnia et al [19], convective flow only becomes critical for calorimeter operations at room temperature or in presence of large temperature gradients often formed due to brachytherapy sources. It has been shown that at $4{ }^{\circ} \mathrm{C}$ operation temperature, convection can be ignored virtually in all external radiation beams [13]. This is both due to properties of water at this temperature as well as the extremely small radiation-induced temperature gradients that are formed as a result of high energy external photon therapy beams.

The calorimeter was designed to provide best thermal stability and reduce the effects of outside thermal variations on the water temperature of the inside the calorimeter. Through finite element modelling (FEM) of heat transport, the effects of several different 
parameters on thermal stability were studied. Several designs with different number of layers and insulator materials of varying thicknesses were investigated and optimized. Using the same modeling approach, the effectiveness of several insulating materials was studied to understand the best insulating medium for our application.

During the optimizations, we considered long term behavior of the system starting from a set of initial conditions (full equilibrium) and its behavior under realistic ambient temperatures, trying to quantify how close to an isolated adiabatic system we can get. Furthermore, we also studied the effects of various parameters on the behavior of the heat transfer correction factor. A large $k_{\mathrm{ht}}$ indicates significant deviations from ideal conditions, which in turn may suggest a larger dependency of the final dose uncertainty on accurate modeling of the setup conditions. Indeed, it is important to ensure robustness of the solution and ensure that the results are not affected significantly by minute changes in simulation details/initial conditions. A large variation in $k_{\mathrm{ht}}$ between subsequent simulated caloric runs in turn indicates a definite lack of robustness of the calorimeter results.

\subsubsection{Study One: Calorimeter Design Optimization}

To better illustrate the optimization process, four of the designs considered are noted in figures $4-4 a-d$. Figures $4-4 a, b, c$ show the cross-sections (design is axially symmetric around midline z-axis) of three designs that used two Lucite layers separated by an insulation layer (indicated in white), with coolants located in the first layer (actual tank wall). Figures 4-4b, c introduce an additional layer of air gap insulation with different widths, $3[\mathrm{~mm}]$ and $9[\mathrm{~mm}]$ respectively (indicated in grey) inside the calorimeter tank wall. In these designs that were intended to be rapid prototyped, the air gaps act as further thermal insulation. In these designs, the air gaps were not modeled/ included in the hemispherical portion of the tank as the Gamma Knife ${ }^{\circledR}$ beam will enter through the actual calorimeter wall from various directions, and we did not want to introduce further uncertainty in depth travelled by the beams in our design.

In figure 4-4d, an additional layer was modeled with coolant passing inside the second layer. This approach further insulates the water inside the tank not only from ambient 
conditions, but also from possible thermal variations of the coolant fluid itself. The diameter of the coolant passageway was taken to be $6[\mathrm{~mm}]$ in all cases.

(a)

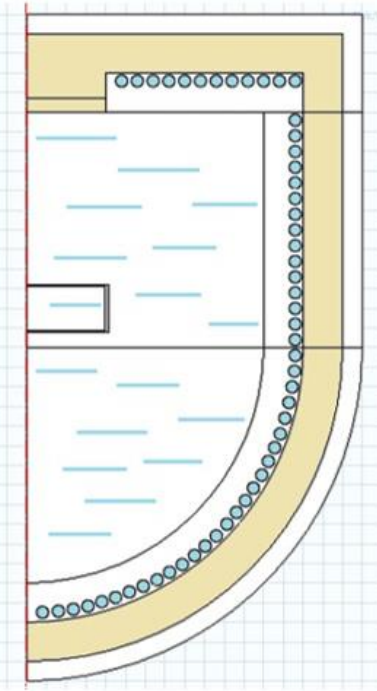

(b)

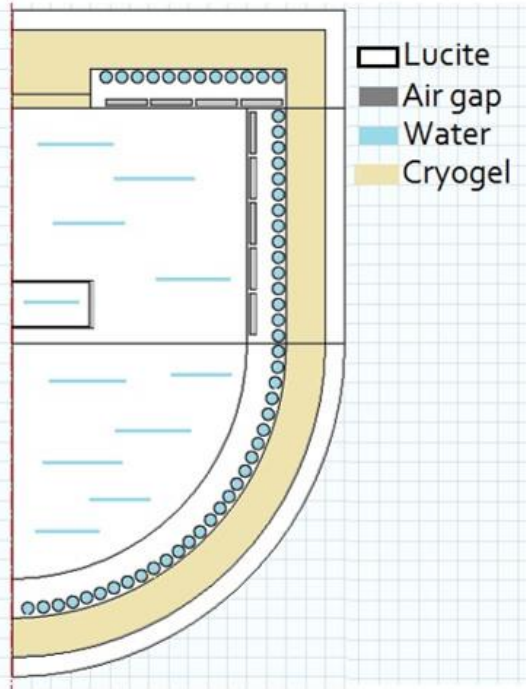

(d)

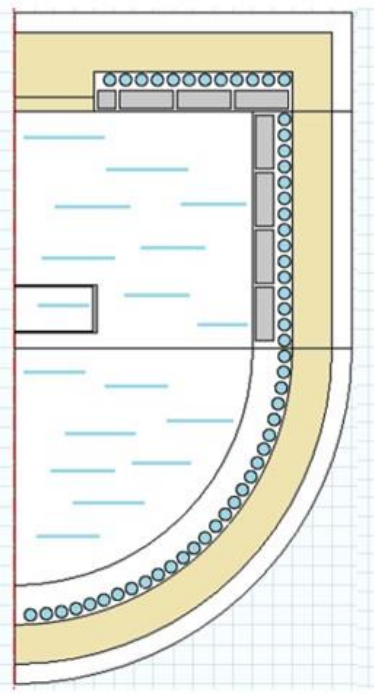

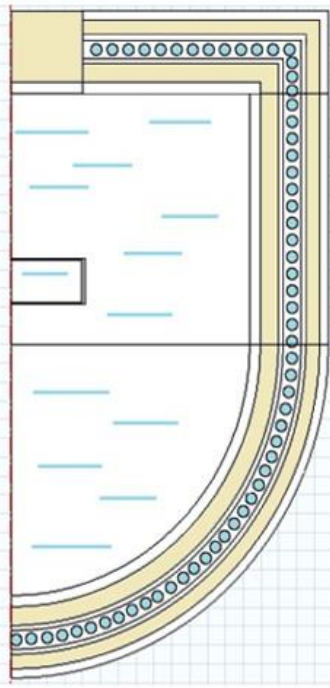

FIGURE 4-4 SEVERAL WATER CALORIMETER DESIGNS CONSIDERED IN THIS WORK. FROM LEFT TO RIGHT, THESE INCLUDE (A) A WC WITH COOLANT CHANNELS INSIDE THE CALORIMETER TANK WALL (B) SIMILAR TO (A) WITH 3MM THICK AIR GAP INSULATION (C) SIMILAR TO (A) WITH 9MM THICK AIR GAP INSULATION (D)THE LATTER WAS THE ONE SELECTED IN THIS WORK.

\subsubsection{Study Two: Insulation Material Optimization}

We also simulated and optimized three different types of insulation for the accepted design shown in figure 4-4d. Different insulations such as conventionally used Styrofoam®, air, and solid state thermal insulators such as Cryogel® were compared. Cryogel® is a carpet like, flexible aerogel with physical density of $160\left[\mathrm{Kg} / \mathrm{m}^{3}\right]$. It insulates the ambient temperature with thermal conductivity of $0.014[\mathrm{~W} / \mathrm{mK}]$. Cryogel ${ }^{\circledR}$ is only one of the many various products in the aerogel solid state insulator based family. Although used as thermal insulators in a wide range of applications from plumbing to NASA space suits, aerogel family of solid state insulators can be difficult to work with, and many versions are hygroscopic.

The material of choice for calorimeter wall simulation was Lucite®. Lucite is an MRI-safe plastic with physical density of $1180\left[\mathrm{Kg} / \mathrm{m}^{3}\right]$. 


\subsubsection{Study Three: Plane Parallel Vessel Optimization}

In this work, we optimized the dimensions of the plane parallel vessel. We changed the height, top and bottom glass window thicknesses, and radius of the glass vessel, to evaluate different designs based on similar criteria as before (including magnitude and variation in $\left.k_{\mathrm{ht}}\right)$.

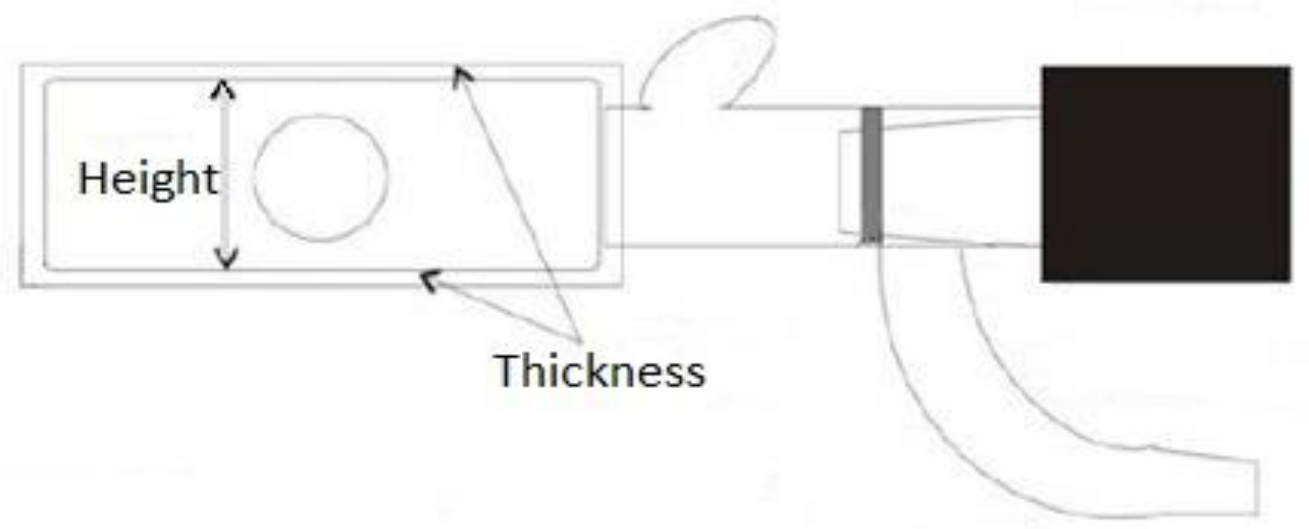

FIGURE 4-5 GLASS VESSEL. [13]. 


\section{CHAPTER 5 - RESULTS AND DISCUSSIONS}

\subsection{Concepts}

This chapter begins by describing various Comsol Multiphysics ${ }^{\mathrm{TM}}$ simulations and optimizations that are studied in this work, followed by the construction process of the inhouse built MRI-compatible water calorimeter. Finally, a set of preliminary measurements performed using the calorimeter under a high energy $6 \mathrm{MV}$ photon beam linear accelerator is presented.

\subsection{Study One: Calorimeter Design Optimization}

As described in Sections 4.1 and 4.3.1, following the decision to have the general shape of the water calorimeter to be cylindrical on the top with a hemispherical end on one side, several variations of the details within the design were considered. Figure 5-3a-d shows four such designs as described previously in section 4.3.1.1. In order to study the stability of the system, we assumed three cases: 1) Where the entire calorimeter is perfectly at thermal equilibrium at $4^{\circ} \mathrm{C}$, the coolant also passes through the calorimeter at a constant $4{ }^{\circ} \mathrm{C}$ without any temperature fluctuation, and the ambient temperature was kept at a constant $22^{\circ} \mathrm{C}$. In this way, we could observe which calorimeter best mimics an adiabatic system. 2) To further evaluate the system, we introduced a more realistic change in the above simulation where the coolant temperature increased as it travelled through the wall of the calorimeter. This was modelled through a relatively uniform rise in temperature of $0.02^{\circ} \mathrm{C}$ between the most extreme points of coolant. 3) Moreover, given the limitations of our fridge, in another set of simulations, we assumed that the coolant temperature is not maintained at a perfect $4^{\circ} \mathrm{C}$, rather we introduced a sinusoidal variation in the coolant temperature assuming worst-case expected variations in fridge performance. As such, a set of simulations were undertaken to evaluate the behavior of the calorimeter and the influence of coolant temperature variations on the caloric measurements, by simulating a cyclical (sinusoidal) coolant temperature variation with $1[\mathrm{~K}]$ amplitude and a period of $630[s]$. 
It should be noted, that although the starting conditions in these simulations are rather arbitrary, the point of the exercise is to evaluate the performance/behavior of the calorimeter tank under a few scenarios. By looking at the temperature drift curves, and their rate of change, one can evaluate how well the tank has been thermally insulated.

Figure 5-1 shows the simulation results for the temperature increase at the thermistor level (i.e. inside the vessel) for the first case described above. Looking at the slope of temperature drift curve, it is clear from the plot that the rate of heat drift for the three-layer system (5-3d) is the smallest. A small linear rate of change for the heat drift curve is always preferable as it can be more accurately accounted for through the extrapolation technique. It is noteworthy that our results also show that contrary to our hypothesis, the air gaps in the design do not help with the insulation and in fact allow for a greater conduction to take place.

Figure 5-2 shows the results for the second scenario discussed above. The results correspond to the heat drift at the point of measurement (i.e. where the thermistors would be) at the center of the vessel. Once again, as expected, the three-layer system performs dramatically better relative to the two layer insulations. Again, as mentioned previously, for the two layer systems, the air gaps inside the tank wall do not help with slowing down the conduction of heat from coolants to point of measurement. Indeed, the larger the width of the air gap, the greater the conduction of heat through.

Figure 5-3 shows the results based on the temperature fluctuations in water coolants assuming non-ideal fridge operation. As it can be seen in figure 5-3, the ambient temperature has the least effect at the point of measurement at the middle of the vessel on the 3-layer design (shown in black line). 


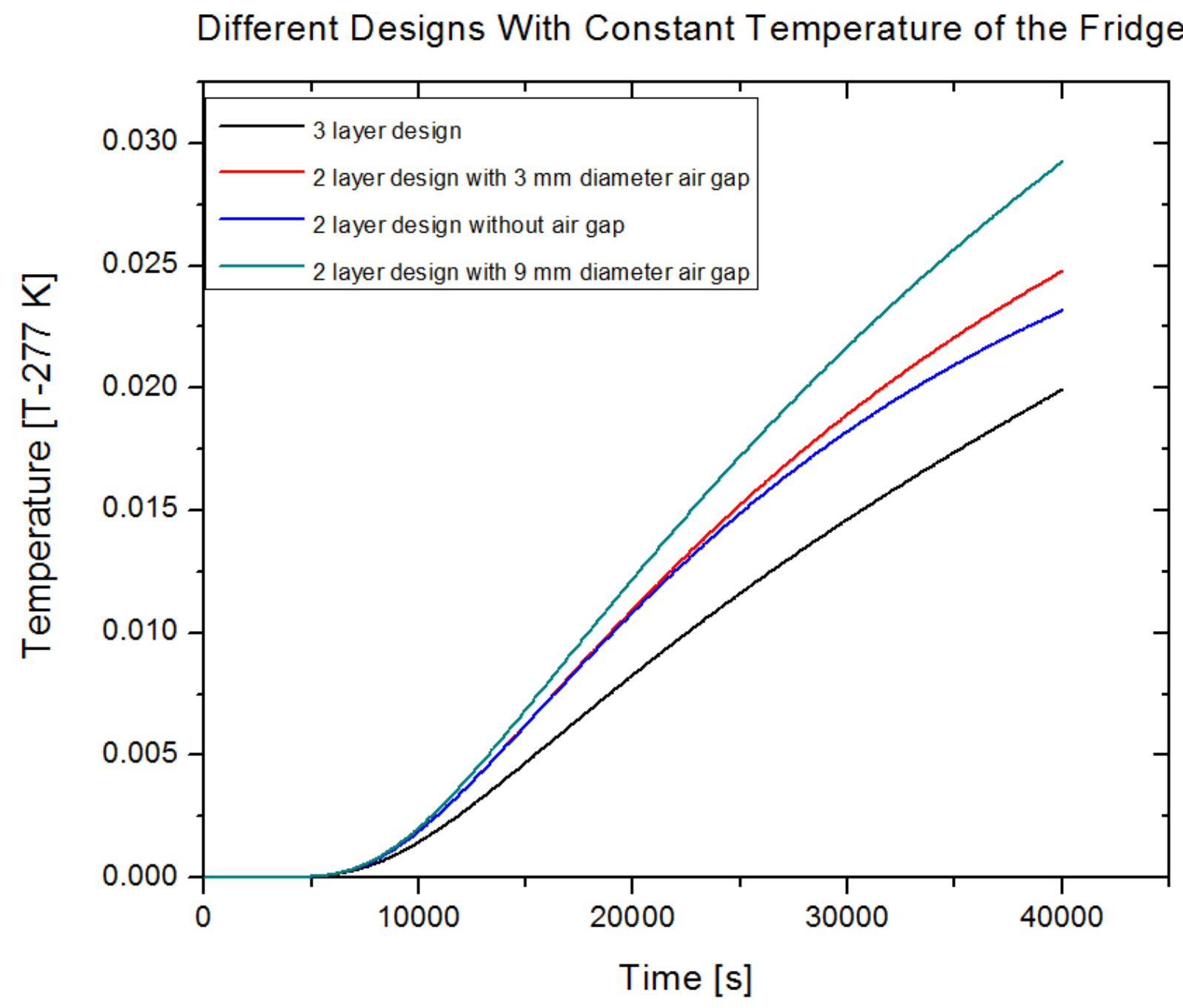

FIGURE 5-1RESULTS OF DIFFERENT DESIGNS WITH DIFFERENT LAYERS AND SIZE OF AIR GAPS FOR WATER CALORIMETER WITH CONSTANT TEMPERATURE AND WATER COOLANTS AT $4^{\circ} \mathrm{C}$ AND AMBIENT TEMPERATURE OF $22^{\circ} \mathrm{C}$. 


\section{Different Designs With Increasing Temperature of the Fridge}

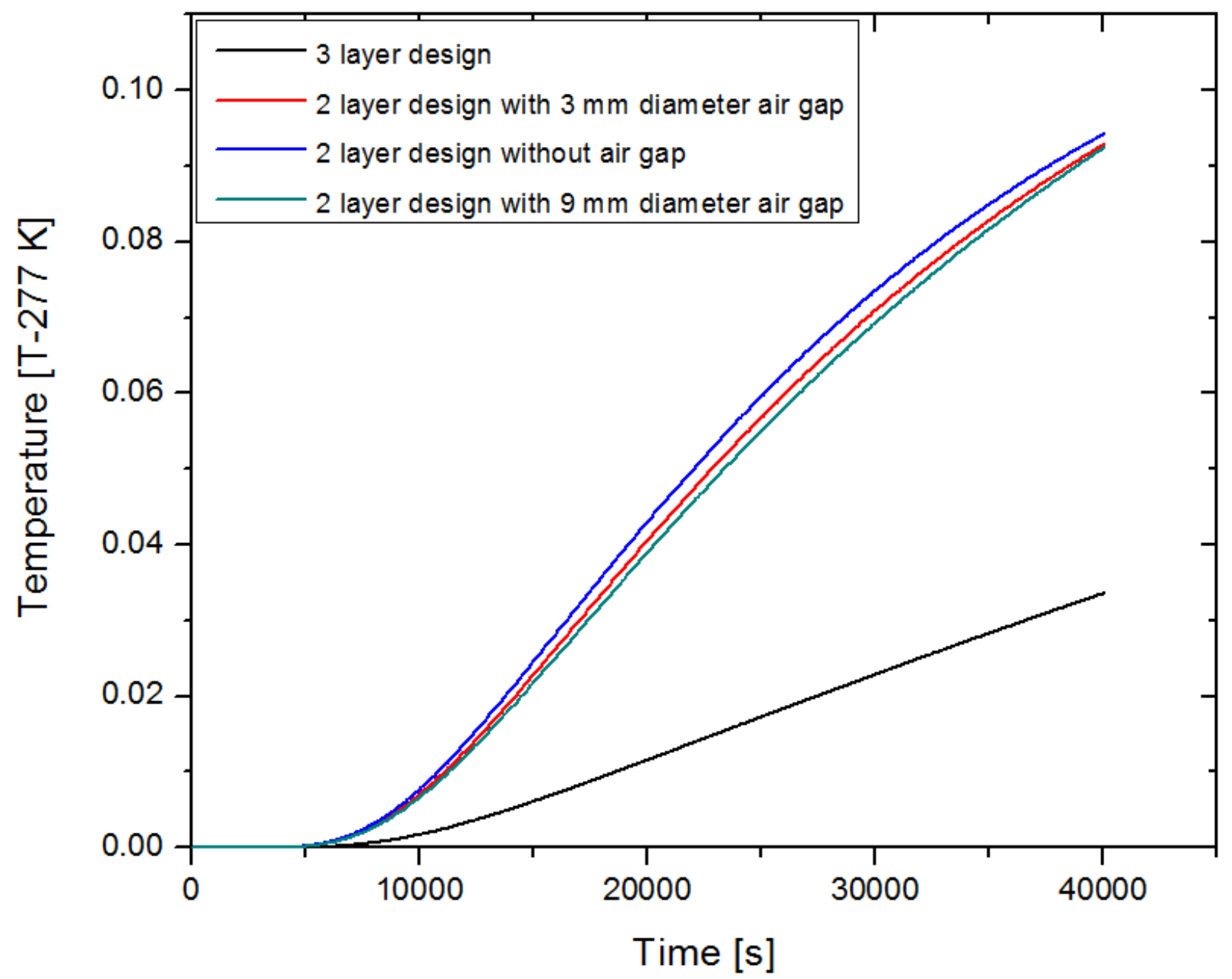

FIGURE 5-2 RESULTS OF DIFFERENT DESIGNS WITH DIFFERENT LAYERS AND SIZE OF AIR GAPS FOR WATER CALORIMETER WITH INCREASING TEMPERATURE AND WATER COOLANTS AT $4^{\circ} \mathrm{C}$ AND AMBIENT TEMPERATURE OF $22^{\circ} \mathrm{C}$. 


\section{Different Designs with Fluctuating Temperature of the Fridge}

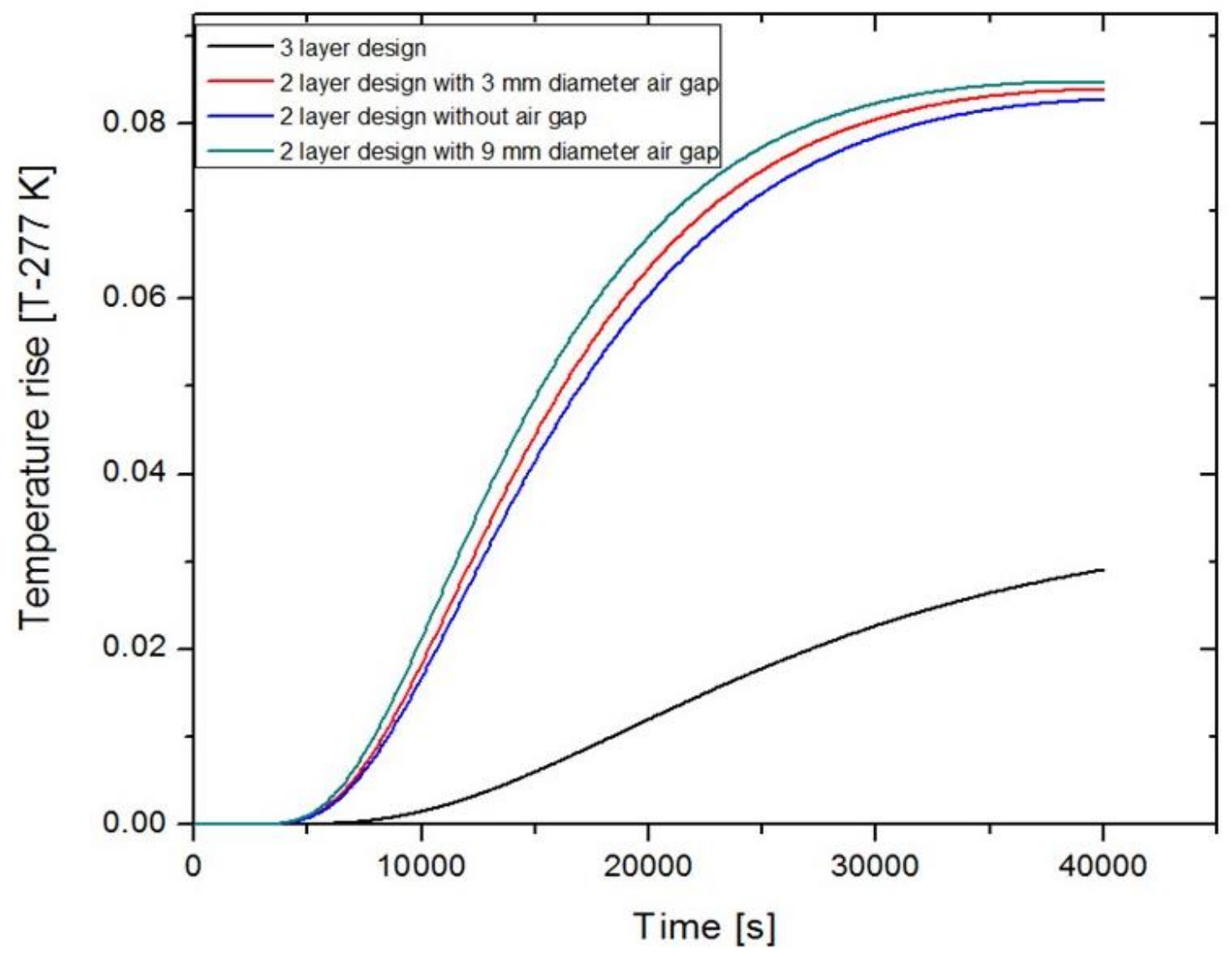

(a)

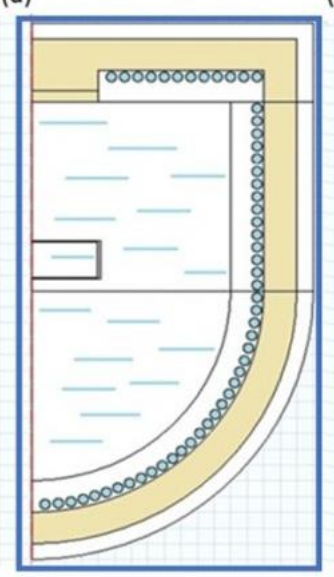

(b)

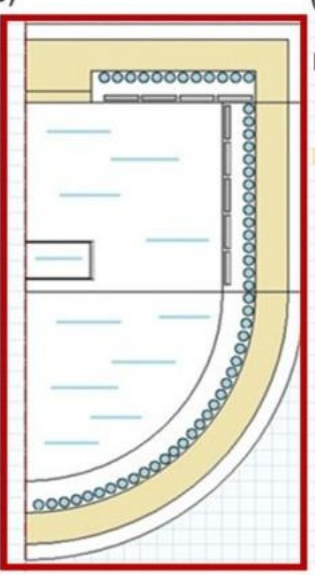

(c)

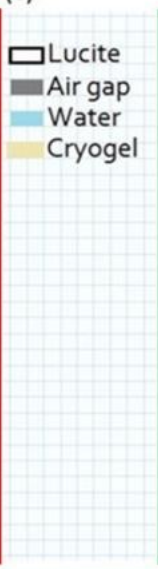

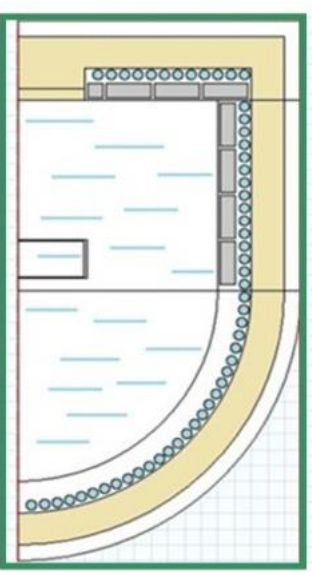

(d)

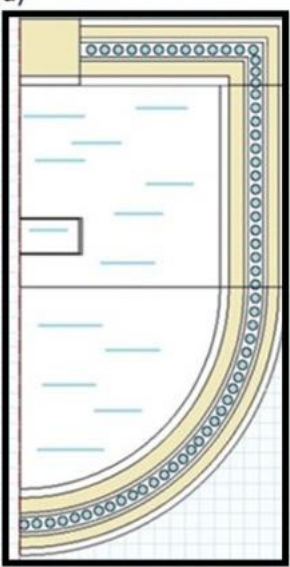

FIGURE 5-3 LONG TERM DRIFT CURVE SIMULATIONS RESULTS BASED ON THE TEMPERATURE FLUCTUATIONS IN WATER COOLANTS FOR SEVERAL WATER CALORIMETER DESIGNS (WATER COOLANTS AT $4^{\circ} \mathrm{C}$ AND AMBIENT TEMPERATURE OF $4^{\circ} \mathrm{C}$ ). FROM LEFT TO RIGHT, (A) COOLANT CHANNELS LOCATED IN THE WALL OF THE TANK WITHOUT AIR GAPS (B) COOLANT CHANNELS SEPARATED BY A 3MM THICK AIR GAP INSULATION FROM THE WATER TANK (C) COOLANT CHANNELS SEPARATED BY AN 9MM THICK AIR GAP INSULATION FROM THE WATER TANK (D) THE LATTER WAS THE ONE SELECTED IN THIS WORK WITH NO AIR GAPS AND WATER COOLANTS MOVED TO THE SECOND LAYER. 


\subsection{Study Two: Insulation Material Optimization}

We also simulated a long term (24 [hours]) drift curve for the selected 3-layer design shown in figure 5-3d, for three different insulation materials including Styrofoam ${ }^{\circledR}$ and air as well as Cryogel ${ }^{8}$ (shown in yellow in figure 5-3(d)) under two conditions: a) The entire calorimeter starting at $4^{\circ} \mathrm{C}$ initial condition, with the coolant and ambient temperature kept at a constant $4^{\circ} \mathrm{C}$ and $22^{\circ} \mathrm{C}$, respectively; b) The entire system starting at $4^{\circ} \mathrm{C}$, with the ambient temperature kept at a constant $22^{\circ} \mathrm{C}$, with the coolant warming from $4^{\circ} \mathrm{C}$ to $4.02^{\circ} \mathrm{C}$ as it travels down the calorimeter tank (figure 5-4a, b).

As it is shown in figure $5-4 a, b$, Cryogel ${ }^{\circledR}$ is significantly better thermal insulator relative to the other two commonly used thermal insulators. It can be seen clearly from the results, a system with Cryogel $\circledast$ insulation is much less prone to dramatic temperature variations at the measurement point. The Cryoge ${ }^{\circledR}$ drastically dampens the heat gain, resulting in much reduced rate of temperature change. In reality, given random environmental temperature fluctuations, a better insulator such as Cryogel@ would minimize the rate of heat drift at the point of measurement. The extrapolation results from the runs.

The smaller the rate of temperature change during a set of caloric measurement runs, the more robust the results of the measurements (i.e. the smaller the long-term drift temperature rise, the more robust the extrapolation of the pre-drift and post-drift to the mid-run would be since the results will be less prone to general longer-term drift). 
(a)

Different Insulations with Constant Temperature of Coolants

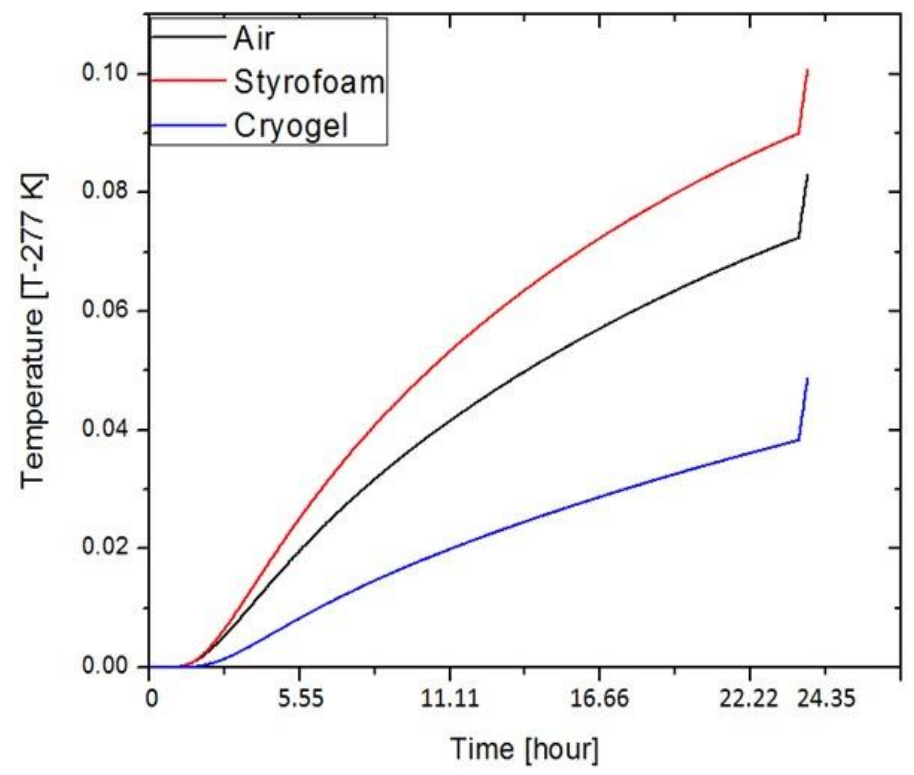

(b)

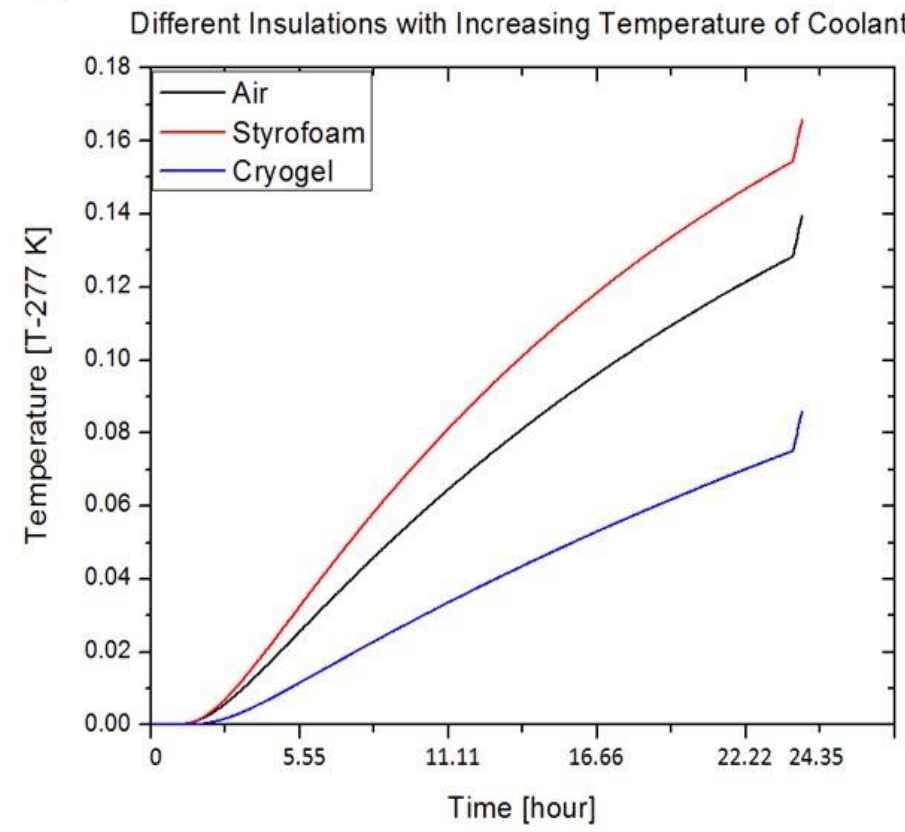

FIGURE 5-4 (A) DIFFERENT INSULATIONS ATTEMPTED AT THERMAL EQUILIBRIUM OF $4^{\circ} \mathrm{C}$ AND THE COOLANTS ALSO PASS THROUGH THE CALORIMETER AT A CONSTANT $4^{\circ} \mathrm{C}$ WITH THE AMBIENT TEMPERATURE OF $22^{\circ} \mathrm{C}(\mathrm{B})$

THE TEMPERATURE IN THE COOLANTS WARMS UP.

\subsection{Study Three: Plane Parallel Vessel Optimization}

\subsubsection{Thickness, Radius, Height Analysis}

In this work, we optimized the dimensions of the plane parallel vessel. We changed the height, top and bottom glass window thicknesses, and radius of the glass vessel, to find most optimal solutions (figure 5-5). Similar criteria with respect to analyzing both magnitude and variation in $k_{\mathrm{ht}}$ as described previously were employed in this section as well. 


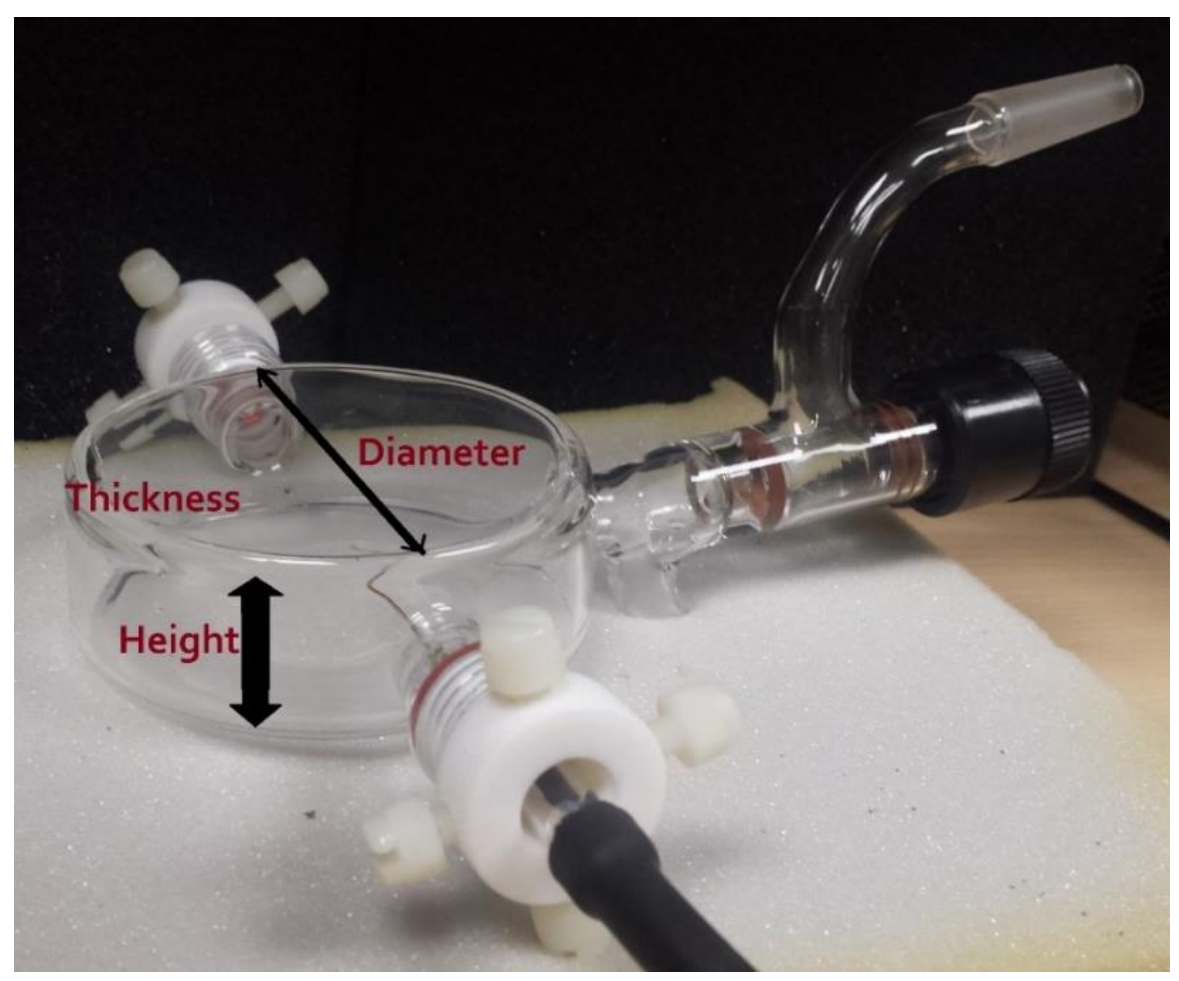

FIGURE 5-5 GLASS VESSEL, WITH ONE THERMISTOR DETECTOR POSITIONED INSIDE.

For any given simulation geometry (i.e. a given height, radius, and glass thickness), the very first simulation run shows the largest $k_{\mathrm{ht}}$ relative to subsequent radiation runs (for i.e. shown in figure 5-6). As part of our optimizations, we also evaluated the general standard deviation between ten simulated successive runs. For the MRL beam incident from top, glass thickness plays the largest effect on $k_{\mathrm{ht}}$ with variation of up to $0.6 \%$ in the $k_{\mathrm{ht}}$ of the first run for thicknesses ranging between 0.5-2.3 [mm]. Following the first (simulated) run, all subsequent runs in the series show a $k_{\mathrm{ht}}$ variation of only $0.1 \%$ with glass thickness (i.e. shown in figure 5-6). Other factors such as vessel radius and top/bottom glass separation have smaller effects on $k_{\mathrm{ht}}$. 


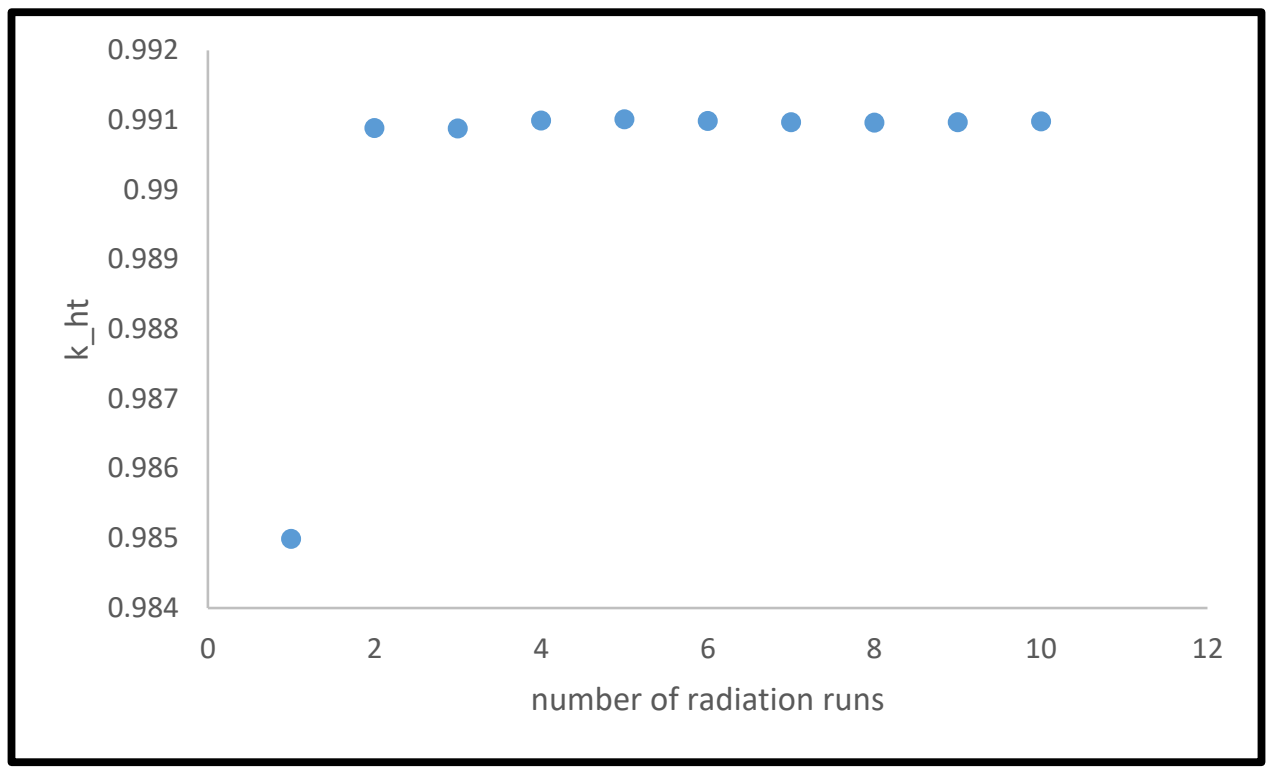

FIGURE 5-6 INDICATING THE FIRST AND SUBSEQUENT $k_{\mathrm{HT}}$ VERSUS RADIATION RUNS.

Figure 5-7 shows plots of heat transfer correction factor as a function of several parameters studied in this work. The simulations were performed for a $10 \times 10\left[\mathrm{~cm}^{2}\right], 6 \mathrm{MV}$ beam incident from the top on the vessel. Figure 5-7a shows average $k_{\mathrm{ht}}$ of 10 radiation runs for each glass thickness and height plane parallel vessel, while changing glass thickness in a range between 0.5-2.3 [mm], and height in a range between 15 to $35[\mathrm{~mm}]$ and keeping its radius fixed at $39.5[\mathrm{~mm}]$. As indicated in figure $5-7 \mathrm{a}$ the simulation with a radius of $39.5[\mathrm{~mm}]$, thickness of $0.5[\mathrm{~mm}]$, and height of $20[\mathrm{~mm}]$ has the closest heat transfer factor to unity. Based on our results, the optimal vessel thickness is $0.999^{*} 0.021$. As indicated in figure 5-7b, we kept the thickness fixed at 0.5 [mm] (the optimal glass thickness) and subsequently changed the range of height between 15-35 [mm], and radius $30-80[\mathrm{~mm}]$. Radius has the least effect on $k_{\mathrm{ht}}$, a maximum variation of $0.4 \%$ showed for consecutive runs for a given setup, as long as the radiation field size fully covers the entirety of the vessel (In this work, a $10 \times 10\left[\mathrm{~cm}^{2}\right]$ field size was simulated). As the result, based on our simulations, optimal vessel dimensions with $k_{\mathrm{ht}}$ closest to unity has a radius of $39.5[\mathrm{~mm}]$ and vessel height of $20[\mathrm{~mm}]$ for the glass thickness of 0.5 $[\mathrm{mm}]$. 
a)

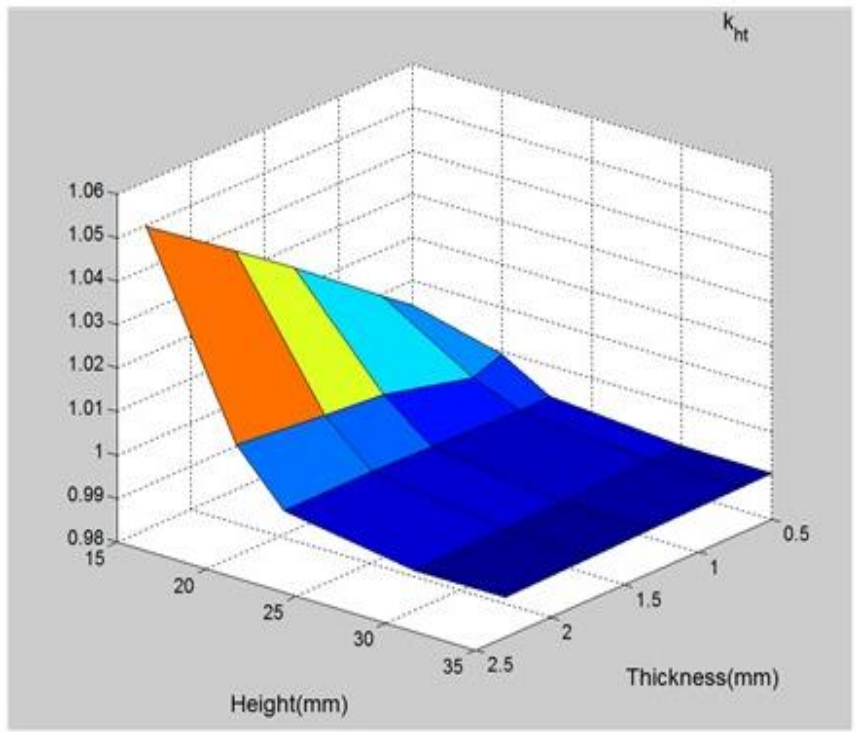

b)

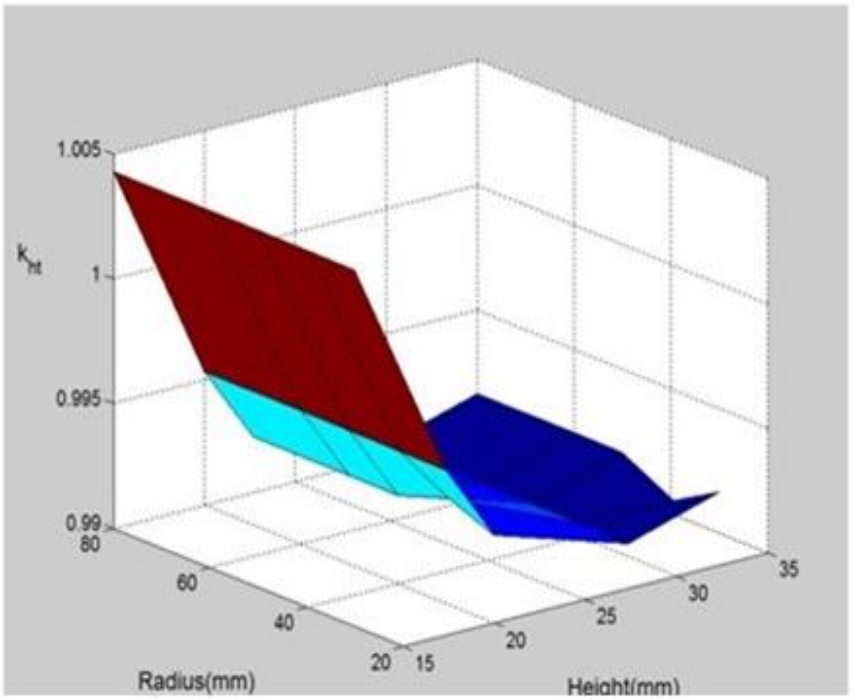

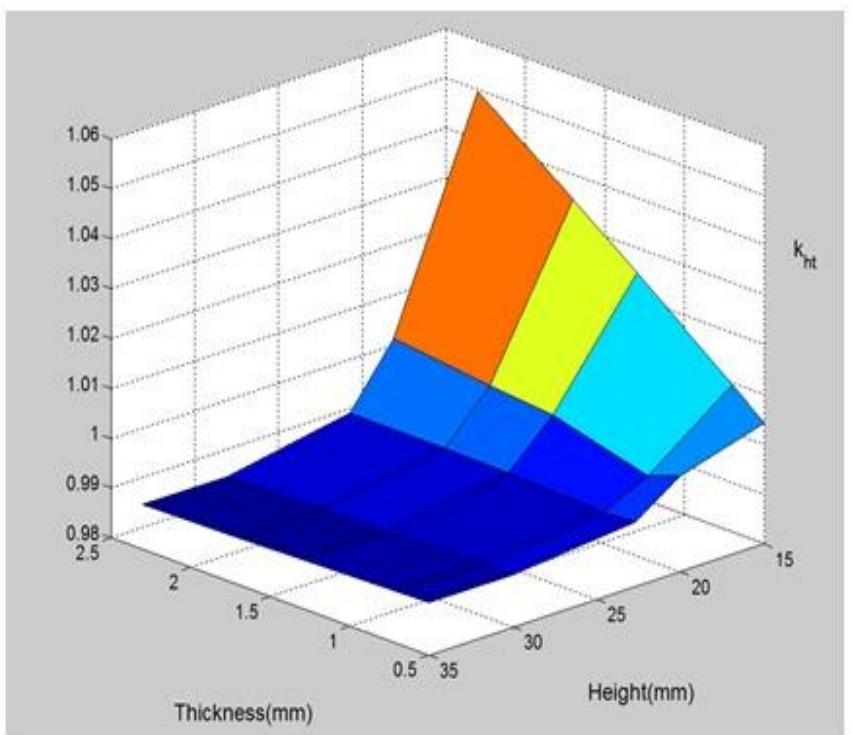

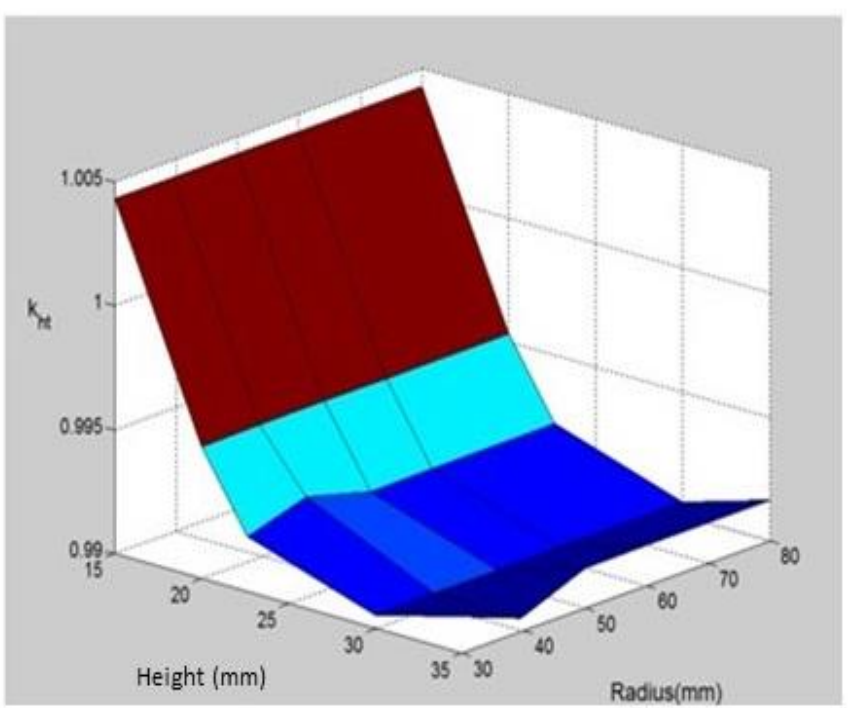

FIGURE 5-7 (A) HEAT TRANSFER CORRECTION FACTOR OF OPTIMIZATION OF THE HEIGHT AND THICKNESS OF THE PLANE PARALLEL VESSEL AT THE FIXED RADIUS OF 39.5 [MM] (B) OPTIMIZING THE HEIGHT AND RADIUS OF THE PLANE PARALLEL VESSEL AT THE FIXED THICKNESS OF 0.5 [MM].

Tables 5-1 and 5-2 indicate numerical results of the average $k_{\mathrm{ht}}$ of the optimization of plane parallel glass vessel with different heights, thicknesses, and radii. 
TABLE 5-1 AVERAGE $k_{\text {HT }}$ OF DIFFERENT THICKNESS AND DIFFERENT HIGHT FOR THE RADIUS OF 39.5 [MM] OF PLANE PARALLEL GLASS VESSEL.

\begin{tabular}{|c|c|c|c|c|c|}
\hline \multirow{2}{*}{ Height } & $\mathbf{1 5}[\mathrm{mm}]$ & $\mathbf{2 0}[\mathrm{mm}]$ & $\mathbf{2 2 . 6 6}[\mathrm{mm}]$ & $\mathbf{3 0}[\mathbf{m m}]$ & $\mathbf{3 5}[\mathrm{mm}]$ \\
\hline $0.5[\mathrm{~mm}]$ & 1.004 & 0.999 & 0.992 & 0.990 & 0.990 \\
\hline $0.7[\mathrm{~mm}]$ & 1.009 & 0.996 & 0.992 & 0.990 & 0.990 \\
\hline $1.3[\mathrm{~mm}]$ & 1.025 & 1.002 & 0.993 & 0.989 & 0.989 \\
\hline $1.7[\mathrm{~mm}]$ & 1.034 & 1.003 & 0.993 & 0.989 & 0.989 \\
\hline $2.3[\mathrm{~mm}]$ & 1.049 & 1.005 & 0.993 & 0.989 & 0.989 \\
\hline
\end{tabular}

TABLE 5-2 AVERAGE $k_{\text {HT }}$ OF DIFFERENT RADIUS AND DIFFERENT HIGHT FOR THE THICKNESS OF 0.5 [MM] OF PLANE PARALLEL GLASS VESSEL.

\begin{tabular}{|c|c|c|c|c|c|}
\hline Reight & $\mathbf{1 5}[\mathrm{mm}]$ & $\mathbf{2 0}[\mathrm{mm}]$ & $\mathbf{2 2 . 6 6}[\mathrm{mm}]$ & $\mathbf{3 0}[\mathrm{mm}]$ & $\mathbf{3 5}[\mathrm{mm}]$ \\
\hline $30[\mathrm{~mm}]$ & 1.004 & 0.995 & 0.992 & 0.990 & 0.991 \\
\hline $39.5[\mathrm{~mm}]$ & 1.004 & 0.999 & 0.992 & 0.990 & 0.990 \\
\hline $50[\mathrm{~mm}]$ & 1.004 & 0.995 & 0.992 & 0.990 & 0.991 \\
\hline $60[\mathrm{~mm}]$ & 1.004 & 0.995 & 0.992 & 0.991 & 0.991 \\
\hline $80[\mathrm{~mm}]$ & 1.004 & 0.995 & 0.992 & 0.990 & 0.991 \\
\hline
\end{tabular}

Standard deviation between runs also varied. Figure 5-8 shows plots of average standard deviation for 10 caloric runs as a function of several of parameters. Figure 5-8a shows average standard deviation for a plane parallel vessel by keeping its radius fixed at 39.5 $[\mathrm{mm}]$, while changing glass thickness in the same range between $0.5-2.3[\mathrm{~mm}]$, and height in a range between 15 to $35[\mathrm{~mm}]$. In figure $5-8 \mathrm{~b}$, the thickness is fixed at $0.5[\mathrm{~mm}]$ and subsequently changed the range of height between $15-35[\mathrm{~mm}]$, and radius $30-80$ 
[mm]. Finally, the vessel with radius of $39.5[\mathrm{~mm}]$ and vessel height of $22.66[\mathrm{~mm}]$ has the smallest standard deviation (by ignoring the height of $35[\mathrm{~mm}]$ ).

(a)
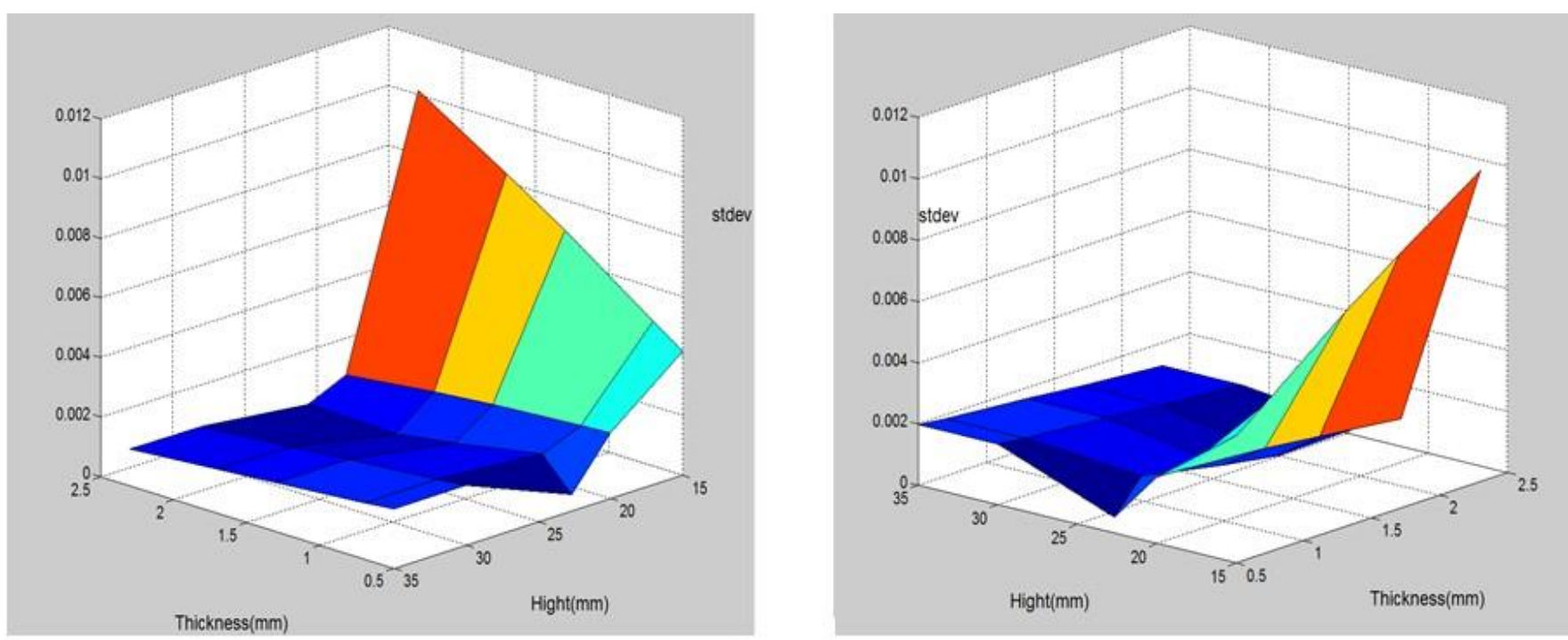

(b)
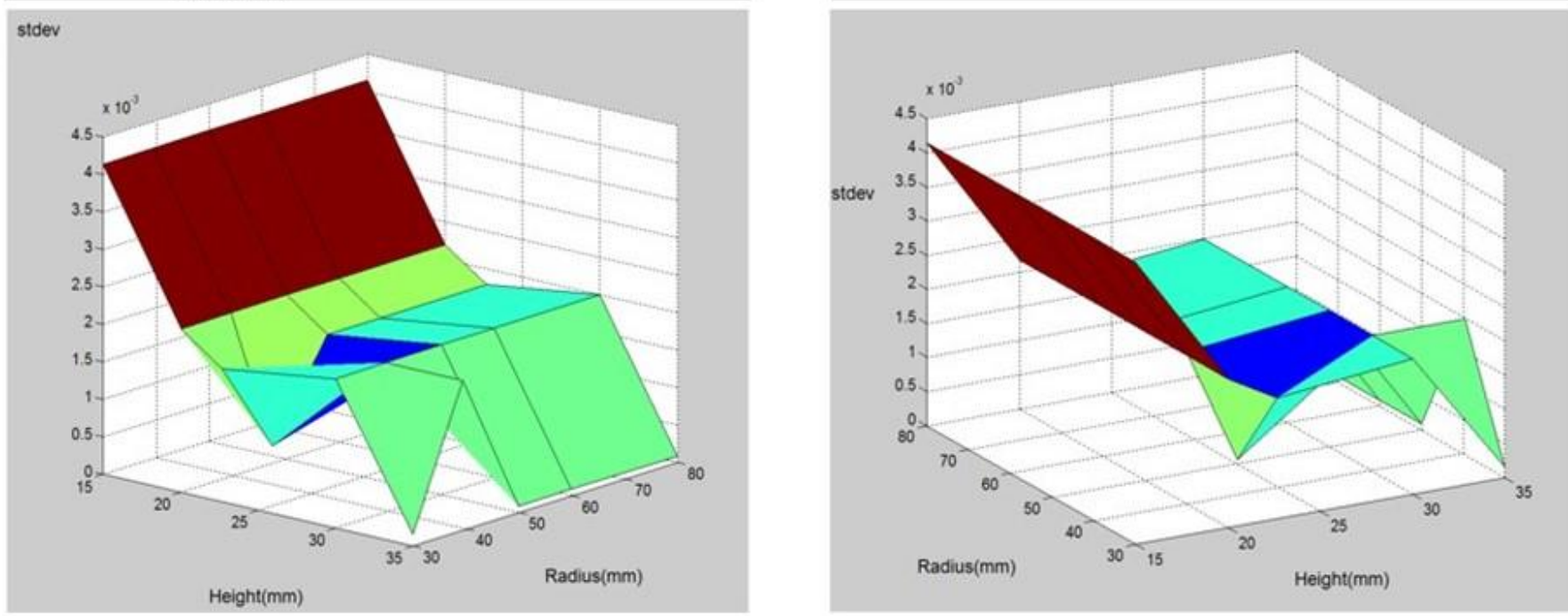

FIGURE 5-8 (A) STANDARD DEVIATION OF OPTIMIZATION OF THE HEIGHT AND THICKNESS OF THE PLANE PARALLEL VESSEL AT THE FIXED RADIUS OF 3.95 [CM] (B) OPTIMIZING THE HEIGHT AND RADIUS OF THE PLANE PARALLEL VESSEL AT THE FIXED THICKNESS OF 0.5 [MM].

Tables 5-3 and 5-4 indicates standard deviation of the $k_{\mathrm{ht}}$ between the 10 consecutive simulated runs for plane parallel vessel as a function of height, thickness, and radius. Standard deviations presented in these tables are normalized to average $k_{\mathrm{ht}}$ and expressed as a percentage. 
TABLE 5-3 AVERAGE PERCENTAGE STANDARD DEVIATION OF DIFFERENT THICKNESS AND DIFFERENT HEIGHT FOR THE RADIUS OF 39.5 [MM] OF PLANE PARALLEL GLASS VESSEL.

\begin{tabular}{|c|c|c|c|c|c|}
\hline $\begin{array}{c}\text { Height } \\
\text { Thickness }\end{array}$ & $\mathbf{1 5}[\mathrm{mm}]$ & $\mathbf{2 0}[\mathrm{mm}]$ & $\mathbf{2 2 . 6 6}[\mathrm{mm}]$ & $\mathbf{3 0}[\mathrm{mm}]$ & $\mathbf{3 5}[\mathrm{mm}]$ \\
\hline $0.5[\mathrm{~mm}]$ & 0.408 & 0.210 & 0.053 & 0.191 & 0.191 \\
\hline $0.7[\mathrm{~mm}]$ & 0.475 & 0.210 & 0.151 & 0.181 & 0.181 \\
\hline $1.3[\mathrm{~mm}]$ & 0.673 & 0.099 & 0.100 & 0.161 & 0.161 \\
\hline $1.7[\mathrm{~mm}]$ & 0.793 & 0.099 & 0.070 & 0.141 & 0.141 \\
\hline $2.3[\mathrm{~mm}]$ & 0.962 & 0.099 & 0.080 & 0.121 & 0.121 \\
\hline
\end{tabular}

TABLE5-4 AVERAGE PERCENTAGE STANDARD DEVIATION OF DIFFERENT RADIUS AND DIFFERENT HEIGHT FOR THE THICKNESS OF 0.5 [MM] OF PLANE PARALLEL GLASS VESSEL.

\begin{tabular}{|c|c|c|c|c|c|}
\hline $\begin{array}{c}\text { Height } \\
\text { Radius }\end{array}$ & $\mathbf{1 5}[\mathrm{mm}]$ & $\mathbf{2 0}[\mathrm{mm}]$ & $\mathbf{2 2 . 6 6}[\mathrm{mm}]$ & $\mathbf{3 0}[\mathrm{mm}]$ & $\mathbf{3 5}[\mathrm{mm}]$ \\
\hline $30[\mathrm{~mm}]$ & 0.412 & 0.218 & 0.178 & 0.201 & 0.0155 \\
\hline $39.5[\mathrm{~mm}]$ & 0.412 & 0.218 & 0.053 & 0.206 & $8.48 \mathrm{E}-05$ \\
\hline $50[\mathrm{~mm}]$ & 0.413 & 0.219 & 0.188 & 0.201 & $8.476 \mathrm{E}-05$ \\
\hline $60[\mathrm{~mm}]$ & 0.413 & 0.219 & 0.188 & 0.201 & $8.466 \mathrm{E}-05$ \\
\hline $80[\mathrm{~mm}]$ & 0.413 & 0.219 & 0.188 & 0.201 & $8.456 \mathrm{E}-05$ \\
\hline
\end{tabular}

\subsubsection{Thermistor Probes Position Sensitivity Analysis}

We also checked the variation of the heat transfer correction factor in different points of the plane parallel vessel with top and bottom window thickness of 0.7 [mm], side glass thickness of 1.96 [mm], radius of 39.5 [mm], and height of 22.66 [mm] (the glass vessel that we used in our measurements). Based on our results shown in figure 5-9 for different points from center to the right side of the plane parallel (from zero to $30[\mathrm{~mm}]$ ) vessel the 
variation of $k_{\mathrm{ht}}$ is unity. $k_{\mathrm{ht}}$ changes as the point gets closer to the side of the glass vessel. Figure 5-10 indicates the variation of $k_{\mathrm{ht}}$ at different points from bottom to the top of the glass vessel.

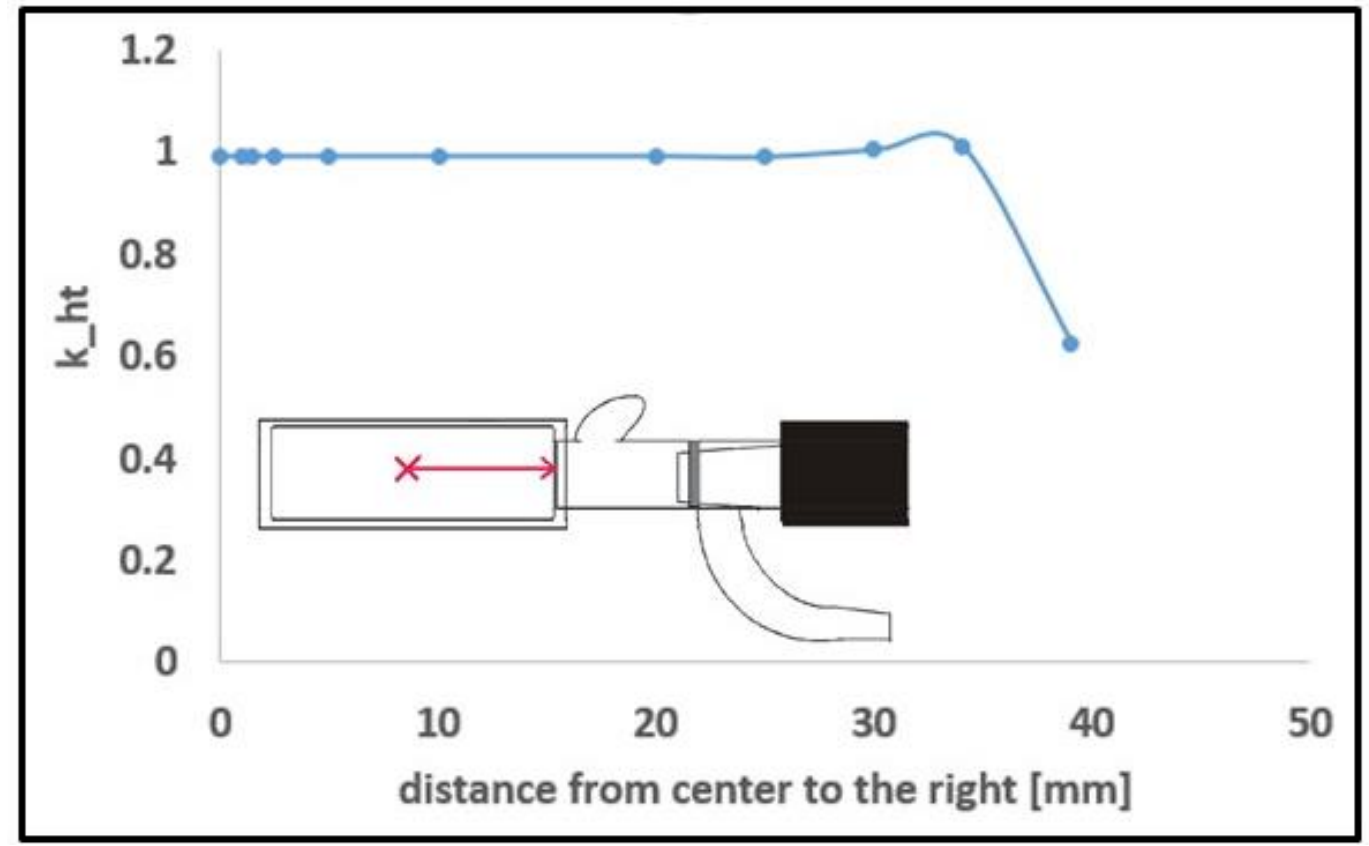

FIGURE 5-9 DIFFERENT POINTS FROM CENTER TO THE RIGHT SIDE OF THE GLASS VESSEL.

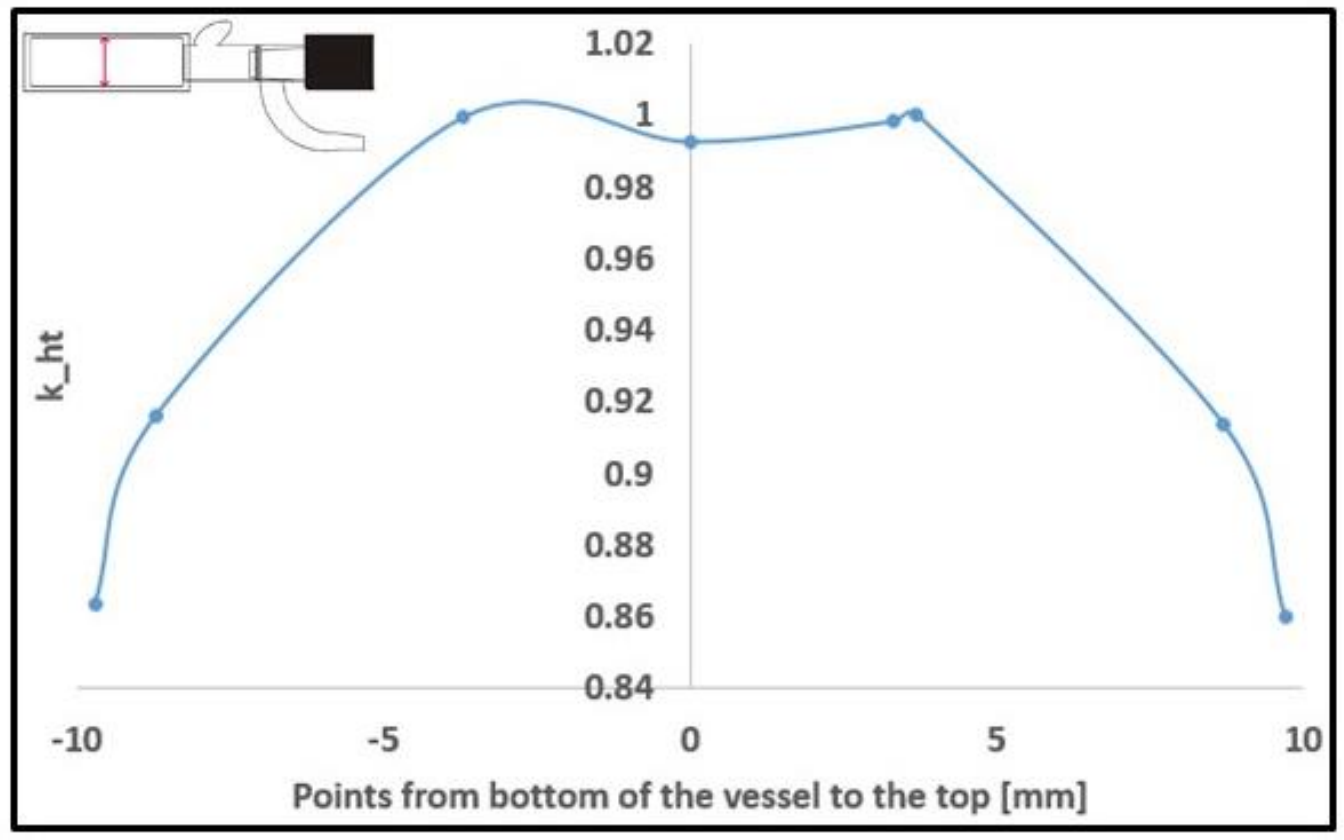

FIGURE 5-10 DIFFERENT POINTS FROM BOTTOM TO THE TOP OF THE GLASS VESSEL. 
TABLE 5-5 AVERAGE $k_{\text {HT }}$ AND AVERAGE \%STANDARD DEVIATION OF DIFFERENT POINTS IN PLANE PARALLEL GLASS VESSEL FROM RIGHT TO THE CENTER.

\begin{tabular}{|c|c|c|}
\hline $\begin{array}{c}\text { Distance } \\
\text { from right } \\
\text { to center }\end{array}$ & $\begin{array}{c}\text { Average } \boldsymbol{k}_{\text {ht }} \\
\text { at each } \\
\text { point }\end{array}$ & $\begin{array}{c}\text { Average } \\
\text { \%standard } \\
\text { deviation } \\
\text { at each } \\
\text { point }\end{array}$ \\
\hline $40.5[\mathrm{~mm}]$ & 0.598 & 0.294 \\
\hline $39[\mathrm{~mm}]$ & 0.624 & 0.230 \\
\hline $30[\mathrm{~mm}]$ & 1.004 & 0.214 \\
\hline $25[\mathrm{~mm}]$ & 0.991 & 0.096 \\
\hline $20[\mathrm{~mm}]$ & 0.992 & 0.158 \\
\hline $10[\mathrm{~mm}]$ & 0.992 & 0.159 \\
\hline $5[\mathrm{~mm}]$ & 0.992 & 0.159 \\
\hline $25[\mathrm{~mm}]$ & 0.992 & 0.159 \\
\hline $15[\mathrm{~mm}]$ & 0.992 & 0.159 \\
\hline $1[\mathrm{~mm}]$ & 0.992 & 0.159 \\
\hline $0[\mathrm{~mm}]$ & 0.992 & 0.159 \\
\hline
\end{tabular}

TABLE 5-6 AVERAGE $k_{\mathrm{HT}}$ AND AVERAGE \%STANDARD DEVIATION OF DIFFERENT POINTS IN PLANE PARALLEL GLASS VESSEL FROM BOTTOM TO THE TOP.

\begin{tabular}{|c|c|c|}
\hline $\begin{array}{c}\text { Distance from } \\
\text { bottom to top }\end{array}$ & $\begin{array}{c}\text { Average } \boldsymbol{k}_{\mathrm{ht}} \text { at } \\
\text { each point }\end{array}$ & $\begin{array}{c}\text { Average } \\
\text { \%Standard } \\
\text { deviation at } \\
\text { each point }\end{array}$ \\
\hline$-9.7[\mathrm{~mm}]$ & 0.863 & 0.054 \\
\hline$-8.7[\mathrm{~mm}]$ & 0.916 & 0.189 \\
\hline$-3.7[\mathrm{~mm}]$ & 0.999 & 0.317 \\
\hline $0[\mathrm{~mm}]$ & 0.992 & 0.159 \\
\hline
\end{tabular}




\begin{tabular}{|c|c|c|}
\hline $3.33[\mathrm{~mm}]$ & 0.998 & 0.301 \\
\hline $3.7[\mathrm{~mm}]$ & 1.000 & 0.327 \\
\hline $8.7[\mathrm{~mm}]$ & 0.913 & 0.192 \\
\hline $9.7[\mathrm{~mm}]$ & 0.860 & 0.054 \\
\hline
\end{tabular}

\subsection{Water Calorimeter Construction}

\subsubsection{Water Calorimeter Tank}

A detailed description of an in-house built water calorimeter with its materials is presented in this section. Figure 5-11 indicates a schematic diagram of the selected design of the new in-house built water calorimeter. As already described, the calorimeter unit is cylindrical with a hemispherical end, and is composed of three separate acrylic shells (see figure 5-3d for a cross section). The thicknesses of each cylindrical shell from the outermost to the innermost are: $6.17[\mathrm{~mm}], 3.11[\mathrm{~mm}]$, and $5[\mathrm{~mm}]$. The thickness of each shell in the hemispherical portion is 2.6 [mm]. The space between each subsequent shell is filled with a $10[\mathrm{~mm}]$ thick Cryogel ${ }^{\circledR}$ XT-E insulating material. The inner shell (radius $133.7[\mathrm{~mm}]$ and thickness of $5[\mathrm{~mm}]$ ) served as the actual water tank and housed the calorimeter's thermal detector. The middle shell was the cooling shell and consisted of one acrylic cylinder surrounded with superthane-ether tubing with 1.60 [mm] wall thickness encompassing the initial inner shell. The air gaps in between the cooling tubing were filled with a slow setting general purpose epoxy resin that was density matched to the acrylic cooling tubing. This was to create a smooth and uniform physical density equal to $1.3\left[\mathrm{~g} / \mathrm{cm}^{3}\right]$ for the beams incident from each direction in Gamma Knife ${ }^{\circledR}$. The cooling lid was attached to the entire tank using a series of plastic screws. 


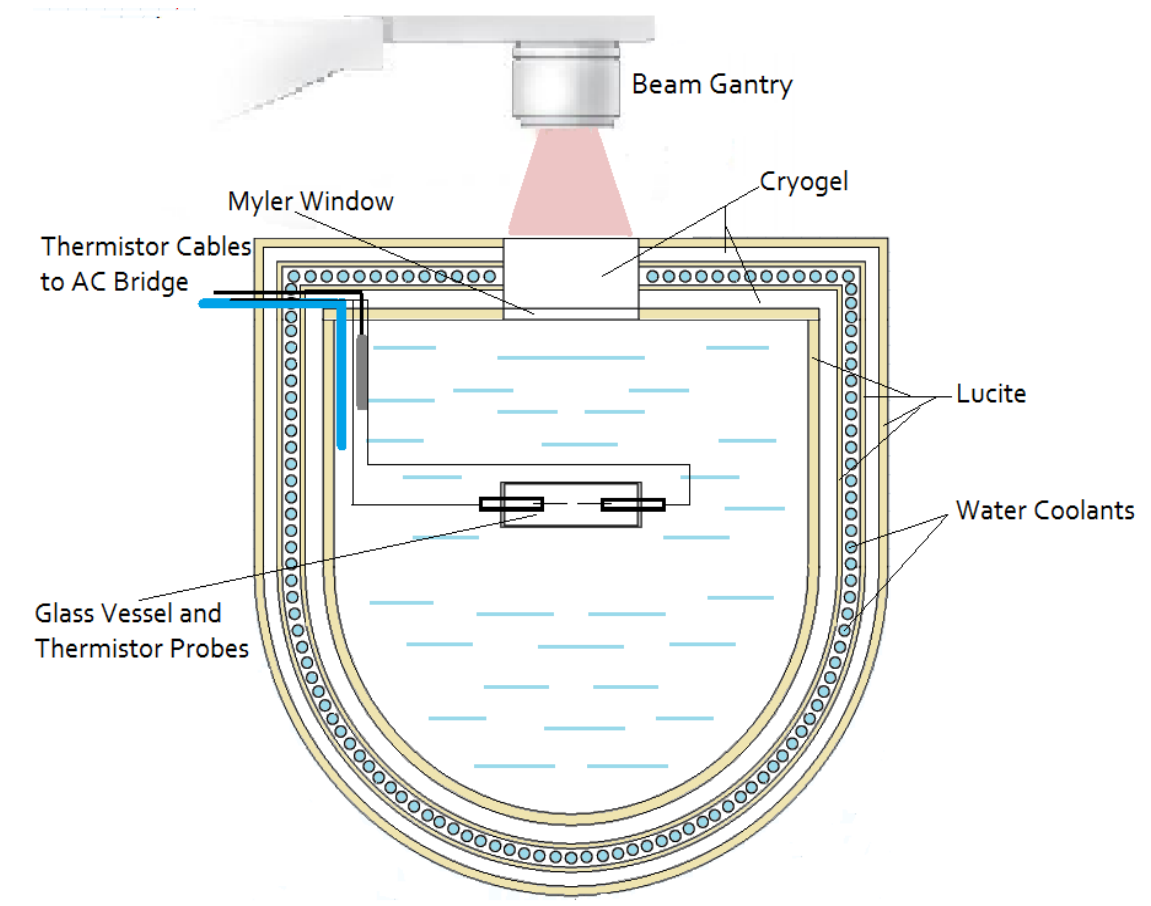

FIGURE 5-11 A schematic diagram of the water calorimeter positioned under a vertically incident radiation beam.

\subsubsection{Lid and Cooling System}

The cooling lid assembly (figure 5-13) consisted of two main components: 1) the lid itself, and 2) a cooling plate assembly. Two subcomponents of the lid assembly were the mylar window and its securing collar (which is where the radiation beam is directed) and a 10 $[\mathrm{mm}]$ thickness layer of Cryogel® insulation. The cooling plate was placed in the lid with the Cryoge $\AA^{\circledR}$ insulation layer separating the two. The lid also had two vertical up-stands that protruded through the cooling plate which enabled the filling and emptying of the water tank without any disassembly of the unit. The larger up-stand was for filling and emptying and the smaller one was an air vent. The cooling plate had two water inlets and two water outlets. One inlet-outlet pair was for allowing the flow of water through the lid assembly for cooling. The cooling water flows from the refrigeration unit to the lid's cooling plate inlet in series. After passing through the cooling plate outlet, the water then travelled via the Superthane ${ }^{\circledR}$ hose along the units cooling shell to cool the unit to $4^{\circ} \mathrm{C}$. It spiraled down the cylinder-hemisphere cooling shell to the center of the hemisphere where it loops and spirals back up the same shell exiting at the top to return to the refrigeration unit to 
be re-cooled before returning once again. The other inlet-outlet pair was used to drive two drive rotors to spin the stirring paddles; this was done in order to stir the water in the tank between calorimetric runs. A drive shaft was used to connect the drive rotor to the stir paddle. Although the rotors spin in the same direction, the stir paddles were mounted such that they were inverted (relative to each other), allowing rotational flow of water in the water tank for even stirring; this assists the water tank re-establish thermal equilibrium following irradiation. The mylar beam window was offset from the center of the lid by $49.90[\mathrm{~mm}]$ due to the size and orientation of the plane-parallel vessel used for temperature measurement. Measurement cables exited the phantom through an opening on the top corner of the lid.

The thermistor probes were encompassed by the plane-parallel glass vessel at the center of the water calorimeter. The glass vessel itself was secured in a detector holder, allowing a small degree of rotational adjustment around both the $\mathrm{x}$ and $\mathrm{y}$-axes using adjustment screws. In order to position the vessel accurately, the detector holder itself was secured to a detector attachment plate, which was fixed to the inner wall of the water tank (figure $5-12 b, c)$.

The entire calorimeter unit was comprised of non-metallic materials such as Acrylic, Delrin, Nylon, PEEK (Poly Ether Ether Ketone), polycarbonate, Starboard and ceramic in order to preserve MRI compatibility. All fasteners were either nylon or PEEK, and the total calorimeter tank unit dimensions were $375[\mathrm{~mm}]$ in diameter by $410.80[\mathrm{~mm}]$ in height (excluding the filler cap). 

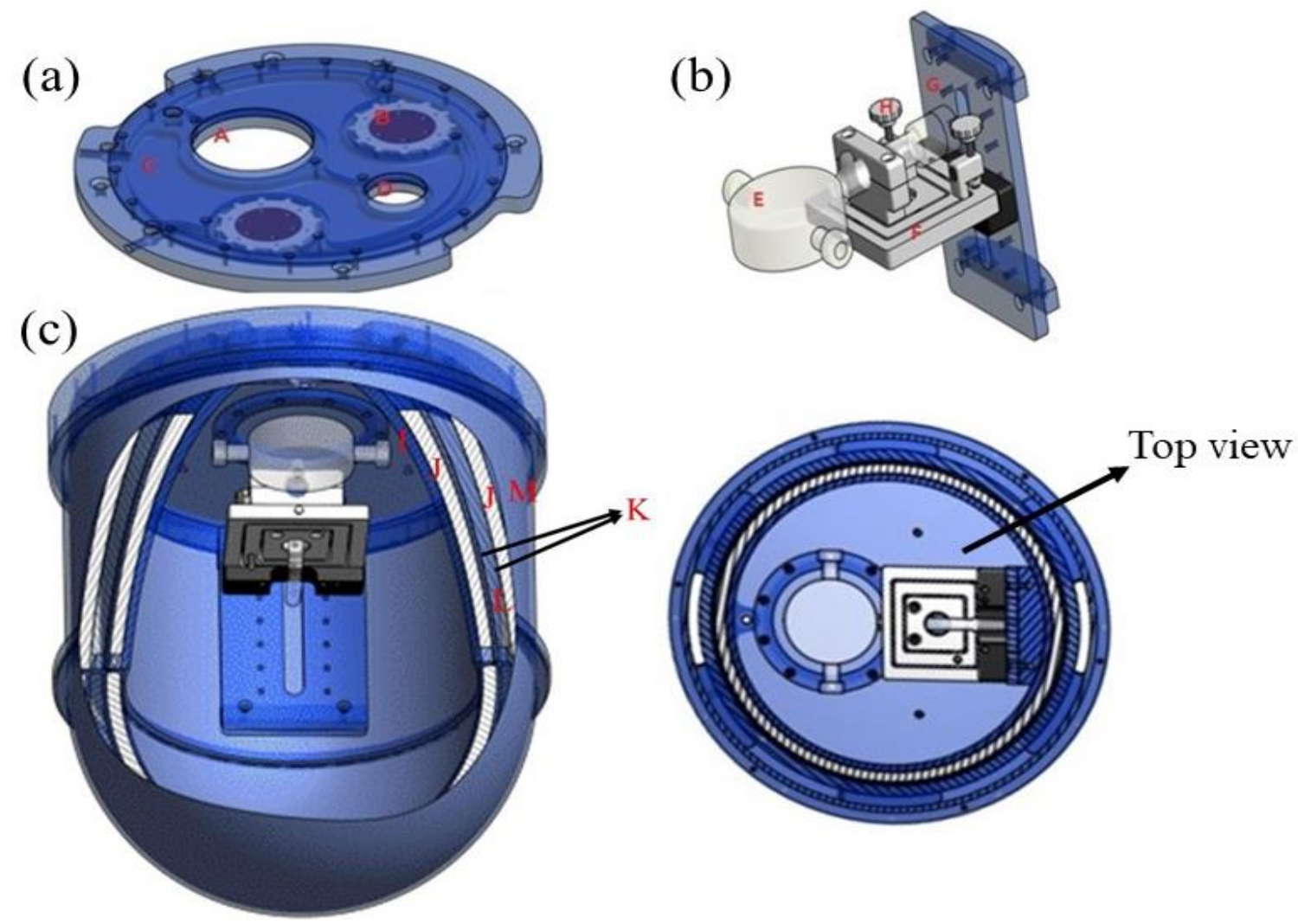

(a) Cooling lid

A. $50[\mathrm{~mm}]$ radius window for radiation to enter calorimeter (sealed by a 0.25 [mm] thin mylar sheet)

B. Drive rotors for stirring operated hydraulically

C. Lid coolant plate

D. Opening to allow for filling/emptying the water tank

(b) Holder
E. Glass vessel
F. Detector holder
G. Detector attachment plate
$\mathrm{H}$. Adjustment screws

(c) Water phantom

I. Primray $5[\mathrm{~mm}]$ Lucite wall

J. $10[\mathrm{~mm}]$ Cryogel $\circledast$ (indicated in white color)

K. $3.11[\mathrm{~mm}]$ Lucite layers housing water coolants

L. $10[\mathrm{~mm}]$ water coolants

M. $6.17[\mathrm{~mm}]$ housing Lucite

FIGURE 5-12 THIS FIGURE INDICATES A CAD DESIGN OF (A) THE COOLANT LID, (B) THE DETECTOR HOLDER AND (C) CROSS SECTION OF THE IN-HOUSE BUILT WATER CALORIMETER. 


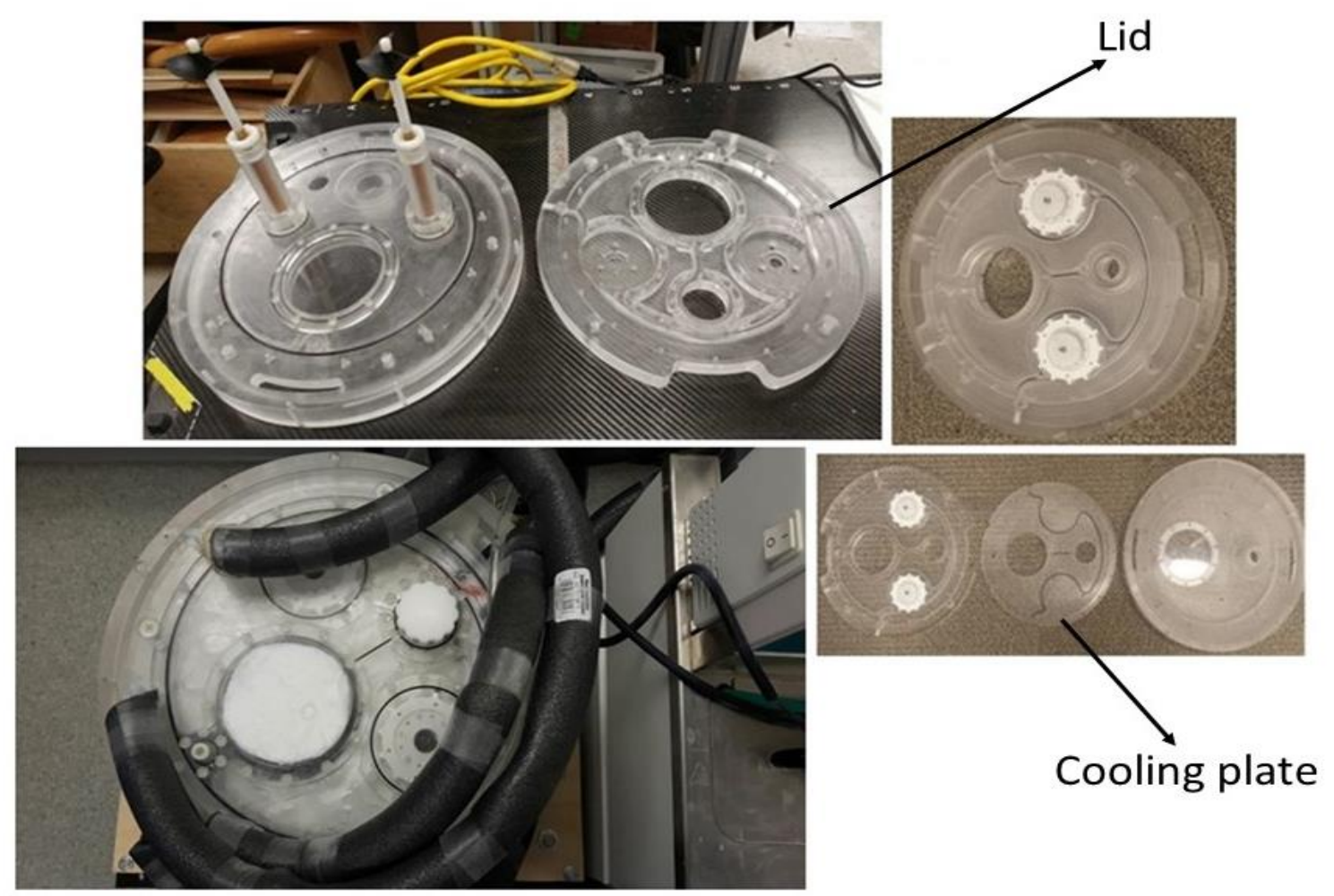

FIGURE 5-13 3-layer water calorimeter lid with stir puddles.

\subsection{Preparation for Measurements}

\subsubsection{Vessel}

To measure absolute dose by means of temperature sensitive thermistor detectors encompassed inside a glass vessel, we need to minimize radiation-induced chemical reactions that occur in presence of impurities in water (discussed in detail in Section 3.5) by using ultra-pure water.

The glass vessel pre-measurement preparation process is explained below: In the first step, we wash the vessel and thermistor probes thoroughly. We place and adjust the calibrated thermistors inside the clean vessel. The thermistor probes are inserted through the ports on each side of the vessel (5-14). These ports are sealed with CAPFE $\AA$ Teflon encapsulated O-rings to seal the high pure water and keep it inside the vessel. The two probes are positioned with $2-3[\mathrm{~mm}]$ separation between their tips and 
are positioned on the vessel's central axis. The height of the thermistor tips with respect to the top glass window of the vessel is measured with a traveling microscope.

The vessel is subsequently filled with ultra-pure water (organic content $<2 \mathrm{ppb}$ ) obtained from a Millipore water purification system. To remove any dissolved gases inside the high purity water, the water is subsequently bubbled with high purity hydrogen $(99.999 \%$ purity) for roughly three hours. After finishing the bubbling process a small gas bubble can be trapped inside the small glass bulb on the vessel arm.

This approach effectively saturates the pure water with hydrogen gas (replacing any dissolved gases) for which heat defect has been well studied, and understood to be negligible (section 3-5).

The bubbling process is performed by inserting a flexible acrylic tube down inside the vessel (Fig 5-15). Following the full saturation of the system with pure hydrogen gas, the vessel is fully sealed closing the glass stopcock (figure 5-14).

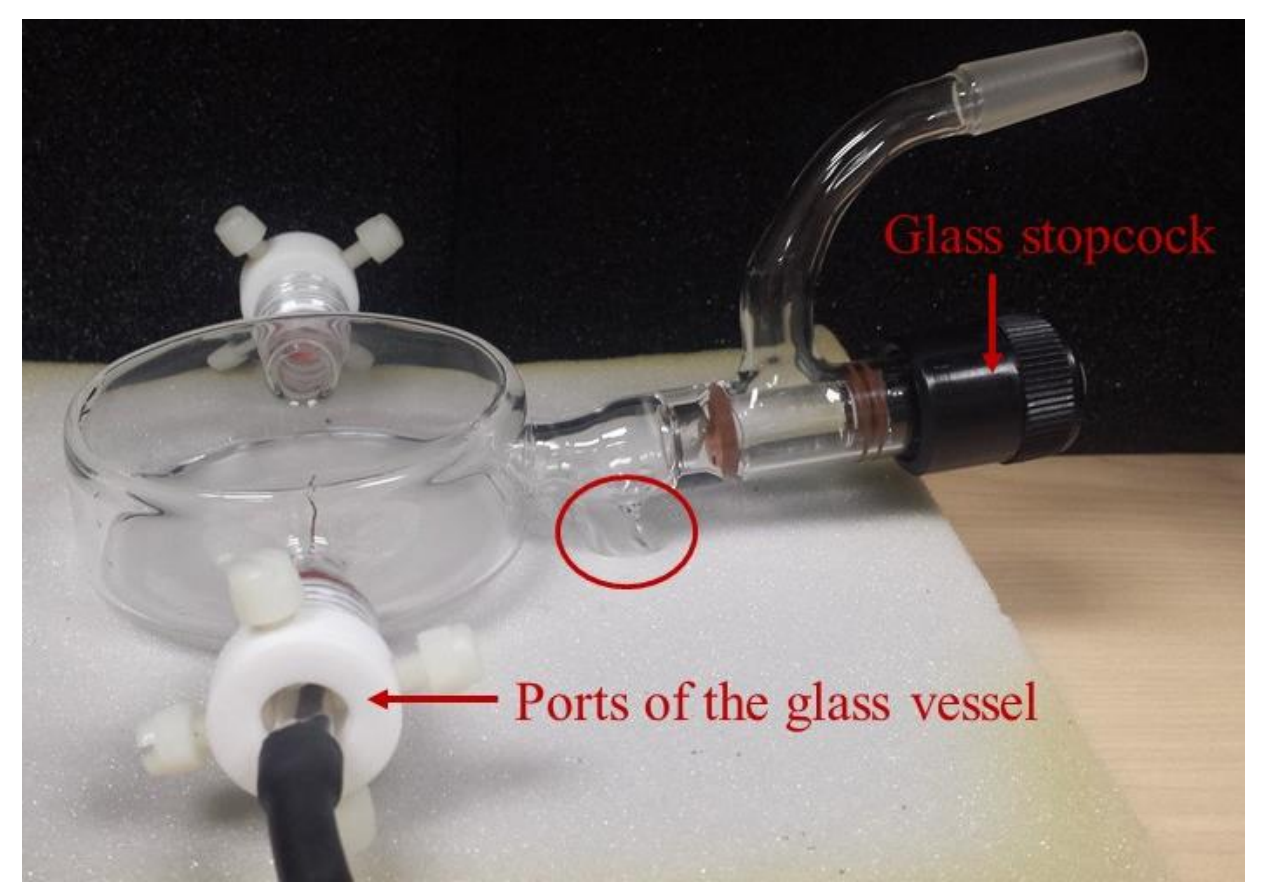

FIGURE 5-14 THERMISTOR DETECTORS ARE INSERTED THROUGH THE PORTS ON EACH SIDE OF THE VESSEL. THE VESSEL'S SMALL GLASS BULB IS INDICATED IN THE RED CIRCLE. 


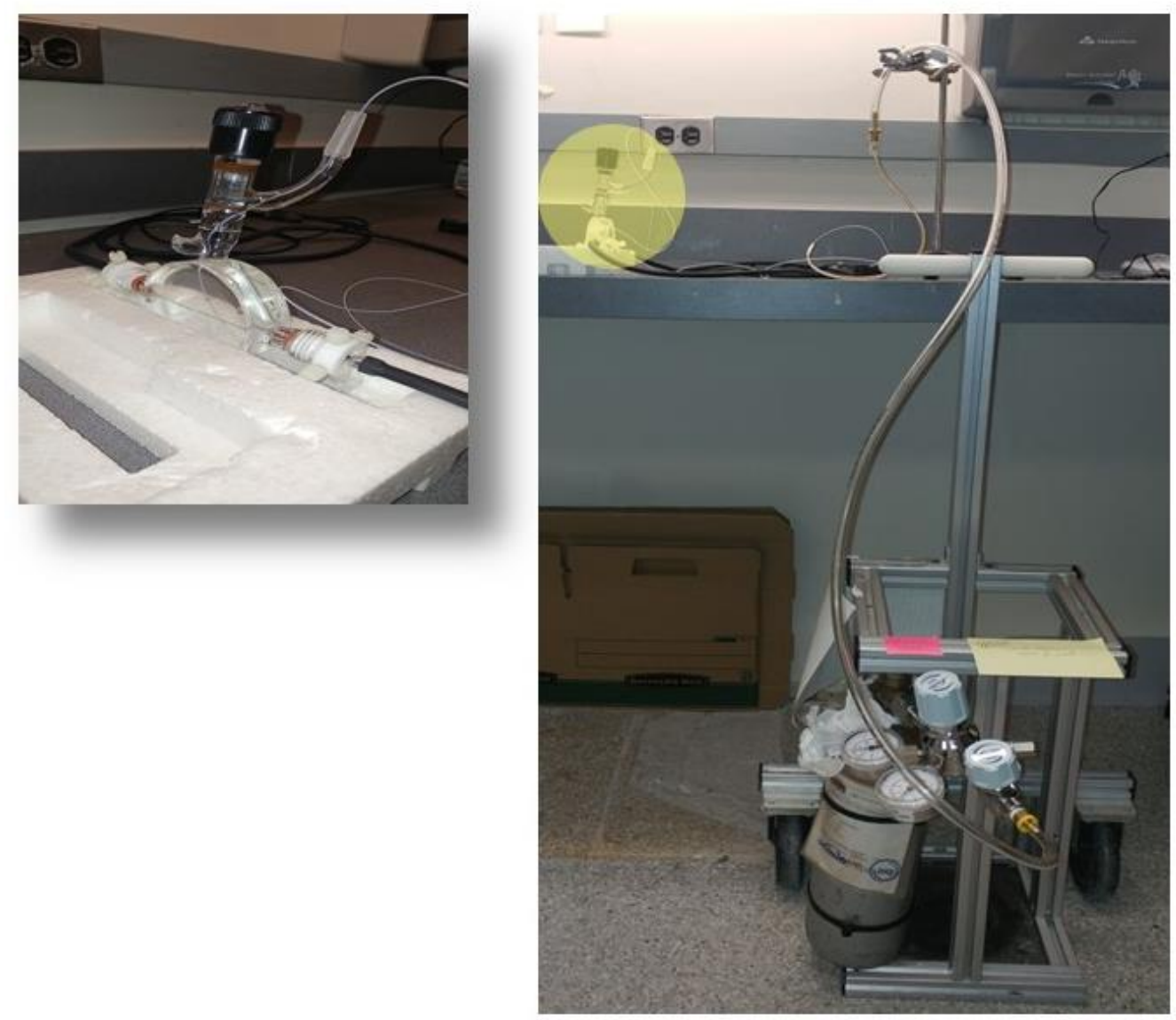

FIGURE 5-15 Vessel bubbling process with pure Hydrogen

This procedure avoids the onset of radiation-induced chemical reactions that occur in the presence of impurities in water (previously discussed in section 3.5).

\subsubsection{RTD and Thermistor Probe Calibration}

Both The PT-100 RTD temperature probes as well as the sensitive measuring thermistors were calibrated off-site for temperature changes from $-4^{\circ} \mathrm{C}$ to $+12^{\circ} \mathrm{C}$ according to the process described in section 3.3.2. From the analysis of this calibration, the resistance as a function of temperature was determined and a function was fit to the data [13].

\subsubsection{Ohm Calibration}

Ohm calibration runs were performed over the course of the measurements. They allow us to evaluate the relation of the generated voltage by a $1[\Omega]$ out of balance resistance (Note that $1[\Omega]$ typically corresponds with roughly a 1 [Gy] radiation dose). The Ohm 
calibrations are performed in between radiation runs as the results depend on calorimeter temperature. They are obtained by simply measuring the voltage change not as a result of radiation, but as a result of changing the balanced bridge by $1[\Omega]$ using the decade resistor box (burster) [13].

\subsection{Water Calorimeter Set up}

To prepare for the measurements, the glass vessel was secured using a detector holder to the inner wall of the calorimeter tank. The holder enables a small degree of rotational adjustment around both the $x$ and $y$-axes using adjustment screws.

For the glass vessel leveling a jig was used to ensure that the vessel is both level with respect to surface of the mylar entrance window and is centered directly underneath (figure 5-16c). Once leveled, the distance from the vessel's top surface to the Mylar

window is measured and recorded. The thermistor leads as well as the cables from the RTD probes placed inside the calorimeter are all then fed through the appropriate holes in the lid (figure 5-16d).

Once the lid is secure, the water is filled inside the tank, and the cooling process began (figure 5-17b).

The entire system was cooled with Neslab RTE-7 fridge for a minimum of 72 hours, with the water going through the water tank and the lid. The water inside the water tank was stirred with stir paddles during the cooling process and between time intervals to reestablish the thermal equilibrium in water. Figures 5-16 and 5-17 indicates the process of water calorimeter set up. 
(a)

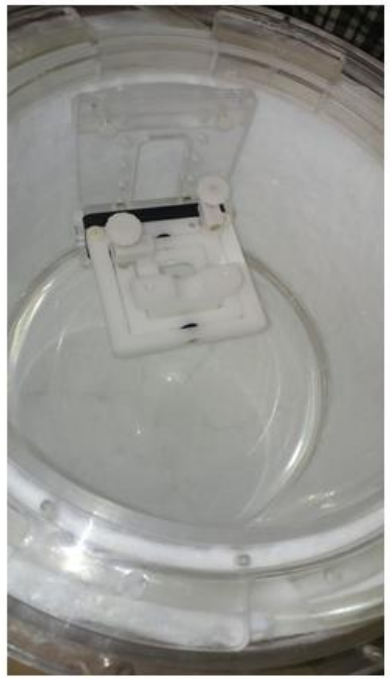

(b)

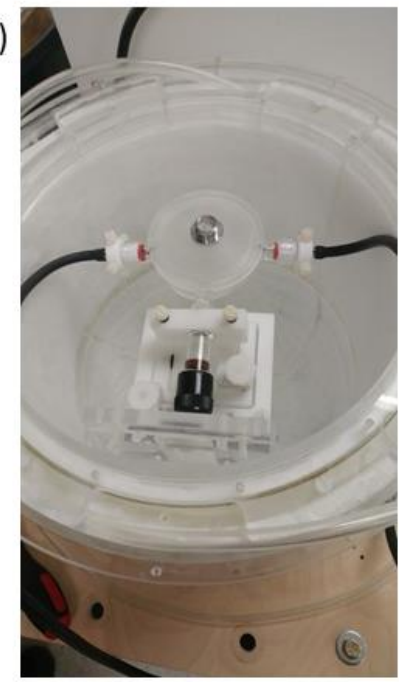

(c)

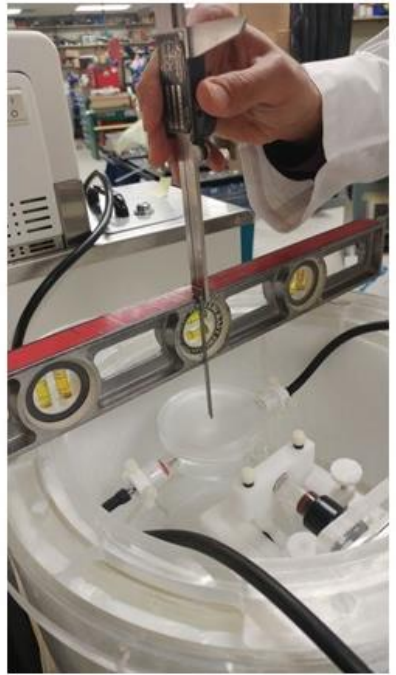

(d)

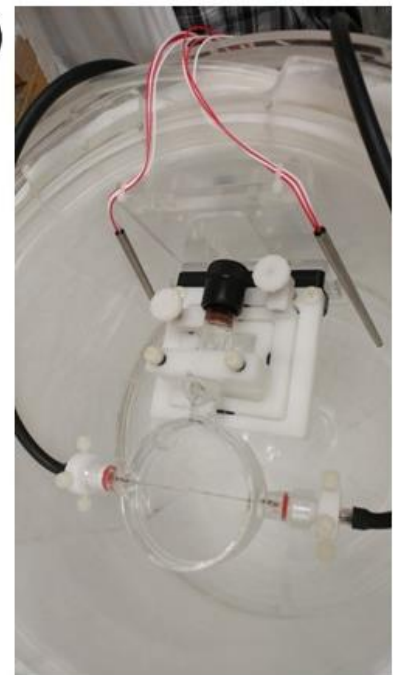

Figure 5-16 (A) Holder ATTACHEd ON THE SIDE OF THE TANK (B) LEVELING THE GLASS VESSEL (C) VESSEL AND TANK ALIGNMENT (D) RTD PROBES SECURED TO DETECTOR ATTACHMENT PLATE.

(a)

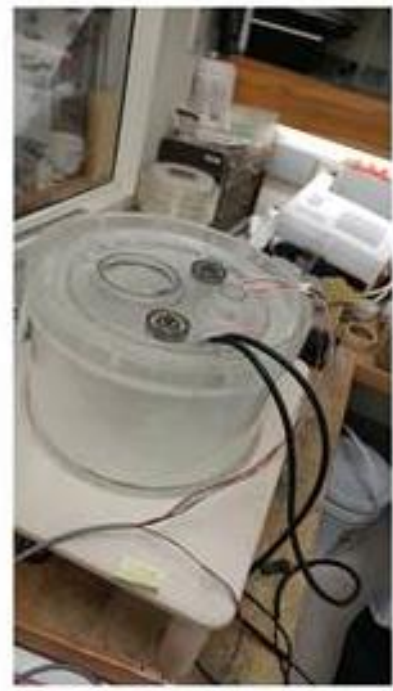

(b)

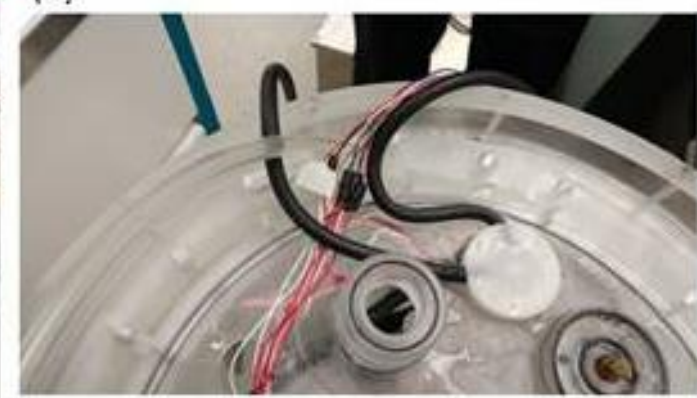

(c)

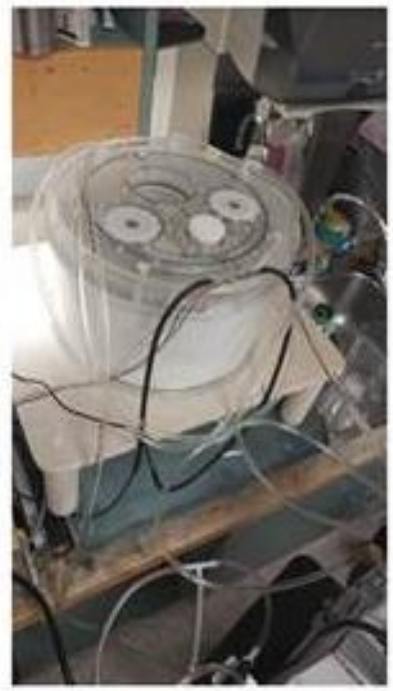

FIGURE 5-17 THREE LAYER OF LID ASSEMBLY AND WIRINGS.

After bringing the system to the equilibrium at the temperature about $4^{\circ} \mathrm{C}$, the water calorimeter was positioned under a calibrated Elekta. Synergy MLCi linear accelerator, and measurements were done under a $6 \mathrm{MV}$ beam at a source to surface (of Mylar) distance of $115[\mathrm{~cm}]$, with a field size of $5 \times 5\left[\mathrm{~cm}^{2}\right]$ and $400[\mathrm{MU} / 40 \mathrm{~s}]$ at the point of measurement. Seven measurements were performed, and the results from the seven runs were analyzed. 
(a)

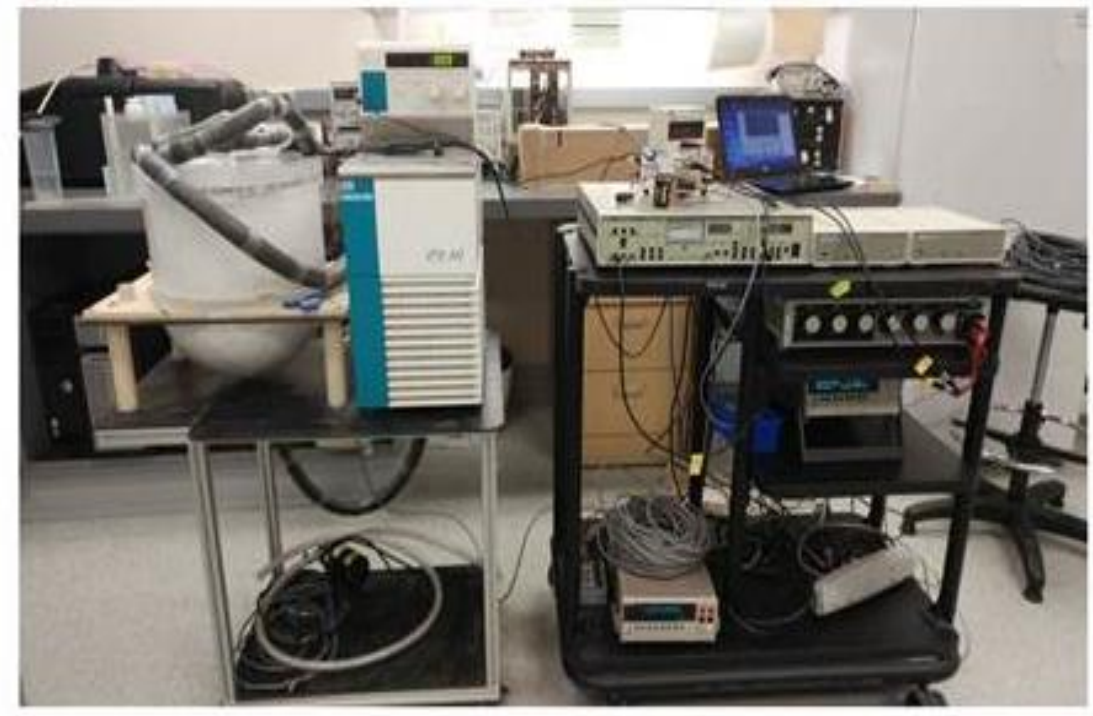

(b)

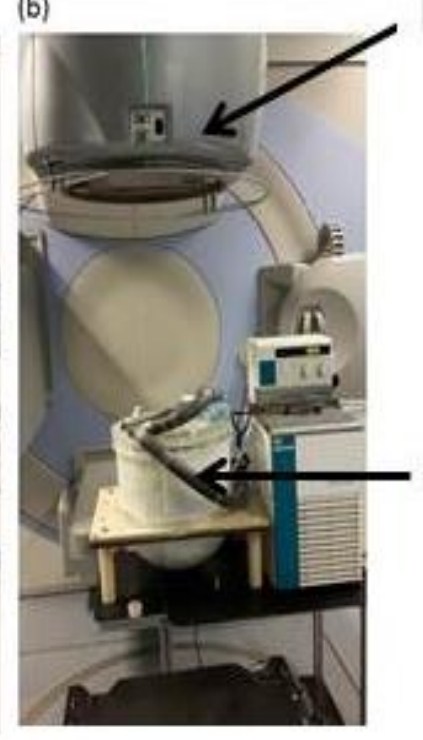

linear accelerator

Water Calorimeter

FIGURE 5-18 WATER CALORIMETER SET-UP (A) WITH ALL ELECTRONICS AND CIRCUITS (B) FOR APPLYING MEASUREMENTS UNDER LINEAR ACCELERATOR.

\subsection{MRI Imaging and Measurements}

Following the setup of the calorimeter, the device was imaged on a Philips $1.5[\mathrm{~T}] \mathrm{MRI}$ scanner. This was intended to demonstrate image quality when used in MRI-linac given MRI imaging is intended for use to position the thermistors in place. Both T1 and T2 Sequences were used for imaging, and three different slice thicknesses were used (see Table 5-7). As is always the case, the signal to noise ratio (SNR) improves at larger slice thicknesses at the expense of resolution. Given accurate positioning is the goal of this project, the intent was to see if at smallest slice thickness possible (1 [mm]), the SNR is large enough to be able to evaluate the tip of thermistors.

Figure 5-19 shows the setup inside the MRI, as well as the MRI images obtained from the sequences used.

The images are extremely encouraging as our device is, to our knowledge, the first calorimeter that can be MRI-imaged with minimal artifacts. The MRI imaging should allow for proper positioning of the calorimeter tank in the MRI-linac with an uncertainty of about 
1 [mm]. Given the absence of lasers and outside markers, calorimeter imaging can be an efficient way of positioning for accurate dosimetry.

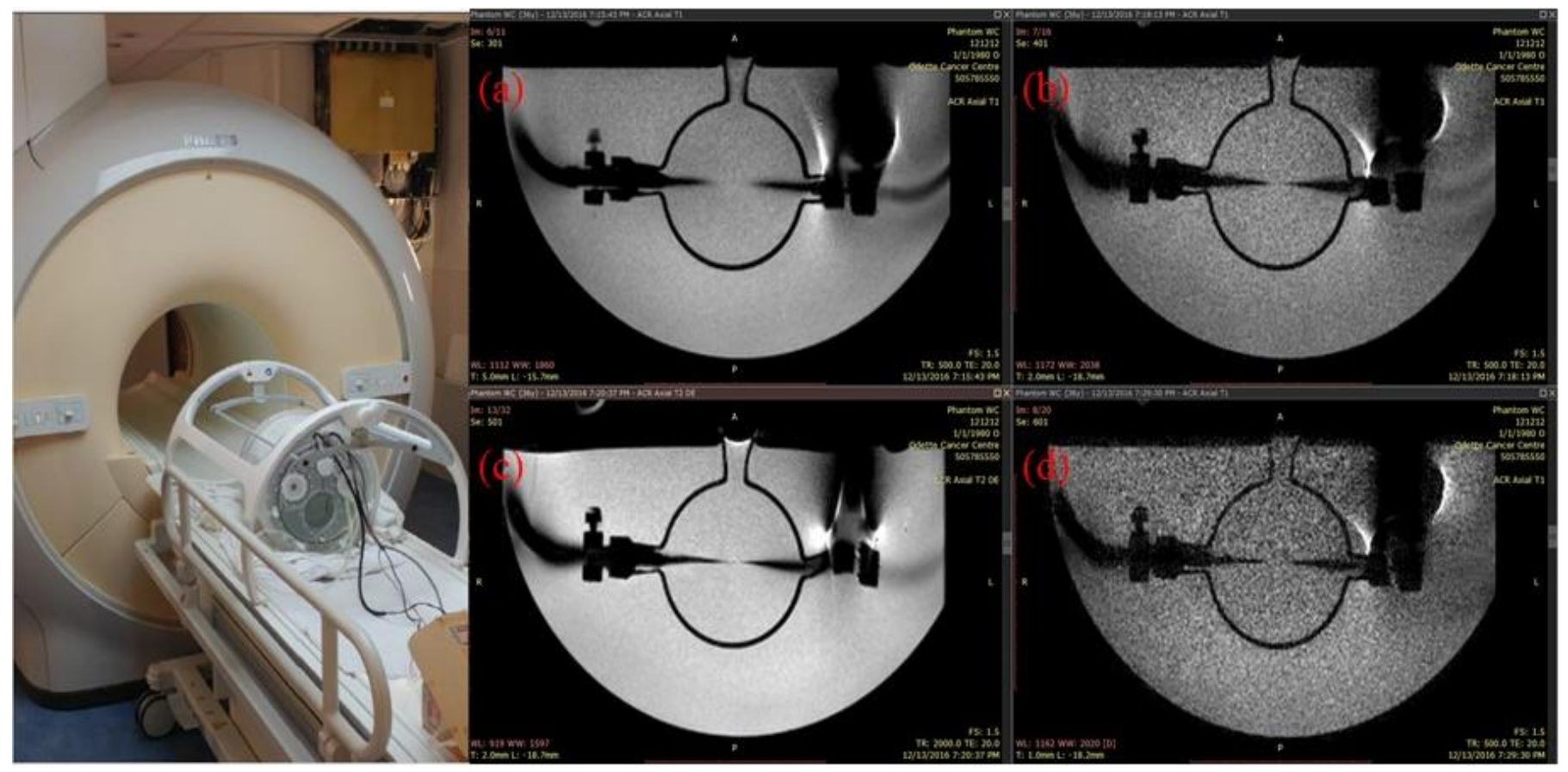

FIGURE 5-19 THE RIGHT IMAGE INDICATES THE POSITIONING OF THE WATER CALORIMETER INSIDE THE MRI. IMAGE IN THE LEFT INDICATES DIFFERENT SEQUENCES OF THE MRI IMAGES.

TABLE5-7 SEQUENCES AND SLICE THICKNESSES OF MRI-IMAGING

\begin{tabular}{|c|c|c|}
\hline Images & Sequences & Slice thickness \\
\hline $\mathrm{a}$ & $\mathrm{T} 1 \mathrm{~W}$ & $5[\mathrm{~mm}]$ \\
\hline $\mathrm{b}$ & $\mathrm{T} 1 \mathrm{~W}$ & $2[\mathrm{~mm}]$ \\
\hline $\mathrm{c}$ & $\mathrm{T} 2 \mathrm{~W}$ & $2[\mathrm{~mm}]$ \\
\hline $\mathrm{d}$ & $\mathrm{T} 1 \mathrm{~W}$ & $1[\mathrm{~mm}]$ \\
\hline
\end{tabular}

\subsection{Corrections and Conversions}

As described in section 3.5, the dose to water was obtained by multiplying the corrected temperature measurement by the specific heat capacity of water. The corrected temperature is simply the measured radiation-induced temperature rise corrected for heat 
transfer and radiation perturbation effects (due to presence of non-water materials inside the calorimeter in the direct path of the radiation beam). The heat transfer correction due to conduction was simulated using COMSOL Multiphysics ${ }^{\mathrm{TM}}$ based on the Finite Element Method modelling (section 3.6), and the perturbation was calculated by using MonteCarlo simulations.

I. Heat transfer correction factor- This correction was calculated by approximately modelling the water calorimeter tank in an axially symmetric geometry in COMSOL Multiphysics $^{\mathrm{TM}}$. Only the heat transfer due to conduction was taken into account. The system was assumed to start from a perfect $4^{\circ} \mathrm{C}$ thermal equilibrium initial conditions. It should be noted that normally this is an accurate assumption, although in our specific preliminary set of measurements, due to some technical difficulties and time constraints, the tank was unable to reach perfect thermal equilibrium while we conducted our measurements. The radiation beam was modeled accurately by applying a twodimensional radiation-induced temperature gradient (based on the profile and percentage depth dose of our clinical beam) as heat source. The irradiation periods with the rest periods in between were also modelled accurately. Details of simulation were in general identical to those explained in section 3.5).

The temperature rise at the point of measurement was obtained and the ratio of the ideal to actual temperature rise comprised $k_{\mathrm{ht}}$. The $k_{\mathrm{ht}}$ results for the seven consecutives 40 [s], simulated irradiation runs modelled (as per our measurements) were $0.9905 \pm 0.0005$, $0.9898 \pm 0.0005, \quad 0.9894 \pm 0.0005, \quad 0.9888 \pm 0.0005, \quad 0.9885 \pm 0.0005, \quad 0.9896 \pm 0.0005$, $0.9896 \pm 0.0005,0.9895 \pm 0.0005$.

II. Perturbation correction factor- The product of the radiation field perturbation occurs due to the effects of non-water materials present in the water calorimeter. $k_{\mathrm{p}}$, is the ratio of the measured dose at the thermistors for two similar Monte Carlo simulations, one in pure water and one with non-water materials modelled, for e.g. the glass vessel. 
Two opposing effects (attenuation and scatter) defines the magnitude of the perturbation ratio: A deduction of the dose from attenuation requires a correction factor higher than unity, and an increase in dose due to scatter, will result in a correction factor less than unity.

In this work, GEANT4 Monte Carlo (MC) simulation of the geometrical setup was used to determine the value of $k_{\mathrm{p}}$ correction and it was determined to be $1.004 \pm 0.594 \%$. This value is specific to the particular experimental setup used in our absorbed dose measurements (6 MV photon spectrum, $115 \mathrm{SSD}, 5 \times 5$ [cm²] field size).

\subsection{Absorbed Dose Measurements}

In this section, a summary of the results of the absorbed dose measurements performed using the in-house built water calorimeter are presented. The raw monitor unit-normalized dose results from the 7 calorimetric runs, along with the calculated corrections (Section 5.9), and the final corrected normalized dose results are shown in Table 5-8. The raw measured results are determined by multiplying the measured temperature rise per each run (using the extrapolation to the mid-run technique described in Section 3.2 and section $3.5)$, by the water's specific heat capacity. Note that the temperature rise itself is determined by relating the out-of-balance bridge voltage reading (the measured) to the thermistor temperature-induced resistance change (Ohm Calibration, Section 5.6.3), which itself is directly related to the actual temperature change at the point of the thermistors (Thermistor calibration, Section 5.6.2).

As shown in Table 5-8, the absorbed dose to water normalized to MU delivered, under the measurement conditions described $(6.76$ [cm] depth, SSD of $115[\mathrm{~cm}]$, field size of $5 \times 5$ [cm²], $6 \mathrm{MV}$ Elekta beam) was 0.534 [cGy/MU]. This value was $4.2 \%$ different from our hand calculated expected result. The large uncertainty (and variation) of the measurements as seen in Table 5-8 is due to lack of complete thermal equilibrium during the measurements. Indeed, given time constraints and the initial challenges faced with the operation of the calorimeter, we did observe larger than expected drifts that resulted in larger than expected standard deviation of the signals. Further work towards perfecting 
the design (specifically the stirrers) based on the initial set of measurements is currently underway.

TABLE5-8 SUMMARY OF DOSE MEASUREMENTS PERFORMED USING THE WATER CALORIMETER FOR 400 MU/40S DELIVERIES UNDER THE MEASUREMENT CONDITIONS.

\begin{tabular}{|c|c|c|c|c|c|}
\hline $\begin{array}{c}\text { Number of } \\
\text { runs }\end{array}$ & $\begin{array}{c}\text { Signal } \\
\text { [cGy/MU] }\end{array}$ & $\boldsymbol{k}_{\mathrm{ht}}$ & $\boldsymbol{k}_{\mathbf{h d}}$ & $\boldsymbol{k}_{\mathbf{p}}$ & $\begin{array}{c}\text { Dose } \\
\text { [cGy/MU] }\end{array}$ \\
\hline 1 & 0.557 & 0.9905 & 1 & 1.004 & 0.554 \\
\hline 2 & 0.542 & 0.9898 & 1 & 1.004 & 0.538 \\
\hline 3 & 0.537 & 0.9894 & 1 & 1.004 & 0.533 \\
\hline 4 & 0.535 & 0.9888 & 1 & 1.004 & 0.531 \\
\hline 5 & 0.553 & 0.9885 & 1 & 1.004 & 0.548 \\
\hline 6 & 0.509 & 0.9896 & 1 & 1.004 & 0.505 \\
\hline 7 & 0.553 & 0.9896 & 1 & 1.004 & 0.549 \\
\hline Average & 0.537 & 0.9895 & 1 & 1.004 & 0.533 \\
\hline \%STDEV & $3.5 \%$ & & 1 & & $3.5 \%$ \\
\hline Expected & & 0.9896 & & & $\mathbf{0 . 5 1 1}$ \\
\hline
\end{tabular}




\section{CHAPTER 6 - SUMMARY AND CONCLUSIONS}

The aim of this work has been to design, simulate, and develop a portable and MRI compatible water calorimeter with the eventual goal of showing feasibility of performing water calorimetry under MRI-linac and Gamma Knife ${ }^{\circledR}$ radiation therapy units. Water calorimeters determine radiation energy absorbed in a sensitive volume by measuring radiation-induced temperature rise of the volume (related by the medium specific heat capacity). In this formalism, one important correction factor is heat transfer correction $k_{\mathrm{ht}}$. It compensates for heat gain/loss due to conductive and convective effects, and is numerically calculated as ratio of temperature rise in the absence of heat loss to that in the presence of heat loss. A $4^{\circ} \mathrm{C}$ stagnant water calorimeter ensures convection is minimal. Water calorimetry at Primary standard laboratories is well established for high energy photon beams, and the reference dosimetry for various modalities in clinics to date have been performed by using the calibration results from high energy photon beams and applying measured or calculated correction factors to correct for differences between calibration conditions and measurement conditions. However, with the advent of highly specialized clinical accelerators such as Cyber Knife ${ }^{\circledR}$ and Tomotherapy ${ }^{\circledR}$, or the integration of high field MRI with modern linear accelerators, or even Gamma Knife $®$, reference dosimetry is becoming ever more challenging.

In this work, we went through a set of numerical optimizations to better understand the behavior of calorimeter system with a focus on MRI-linac applications. We used our experience and simulation results to design and build a water calorimeter that is vastly different in shape, form and size from previously accepted designs. Our calorimeter has the unique advantage of being fully MRI-safe and can be imaged using MRI and CBCT, and can be used in MRI-linac and Gamma Knife ${ }^{\circledR}$. Indeed, the internal structures can be imaged with MRI for positioning purposes. The calorimeter can be irradiated from the top in MRL and conventional radiotherapy units, while when used horizontally, our design will allow for measurement of absolute dose in Gamma Knife ${ }^{\circledR}$ with 192 Co-60 beams incident through the hemispherical end (resulting in equal path-lengths to point of measurement). This work leads the way towards establishment of absolute dosimetry 
protocols based on water calorimetry in these specialized radiotherapy delivery equipment.

A commercial finite element software was used to evaluate several water calorimeter designs with different insulation materials, thicknesses. Channels allowing coolant to travel around water calorimeter (to sustain water calorimeter at $4^{\circ} \mathrm{C}$ ) were modeled, and worst-case scenario variations in the temperature of the coolant was simulated for optimization purposes. Additionally, several calorimeter vessel design parameters (front/back glass thickness/separation, diameter) were also simulated and optimized. Optimization is based on evaluating long term calorimeter drift as well as variations in and magnitude of $k_{\mathrm{ht}}$. The final selected water calorimeter design achieved a modest drift of (4.27E-07 [K/s]) after 24 [h] for the worst-case coolant temperature variation. This design consists of coolant channels being encompassed on both sides by Cryogel $\circledast$ insulation. For the simulations with the high energy photon beams incident from the top, glass thickness plays the largest effect on $k_{\mathrm{ht}}$ with variation of up to $0.6 \%$ in the first run for thicknesses ranging between $0.5-2.3 \mathrm{~mm}$. Subsequent runs vary only within $0.1 \%$ with glass thickness. Other factors such as vessel radius and top/bottom glass separation have sub $0.1 \%$ effects on $k_{\mathrm{ht}}$ (figure $5-6$ ). Based on the results of the most optimal calorimeter as well as minor adjustments based on experience, a water calorimeter was constructed in house in the Sunnybrook Odette Cancer Center machine shop. The dimensions and design selected ensured thermal stability while ensuring the calorimeter fits inside the bore of an MRI-Linac and Gamma Knife ${ }^{B}$ collimator ring. A set of preliminary measurements under a conventional $6 \mathrm{MV}$ photon beam from an Elekta Synergy unit was performed. Further modifications based on initial experience with the unit are underway. Future measurements in conventional linacs, MRI-linac, and Gamma Knife ${ }^{\circledR}$ will be performed to evaluate the performance of our calorimeter in these novel modalities. A spherical vessel design for use in Gamma Knife ${ }^{\circledR}$ is also underway. 


\section{REFERENCES LIST}

[1] Canadian cancer statistics 2016. $1^{\text {st }}$ ed. Toronto, Ont.: Canadian Cancer Society, 2016.

[2] National Cancer Institute, Radiation Therapy for Cancer: Questions and Answers. 2009.

[3] Wondergem, J., "Molecular and Cellular Radiobiology", in Radiation Biology: A Handbook for Teachers and Students, Training Course Series 42. (International Atomic Energy Agency, Vienna, Austria, 2010).

[4] Podgorsak, E. B., "Treatment Machines for External Beam Radiotherapy", in Review of Radiation Oncology Physics: A Handbook for Teachers and Students, edited by E. B. Podgorsak (International Atomic Energy Agency, Vienna, Austria, 2005).

[5] International Commission of Radiation Units \& Measurements (1976) Report 24 "Determination of absorbed dose in a patient irradiated by beams of $X$ or gamma rays in radiotherapy procedures".

[6] Van Dyk, J., "The Modern Technology of Radiation Oncology: A Compendium for Medical Physicists and Radiation Oncologists", edited by J. Van Dyk (Medical Physics Publishing, Madison, USA,1999)

[7] Almond, P., Biggs, P., Coursey, B., et al., AAPM's TG-51 protocol for clinical reference dosimetry of high-energy photon and electron beams, Med Phys, 26, 1847-1870, (1999).

[8] Siebers, J., \& Hugo, G., "Basic Radiation Interactions, Definition of Dosimetric Quantities, and Data Sources", in Clinical Dosimetry Measurements in Radiotherapy, edited by D. W. Rogers and J. E. Cygler (Medical Physics Publishing, Madison, USA, 2009).

[9] Khan, F. M. The Physics of Radiation Therapy, ed. 3, pp. 164 (Lippincott Williams \& Wilkins, Philadelphia, USA, 2003).

[10] J. Seuntjens and M. McEwen, "The calibration chain: Role of BIPM, PSDLs and ADCLs," in Clinical Dosimetry Measurements in Radiotherapy, edited by D. W. Rogers and J. E. Cygler (Medical Physics Publishing, Madison, 2009).

[11] P. J. Allisy, D. T. Burns, and P. Andreo, "International framework of traceability for radiation dosimetry quantities," Metrologia 46, S1-S8 (2009).

[12] J. Seuntjens and S. Duane, "Photon absorbed dose standards," Metrologia 46, S39-S58 (2009). 
[13] Sarfehnia, A., Ph.D. dissertation, McGill University, 2010.

[14] Carter, A. H., "Classical and Statistical Thermodynamics", (Prentice Hall, Upper Saddle River, USA, 2001).

[15] C. K. Ross and N. V. Klassen, "Water calorimetry for radiation dosimetry," Phys. Med. Biol. 41, 1-29 (1996).

[16] Strang, G., \& Fix, G., "An Analysis of the Finite Element Method", Prentice Hall Series in Automatic Computation, (Prentice Hall, Upper Saddle River, USA, 1973).

[17] Renaud, J., Sarfehnia, A., Marchant, K., McEwen, M., Ross, C., \& Seuntjens, J. (2015). Direct measurement of electron beam quality conversion factors using water calorimetry. Medical physics, 42(11),6357-6368.

[18] Sarfehnia, A., Kawrakow, I., \& Seuntjens, J. (2010). Direct measurement of absorbed dose to water in HDR 1192r brachytherapy: Water calorimetry, ionization chamber, Gafchromic film, and TG-43. Medical physics, 37(4), 1924-1932.

[19] Sarfehnia, A., Clasie, B., Chung, E., Lu, H. M., Flanz, J.,Cascio, E., \& Seuntjens, J. (2010). Direct absorbed dose to water determination based on water calorimetry in scanning proton beam delivery. Medical physics, 37(7), 3541-3550. 